\title{
ZUR DECLINATION.
}

\section{GERMANISGH $A$ E O IN IDEN FNIUNGEN IOES NOMENS UNI IUE ENTSTEHUNG I)ES $O\left(a_{2}\right)$.}

Meine s. 161 des vorigen aufsatzes gemachte bemerkung. dass wir kiinftig $\bar{a}$ und $\bar{o}$ werden zu scheiden haben, veranlasst mich an dieser stelle in möglichster kïrze meine ansicht iber das selicksal der friiheren $\bar{a} \bar{c} \bar{o}$ im germanischen darzulegen, wohei ich besonders die endsilben des nomens ins auge $\mathrm{zu}$ fassen gedenke.

Das $\bar{a}$ der grundsprache, griech. lat. $\bar{a}$ lett. $\bar{a}$ lit. $\grave{o}$, war noch ursprünglich germanisch $\bar{a}$, das $\bar{o}$ der grundsprache, griech. lat. $\bar{o}$ lit. lett. $\bar{n}$, war germ. $\bar{\sigma}$. Jenes $\bar{a}$ war, wie schon Kuhns zs. XXIV, 508 bemerkt, ein $\bar{a}$ als die gallisehen wörter $b r \bar{a} c a$, Dannuvius ins germanische ühergiengen. Statt germ. $\bar{a}$ und $\bar{o}$ ist nicht mit Sievers, Beitr. V. 133 offines und geschlossenes is (Sicvers $\hat{o}^{2}$ und $\left.\hat{o}^{1}\right) z u$ setzen, wenigstens nicht für das gemeingermanische. Denn dieses germ. $\bar{o}$ aus urspr. $\bar{o}$ war ein langes offines $\bar{\pi}, 1)$ weil es im oberdeutseben und fränkischen zu uo diphthongiert wird, ahol. tuon, chuo, fuoz, bruoch palus, fiotur scheide, und nur offnes $\bar{o}$ diese diphthongierung erfährt (s. Kuhns zs. aao. $509 \mathrm{f}$.), und 2) weil es dem germ. $\bar{c}$ aus altem $\bar{c},=$ griech. lat. $\bar{e}$ slav. $\bar{e}(\grave{e})$ lett. lit. $\dot{e}$, parallel war, das ein offnes $\bar{e}$ gewesen sein muss, weil es im nordischen und westgermanischen zu $\bar{a}$ geworden ist (woraus secundär wieder engl.-fries. $\hat{a}, \hat{e})$. Jenes germ. $\ddot{a}$ fiel später, ausser in endsilben, mit germ. " zusammen, indem es gemeingermanisch " ward.

Die urspriungliche versehiedenheit von germ. $\bar{a}$ und $\bar{o}$ ist auch fitr die stammsilben noch nachweishar. Nur vor urgerm. $o, \bar{o}$ und $u, \bar{u}$, nicht vor urgerm. $a, \bar{a}$ schwindet ein an an- 
lautendem $k$-laut baftendes mitlautendes $u$ (doch nicht anlautendes urspr. $v$, auf welches Bezzenberger in seinen Beitr. V. $175 \mathrm{f}$. die regel ausdehnt, und ebenfalls nicht ein anlautendes $v$, das ein vorhergehendes $g$ oder urspr. $g h$ in germanischen wie im lateinischen frühe verlor, got. varms): das $u$ ist geschwunden wie in ahd. houwan (= slav. kova, vgl. lat. $c \bar{u}-d o)$ so in as. kô ahd. chuo, aber geblieben wie in hvass so in ae. hwôsta husten und dem dazu gehörigen starken verb mit dem präsens auf - jo-, ae. hwêsan, dessen vocal ursprünglich $\bar{a}$ war nach lett. kāsit lit. kisseti husten. (Hiernach ist Kluge, Conjug. s. $4.1 \mathrm{zu}$ berichtigen: got. hafja = lat. capio muss urspriinglich palatales $k$ haben und ist bei Kiuge $z u$ streichen, es bat nichts zu tun mit armen. kapel capere, dessen anlaut urspr. gu, vgl. Bezzenberger a. a. o. 175 unten).

Uebereinstimmend in allen germanischen dialecten ist das alte $\bar{a}$ überhaupt nur in betonter stammsilbe zu $\bar{o}$ geworden. In unbetonter silbe im inlaut set\%t das englisch-friesische und das niederdeutsche in weit geringerer ausdehnung den übergang des $\bar{a} \mathrm{zu} \bar{o}$ voraus, als wir denselben im bochdeutschen und gotischen vollzogen sehen. Im gemeinwestgermanischen auslaut, $d . h$. in germanischer auf vocal, nasalierten vocal oder vocal $+z$ ausgehender endsilbe, germ. $-\bar{a},-\bar{a}^{n}$ und $-\bar{a} z$, ist das $\bar{a}$ in keinem westgermanischen dialect, auch nicht im hochdeutschen, zu $\bar{o}$ geworden. im auslaut werden also germ. $a$ und $\bar{\sigma}$ in allen westgernanischen dialecten scharf geschieden. Dafiir ist jedoch in germanischer endsilbe in falle der nasalicrung und vor auslautendem $z$ rerm. $\bar{\pi}$ in westgermanischen mit gern. $\bar{i}$ zusammengefallen, germ. $-\bar{\pi} n,-\bar{a} z$ mit germ. $-\bar{p} n,-\bar{p} z$, da grerm. i in allen fällen, ausser im reinen auslaut, gemein-

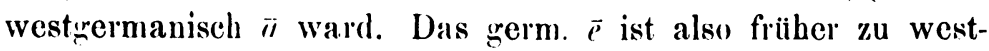
grerni. $\bar{a}$ geworden, als das germ. $\bar{a} \% u \overline{~(n a t u ̈ l i c h ~ e n t s t a n d ~}$ aus dem $\bar{e}$-laut der denkbar offenste und reinste $\bar{a}$-laut, der des dänischen $a$ in have, ein $a$-laut, der wie wir es am dänischen sehen, in beständiger gefahr ist wider zum $\ddot{i}$-laut $z u$ werden, der laher auch im englischen und friesischen wider zu $\hat{a}$. dann $\hat{e}$, geworden ist, während dagegen das germanische $\bar{a}$, das zu $\bar{o}$ ward, etwa unser nhd. $i$ in hahen, oder auch noch dunkler war). Denn das $\bar{c}$ ward westgerm. $\bar{n}$ iberall ausser im auslaut, aber zu cincr zeit wo es in den endsilben gern. 
$-\bar{e}^{n}$ und $-\bar{e} z$ noch im inlaut, nur in germ. $-\bar{e}$ (urspr. $-\bar{e}$ und $-\bar{e} t$ ) im auslaut stand, ebenso ward das germ. $\bar{a}$ im hochdeutschen zu $\bar{o}$ therall ausser im auslaut, aber zu einer zeit, in welcher durch abfall des aus $z$ entstandenen $r$ und aufgebung der nasalierung das $\bar{a}$ der endsilben germ. $-\bar{a}^{n}$ und $-\bar{a} z$ im westgermanischen in den auslaut gerlickt war.

Das auslautende ältere westgermanische $-\bar{a}$ und $-\bar{o}$ wird in unbetonter silbe gemeinwestgermanisch $\mathrm{zu}-a$ und $-o$ verkilrzt. Im englisch-friesischen verfällt dann jenes - $a$ der verdünnung des $a$ zu $\ddot{a}(e)$, dieses -o wird englisch-friesisch - $a$ (Sweet, Transactions 1875- 6 , s. 543 ff., Paul, Beitr. IV, 342 -346).

Das geschick der germ. auslautenden $-\bar{a}-\bar{e}-\bar{n},-\bar{a} n-\bar{e} n-\bar{n} n$ und $-\bar{a} z-\bar{c} z-\bar{o} z$ zeigt folgende tabelle.

Auslautendes in unbetonter silbe wird germ. $-\vec{o}$

$$
\begin{aligned}
& "-\bar{o}^{n},-\bar{a} z \text { ahd. as. }-\sigma \text { afr. ae. }-a=\text { got. }-\hat{o},-\hat{s} \text { an. }-a,-a r \text {. } \\
& -\quad-\bar{a} \\
& \eta \quad-\bar{a}^{n},-\bar{a} z \\
& \geqslant-\bar{e} \\
& \text {, - } \bar{e}^{n},-\bar{c} z \\
& \text { ahd. } \left.-u \text { as. afr. ae. }-u,--^{1}\right)=\text { got. }-a \text { an. }-u
\end{aligned}
$$

Dass die westgermanische doppelheit des auslauts, -o $(-a)$ und $-a(-e)$, auf eine vorgermanische doppelheit $\bar{o}$ und $\bar{a}$ zurtuckgehe, vermutete schon Paul, Beitr. IV, $348 \mathrm{f}$., $356 \mathrm{f}$. Es stellten sich ihm aber bei der zurickftibrung der westgermanischen auslaute auf diesen alten unterschied scbwierigkeiten in den weg, und er kam $\mathbf{s .} 357 \mathrm{zu}$ dem resultate, dass sich die annahme einer urgermanischen scheidung der beiden längen 'nicht stricte zurlickweisen, aher auch nicht hinlänglich motivieren' lasse, und dass wir genötigt bleiben 'als bedingung fur die westgermanische scheidung von $a$ und $o$ immer noch andere, teils vermutbare, teils verborgene und vielleicht rein zufällige

1) Das aus $\bar{\sigma}$ entstandene $\boldsymbol{u}$ schwindet im englischen nach langer silbe, im fries. und nd. nach langer und mehreren silben, dagegen im hochdeutschen schwindet dieses - $u$ nicht, der plur. neutr. ahd. joch, wort ist der eingedrungene plural der consonantischen declination $-a$, wie in lat. juga griech. క̌vó. (Ebenso fasst die sache auf (r. Mahlow, Die langen vocale A E 0 , s. 77 unten.)

2) Dass germ. - $\bar{a}^{n}$ got. - $\hat{\delta}$ wird ist nicht völlig unanfechtbar, denn die annahme, dass es got. - $a$ werde, acc. gib $\lambda$, ist nicht in zwingender weise $\mathrm{zu}$ widerlegen. 
momente anzunehmen'. Beitr. VI, 184 gielt Paul jene rermutung vollständig auf: er erklärt dass die urspr. $\bar{a}$ und $\bar{o}$ wahrscheinlich im urgermanischen eben so vollständig zusammengefallen sind, wie die entsprechenden kürzen, und er hïlt es jetzt furr viel wahrscheinlicher, dass die westgerm. spaltung auf verschiedener tonintensität beruhe.

Sievers, Beitr. V, 133 vermutet ebenfalls, dass die spaltung in gekürztes $a$ einer- und $o, u$ andererseits auf zwei im urgermanischen verschiedene laute zurückgehe, die er als offenes und geschlossenes $\hat{o}$ ansetzt, da es ihm als ausgemacht gilt, dass ursprüngliches $\hat{a}$ auch am wortende sich germ. zu $\hat{o}$ umgestaltete. In den Morphol. unters. I, 142 stellt Sievers fur einen andern ort eine besprechung der gesetze in aussicht, nach welchen ein durch einen consonanten (ausser $-t$ ?) gedecktes urspr. $-\bar{a}-[$ d. h. jetzt $\bar{a} \bar{e} \overline{0}]$ im ahd. as. entweder als $-a$ oder als -0 , im ags. als -e resp. $-a$ erscheint: ursprünglich auslautendes $-\bar{a}$ [wie eben?] wird ihm in allen germ. sprachen mit ausnahme des gotischen stets zu $u$.

Osthoff, Morph. unters. I, s. $240 \mathrm{ff}$. führt alle ahd. $-a$, ae. -e auf urgerm. $\bar{e}$ zurlick. Wo sich ein urspr. $\bar{e}$ nicht bietet, sondern statt dessen ein $\bar{a}$ oder $\bar{o}$, ruft Osthoff ein $j$ zu halfe, das ein folgendes nasaliertes $\bar{o}$, auch ein aus $\bar{a}$ entstandenes, (oder nach der dem Osthoffschen gesetze von Paul, Beitr. VI, $209 \mathrm{f}$. gegebenen allgemeineren fassung, ein jedes, auch nicht nasaliertes $\bar{o}$ ) in $\bar{e}$ gewandelt habe.

Die zuruckfuhrung der westgermanischen auslaute auf die von der grundsprache her rerschiedeuen längen in der von mir angegebenen weise:

gemeinwestgerm. $-a,-e$ auf urspr. $\vec{a}$ oder $\bar{e}$,

lïsst sich ohne übergrosse schwierigkeit durehfuhren.

Es ist nicht richtig, dass jeder urspriinglich auslautende $\bar{a}$-laut nach der alten bezeichnung, also, um von $\bar{e}$ nicht zu reden, dass urspr. $-\bar{a}$ und $-\bar{o}$ in gleicher weise in allen germanischen dialecten mit ausnahme des gotischen $-u$ worde. Dass $-\bar{a}$ westgerm. $-a$ bleibt, lehrt das den accusativ sing. der pronominalen declination angehängte $-\bar{a}$, as. thena, ae. Jone $=$ got. pana, im adj. as. hêlagna ae. $-n e=$ got. -ana: dass dies $-a$ nicht cin altes $-\bar{e}$ sondern ein altes $-\bar{u}$ ist, zeigt got. 
huanô-h, hvarjanô-h.1) Altes $-\bar{\iota}$ hat ahd. as. fora ae. fore $=$ got. faura, skr. pura $\bar{a}^{\prime}$. Dass $-\bar{o}$ ausser im gotischen $-u$ wird, zeigt der nom. acc. plur. der neutra auf urspr. -o-, und ebenso die damit identische endung des nom. ace. dual mase. (got. $p \hat{o}$ $=$ gr. $\tau(\dot{v}$, slav. $t \bar{a}$, lit. $t i-d u)$, die vom sprachgefühl als neutr. plur. gefasst, die syntactische regel aufkommen liess, 'masc. + fem. wird durch den plur. neutr. gegeben'.2) Die regel ist also 'auslautendes germ. $-\bar{o}$ wird westgerm. $-u$, germ. $-\bar{a}$ wird westgerm. $-a$ '.

Diese einfache regel vermochte nicht aufzukommen, so lange es keinem in den sinn kommen konnte etwas anderes, als $-\bar{u}$ als ursprüngliche endung des nom. sing. fem. der $\bar{a}$-declination anzunelımen. Dies war iberhaupt der punkt, der der erkenntnis hindernd im wege stand, dass germ. $\bar{a}$ und $\bar{o}$ in endsilben nicht zusammengefallen sind. Nach dem aber, was wir von F. de Saussure, Syst. prin. 138, gelernt haben, dass sich $\bar{a}$ zu $\bar{o}$ verhält wie $e+$ cons. zu $a+$ cons., müste für den nom. sing. als starken casus die endung urspr. $-\bar{o}$ erschlossen werden, selbst wenn dies $-\bar{o}$ in keiner sprache erhalten wäre. Das $-\bar{a}$ - und $-\bar{o}$ verhalten sich $z u$ einander wie das -es- und

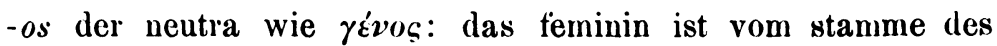
masculins gebildet durch den consonanten $A$ (s. u.), das $-\bar{a}$ - des feminins ist entstanden aus $-e A-$, das $-\bar{o}$ aus $-O A$. Ich habe aus diesem grunde schon vor einem jahre Engl. stud. III. 150 dem nom. sing. fem. die endung - $\bar{o}$ beigelegt. Das gesetz, nach wolchem im nom. sing. der $o$-laut eintreten muste, wird unten dargelegt werden in dem abschnitt aber die entstehung des 0 . Auf das $-\bar{o}$ weist ausser dem germanischen mit notwendigkeit das umbrisch-oskische zurtick: die endung des nom. sing. fem. und ebenso des nom. ace. plur. neutr. ist umbr. osk. -o (in

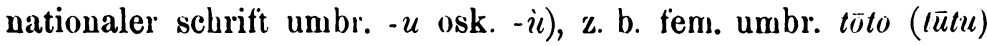

1) Bei Sievers formulierung des gesetzes wuss die angehängte partikel fiir ein altes - $i m$ erkliirt werden: die consequenz wäre die lehre, dass urspr. - $-i m$, germ. - $a^{n}$ auslautend unbetont got. - $a$ werde, dass also got. giba der regelrechte accusativ sei (so Mahlow, Die langen vocale s. $56 \mathrm{fti}$., (it fii.). Lin solches got. $-a$ aus $-\bar{a}^{n}$ miiste lang sein, pan $\hat{a}$, gibâ.

2) Beispiel altfries. Luss. alder eltern (aus germ. alfpizo, dual von consonantischem stamme auf $-i z$ - gebildet mit dem dem masc. der $v$-decl. entnommenen suffix), zu dem später noch ein neutraler sing. geschaffen ward. 
osk. tovto (rimbii), wie neutr. pl. osk. comono, das - o kamn unmöglich aus langem oder kur\%em - $a$ hervorgeyangen sein. Das -a der ïstlichen sprachen, indisch, iranisch. slavisch, kann so gut $-\bar{\prime}$ wie $-\bar{u}$ sein: dass, wo wie $\%$ b. im griechischen sicher $-\bar{a}$ vorliegt, das - $a$ - der schwachen casus in den nom. gedrungen ist, kann nicht im mindesten wunder nehmen. $\left.{ }^{1}\right)$

Der ace. sing. fem. hat bistorisch überall die endung -äm, osk. - $\bar{a} m$ slav. $-a$ etc., germ. $-\bar{a}^{n}$, woraus nach unserer regel ahd. as. - $a$ afi. ae. - e. Osthoff erklärt Morph. us. I. 267 ff. dies ahd. $-a$ ae. $-e$ aus $-\bar{e} n$, das von den $j \bar{a}$-stämmen her verallgemeinert sei. Aber dass urspr. $j \bar{u}$ zu grerm. $j \bar{r}$ wird, bestreite ich (wenn iberhaupt, daun wird bloss $j \bar{o} z u$ jë), ausserdem ist unser gemeinwestgerm. - $a$ des accusativs regelrechte kürzung des langen $-\bar{a}$ aus gern. $-\bar{a}^{n}$ urspr. $-\bar{a} m$ im acc. des pronomens, afi. ae. an. thâa, $\partial \hat{a}$. - Die älteste endung des acc. fem. wird nicht $-\bar{a} m$, dessen $\bar{a}$ aus den schwachen casus eingedrungen ist, sondern $-\bar{o} m$ gewesen sein. Dieses $-\bar{o} m$, germ. $-\bar{o} n$ haben die adverbien auf got. - $\hat{o}$ ahd. as. $-o$ an. $-a$ wie got. galeik $\hat{o}$ ahd. gilicho an. lika erhalten, die Osthoff, Kuhns zs. XXIII, $90 \mathrm{ti}$., Morph. us. I $271 \mathrm{f}$. als accusative des fenı. erklärt hat. Im friesisch-englischen haben diese adverbien die wandlung des casus in die schwache form nitgemacht, afr. ae. $-e$, wie die adverbien auf griech. $-\eta p$, lat. -am. - Das ae. zifu, gen. dat. acc. zife, ist germ. nom. $-\bar{i}$ gen. $-\bar{a} z$ loc. $-a i$ acc. $-\bar{a}^{n}$. Das germ. $-\bar{a} z$ des genitivs ist völlig regelrecht ahd. as. $-a$ afr. ae. -e - an. -ar got. òs in der nominalen wie in der pronominalen

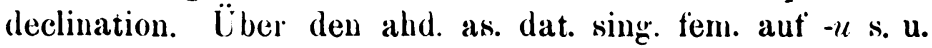

Den casus des singulars, nom. $-\overline{0}$ acc. $-\bar{u}^{\prime \prime}$, entsprechend lauteten dic easus des plurals fem. germ. nom. -izz ace. -ianz. Das $-o z$ des nom. plur. (mit dem $\partial$ des starken casus) wird

1) 1)as germ. $a$, das in der tonsilbe gemeingerm. $o$, gotisch geschlossenes i wird, wird vom slavischen aus einem östlichen dialecte mit dem vocialismus des gotischeu als * $i$ aufgenommen, das im slavischen diphthongierung zu $o u$, woralls endlich $\tilde{u}$, erfihit: slav. plug $\breve{u}$, Inuãut, kupiajelt (aus germ. Kaupajedi, got. liaupip, Mahlow s. 151 ohen) (ebenso wird schwed. $i$ im altruss. $u$, s. Thomsen, Urspr. d. russ. staites s. 76). In demselben östlichen dialecte scheint auslautendes germ. -o zu " geworden zu sein (wic vielleicht im westgermanischen und nordischen): diss slav. bulig buche setzt ein *bikiu (aus germ. bako) voraus. 
alıd. -0 ae. $-a$ : dieses ae. $-a$ ist die regelmässige endung, jenes ahd. -o sehen wir in alemann. kebo, sunto und im plural fem. der pronominalen declination dio, drio, plinto, ungekïrzt in ahd. $z w u o=$ as. $t w \hat{o}$. Fur dieses ahd. $-o$ in plur. fem. der pronominalen declination ist die anuabme einer formubertragung aus dem masc. ausgeschlossen. Will man es aus einem aus urspr. $-\bar{a} s$ hervorgegangenen gemeingerm. $-\bar{o} z$ erklären, dann muss man um die germ. entsprechung des $-\bar{a} s$ des gen. sing. zu erklären zu künstlichen mitteln greifen. Das $\bar{a} \boldsymbol{n}_{z}$ des acc. plur. wird

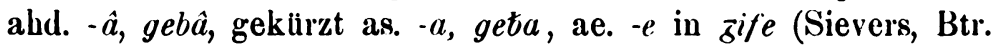
I. 487). Die läng.e des auslautenden vocals in ahd. gebâ ist etwas eben so singuläres wie der fall eines germanischen nasalierten langen vocals vor auslautendem $z \cdot-\bar{a}^{n} z$ ist schon von Mahlow, Die langen vocale s. 61 , als germanische endung des acc. plur. fem. angesetzt worden ${ }^{1}$ ), nur lässt er das $\bar{u}$ in germ. $\bar{o}$ ubergegangen sein (die bezeichnung der germ. langen vocale und der nasalierung ist bei ihm eine andere, compliciertere), Mablow fuhrt aber nicht die länge des ahd. $-\hat{a}$ auf die nasalierung zuruck.

Dem wechsel von $\bar{a}$ und $\bar{o}$ im feminin gegenitber haben wir im masculin und neutrum einen ursprunglichen wechsel zwischen einem $\bar{e}$ (aus $-e-+$ vocal) der schwachen casus und

1) Mahlow wird auch recht darin baben, wenn er s. 128 das ahd. -a des nom. acc. pl. masc. taga, das nicht das urspr. -os germ. -vz des nominativs sein, auch nicht aus germ. -anz entstanden sein kann, auf germ. $-a^{n} z$ mit nasaliertem vocal zurïckfiihrt. L̇s künnte vielleicht die lautregel aufigestellt werden, dass urspriinglich auslautende gruppe vocal $+u s, m s$ in unbetonter silbe germ. nasalierter vocal $+z$ werde. Sonst hat sich zu einer zeit, wo noch kurzer nasalierter vocal iw germanischen auslaut bestand, acc. stainan (oder noch - (1)n) fur das spiitere staina, der acc. plur. $-a^{n} z\left(-o^{n} z\right),-i^{n} z,-u^{n} z,-\bar{a}^{n} z$ nach dem acc. sing. $-a^{n}\left(-o^{n}\right),-i^{n},-u^{n},-a^{n}$ gerichtet. - Das nordische $-a$ des acc. plur. úlfa kann aber nicht, wie Mahlow will, aus $-a^{n} z$ entstanden sein, da das $z$ nicht spurlos geschwunden wäre: dieses an. $-a$ ist germ. $-i^{n}=$ slav. $\dot{y}$, vlükȳ, sanskr. -än, vrkān, aus urspr. -ïn (unverkürzt an. páa acc. pl. = slav. $t \bar{y}$ skr. $t a \bar{n} n)$, ebenso ist das an. $-i,-u$ des acc. plur. masc. der $i$ und $u$-stämme germ. $-i^{n},-\bar{u}^{n}=$ slav. $-i,-\bar{y}$, skr. und urspr. $-i n,-u u$. Diese endungen des acc. plur. masc. werden die ältesten sein, und die eben angesetzten auf $-z$ werden sich zum acc. sing. gebildet haben nach der analogie der formen der consonantischen declination, germ. acc. sing. *-un, acc. pl. -unะ. 
einem " (aus -o- + vocal) der starken. Sahen wir aber beim fem. einen übergrift' des $\bar{a}$ der schwachen casus in das gebiet ler starken, so sehen wir hier umgekehit das $\bar{o}$ der starken casus auf kosten des $\bar{e}$ sich ausbreiten. Ueber das $e$ und $\bar{e}$ ler schwachen casus s. F. de Saussure, Syst. prim. 90 f., 118 anm., vf. Engl. stud. III, 152. Den grund des in den schwachen casus eintretenden $e$ s. u. im excurs.

Die urspringliche endung des instrumentals sing. ist $-\bar{e}^{-1}$ ), erhalten in got. $p \hat{e}, h v \hat{e}$, und im altengl. -e im subst. und adj., $d a z e$, $\bar{\delta} \hat{o} d e$. Im latein. sehen wir an die stelle dieses $-\bar{e}$ ein aus den starken casus eingedrungenes $-\bar{o}$ (lat. $-\check{o}$ ) getreten (yuando, ho-die): dieses selbe $-\bar{o}$ haben wir in dem deutschen $-u$, instr. as. dagu ahd. $\operatorname{lagu}{ }^{2}$ ) Sievers findet Beitr. V, 136 in dem as. ahd. $-u$ den ablativ und in dem ae. $-e$ einen mit dem $m$-suffix gebildeten instrumental. Für dieses sehe ich in der form keine möglichkeit, jenes anzunehmen (wie es auch Paul tut, Beitr. II. $339 \mathrm{ffi}$., IV, 454) ist der form wegen unnötig, der bedcutung wegen unmöglich, denn der casus auf $-u$ ist ein wirklicher instrumental, steht nicht wie ein ablativ bei präpositionen der entfernung (mit rehtu, aber fon thesemo dage). Die beilen formen des instrumentals verhalten sich $\mathrm{zu}$ einander, wie in lateinischen ablativ das ältere certe (facilume $\bar{e}$, osk. -id) und das jüngere certō. Ebenso haben wir im gen. plur. als schwachem casus ursprünglich die endung $-\bar{e} m$, germ. $-\bar{e} n$, got. lagê, aber wie im griech. $-\infty \nu$ der $o$-laut der starken casus eingedrungen ist, so haben wir auch im gen. plur. ahd. tago as. dago ae. $d a \not ̧ a$ die endung -̄̄n aus -ōm. Genau dasselbe verhältnis sehen wir im gen. sing. got. pis, dagis mit dem stammauslaut -e-, gegentiber dem ae. pass, drzes, as. dugas (neben dages) mit dem stammauslaut - $a$-, älter -o- vor dem $-s$, wie in griech. -oco aus -oøjo für esjo (liber das suffix s. u.). Im locativ sing. auf urspr. - ei (s. Saussure 91), welcher casus aber im gotischen fehlt, haben wir im ubrigen germanischen die endung -ai aus -oi, ahd. tage as. dage ae. daze an. uiln

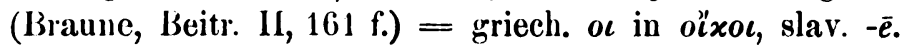

') Zeugen fïr -e aus dem indoiran. s. Joh. Schmidt, Kuhns zs. $\mathrm{XXV}, 9 \overline{\mathrm{i}}$.

2) Wegen solcher differenzen zwischen den germ. dialecten s. den schluss des allfsatzes.

Buitrüre aur geschichte der deutschen sprache. Vil. 
Neben diesem locativ und neben dem ablativ got. dug" aus urspr. -êd, pamma, blindamma aus urspr. -smēd kann der ahd. as. dativ der pronominalen declination auf $-m u$, $-m o$ kaum etwas anderes sein als der alte dativ auf $-s m \bar{o} i$, sankr. -smāi mit dem $-\bar{o} i$ in $\lambda \dot{v} x a ̣$. Es steht kein anderer uns bekannter casus mehr zur verfügung. Urspr. $-\bar{o}$ wird ahd. as. $-u$, urspr. $\bar{o}+$ consonant $m$ oder $s$ wird $-o$, was soll aus urspr. $-\bar{o}+$ consonant $i$ wol anderes werden als ein -o oder $-u$ ? Urspr. $-\bar{o} m$ oder $-\bar{o} s$ wird offenes $-o$, das nicht zu $-u$ wird, urspr. $-\bar{o} i$ vielleicht geschlossenes $-o$, das in der schreibung älter als $-u$, jünger als -o erscheint (genaueres s. Braune, Beitr. II, 158). Durch eine reihe von beispielen lässt sich diese entsprechung fụr altes $-\bar{o} i$ nicht erweisen, weil solche beispiele fehlen. Paul sieht in dem casus auf $-m u,-n_{i} o$ den ablativ, es wäre dies also ein ablativ auf $-s m \bar{o} d$ mit dem eingedrungenen $\bar{o}$ für $\bar{e}$. Im altsächsischen müste das $-u$ nach zwei vorausgehenden silben schwinden. Wir finden dasselbe nun in einigen fällen wirklich geschwunden, dat. sing. kristinum: dieser dativ auf $-m$ könnte ein ablativ auf $-s m \bar{o} d$ sein. Es wird aber wahrscheinlich ein ganz anderer casus sein, der instrumentalis II (sociativ) auf urspr. $-m i=$ slav. $-m \check{\imath}$, den wir im friesischen, engliscben und nordischen durchaus als vertreter des dat. sing. im masc. der pronominalen declination finden (altnord. peim ae. $\boldsymbol{b} \hat{\boldsymbol{e}} \boldsymbol{m}=$ slav. $t \bar{e} m \check{\imath}$, verschieden von altsächs. themu ahd. demo, altnord. øðrum ae. ôtrum = as. ăbrum aber verschieden vom dativ as. -emu, -umu). Dass dieser $m$-casus auch im deutschen bestanden hat lehrt und das einfache $m$ in ahd. demo as. themu: der dativ urspr. tesmōi und ebenso der abl. tesmōd hätte cine form mit $m m$ geben mussen, das einfache $m$ ist von diesen zweiten instrumental heribergenommen.

Ist der ahd. as. dativ der pronominalen decl. auf $-m u$, -mo ein ursprünglicher dativ, dann muss ebenso im feminin der dativ altnord. giofu, giof altsächs. getu, -o ahd. gebu, -o und in der pronominalen declination as. ahd. -ru, -ro ein dativ auf $-\bar{o} i,-s j \bar{o} i$ sein, neben dem locativ ae. zife und in der pronominalen decl. ae. blindre an. blindri aus urspr. -ai und danach gebildetem -sjai. Das - $\bar{o} i$ statt $-\bar{a} i$ (mit dem $-\bar{o}$ der starken casus) bat sich gebildet unter dem einflusse des $-\bar{o} i$ des dat. masc., genau ebeuso wie im gen. plur. fem. das ursprüng- 
liche - $\bar{a} m$ des schwachen casus nach dem urspr. $\bar{o}$ des nom. sing. und plur. zu $-\bar{o} m$ umgebildet ist, griech. $-\infty v$ germ. $-\bar{o} n$, unter mithulfe des $-\bar{o} m$ für $-\bar{e} m$ des gen. plur. masc. (so nicht notwendig im gotischen, dessen $-\hat{o}$ ein gen. plur. germ. $-\bar{a}^{n}$ aus $-\bar{a} m$ sein kann, wenn ein germ. $-\bar{a}^{n}$ wie wir es oben angesetzt laben got. - $\hat{o}$ und nicht got. - $\hat{a}$ wird). Das got. -ai des dat. sing. gibai, blindai, pizai ist das -ai des locativs. gibai könnte möglicherweise der dativ auf $-\bar{a} i$ sein, doch nur dann wenn die oben gegebene erklärung des ahd. as. $-u(-0)$ an. $-u$ aus $-\bar{o} i$ falsch ist, denn diese erklärung setzt voraus dass ursprunglich auslautendes $-\bar{a} i$ im gotischen $-\hat{o}$ oder vielleicht $-\hat{a}$ wird. Dass der gotische dat. fem. auf -ai ein locativ ist, dafür spricht vor allen dingen blindai, denn zum gen. blindaizôs muste der ursprlingliche dativ blindaizai lauten, wenn - $\bar{a} i$ gotisch -ai wird. Der locativ pizai ist entstanden indem nach dem verhältnis dativ -āi : loc. $-a i$ zu einem ursprünglichen dativ auf $-s j a \bar{a} i$ ein loc. auf -sjai geschaffen ward. Das -sjai des locativs finden wir im gotischen nur noch beim pronomen izai, pizai, noch nicht beim adjectiv, in den jungeren dialecten, im altnord., altengl, altfiries. auch beim adjectiv. Das $j$ des $-s j$ - ist in pizôs, pizai natiirlich durch ausgleichung mit dem $s$ des gen. plur. verloren gegangen, ebenso wie aus dem gen. plur. das ursprünglich nur dem gen. plur. masc. zukommende ai des gen. sing. fem. blindaizôs stammt. 1)

Die westgermanische endung des nom. sing. fem. der $n$-declination ahd. as. $-a$, afr. ae. $-e$, as. tunga, ae. tunge, gegeniber dem got. $-\hat{o}=$ griech. $-\infty \nu$ lat. $-o$, erklärt Osthoff, Morph. unters. I, $255 \mathrm{ff}$. aus einem $-\vec{e}^{n}$, das ursprunglich nur bei vorhergehendem $j$ berechtigt gewesen wïre: von den doppelformen ohne und mit $j$, die ahd. zu heissen hätten masc. hano, ${ }^{*}$ erbia, fem. ${ }^{*}$ zungo, redia, hätte beim fem. die $j$-form, beim masc. die $j$-lose form den sieg davon getragen. Den ostgermanischen ausgang des nom. sing. masc. erklärt Osthoff s. $260 \mathrm{f}$. durch die einwirkung des artikels. sa *blindô neben

1) Als dative erklären die formen anf $-m o$, -ro auch Leskien, Decl. 127 f. und Mahlow, s. 51, 93, die aber beide auch got. pamma als dativ fassen. Gegen die herleitung des $-u,-o$ von $-\bar{o} i$ streitet Paul, Beiträge IV, $461 \mathrm{f}$. 
sô hlindô habe sich zur herstellunğ der harmonie zu sa blinda umgestaltet. Dies letzte ist des nordischen wegen nicht möglich: das altnordische $-i$ kann nicht, wie Osthoff will, aus (oder älterem -o) in unbetonter silbe entstanden sein, sondern ein nordisches $-i(-e)$ ohne vorhergehenden umlaut ist aus germ. $-\bar{e}$ oder $-a i$ hervorgegangen.

Meine ansicht über den ursprung der formen des nom. sing. der $n$-declination könnte ich zwar in kürzerer weise vorbringen, aber es scheint mir dem gegenwärtigen und noch darüber hinausgehenden zwecken dienlich zu sein, wemn ich zuvor meine ansicht über die entstehung des $o\left(=a_{2}\right)$ in den indogermanischen dialecten darlegre, obwohl ich dazu auf den ältesten dem indogermanischen sprachforscher überhaupt erreichbaren sprachlichen zustand zurückgehn muss.

\section{Excurs: Die entstehung des o.}

Die ursprüngliche gestalt der indogermanischen wurzcl, d. h. natürlich des indogermanischen wortes, genauer nomens, war die: die wurzel war zweisilbig mit inuerem vocal $a$ und auslautenden vocal $a$, nach den consonanten bilitteral wie $B u ́ R a$ (träger) $^{1}$ ) oder trilitteral (mit innerem $i, u, r$ oder nasal + cons., oder $\left.A, E^{2}\right)$ vor oder nach cons.) wie DaRla (blickend), VaIDa (sehend), DaIVa und I IaVa (glänzend, hinmel), DallAa (iöndigend) (diese trilitteralen waren noch früher dreisilbig, drei a enthaltend, daraca, vajada, dajava, denn sie sind componiert, z. b. damaAa aus dama $+A a$, und es findet ein wechsel statt zwischen der zweisilbigen wurzel mit dem ersten und mit dem zweiten $a$, wie daiva und djava).

1) Die aspiraten gelten als einfache cousonanten, ebenso im anlaut $s+$ cons. (Im semitischen kann möglicherweise ein in historischer zeit unerhörtes älteres anlautendes $s k s t s p$ zu $k t p$ geworden sein.)

2) Ueber die consonanten $A, E^{\prime}$ vgl. Engl. stud. III, 150 f. Es waren consonanten von der art, wie wir sie in historischer zeit ganz gewöhnlich mit dem vorhergehenden vocal verbunden in einem langen vocal sich verlieren sehen (z. b. $h$ oder gutturales $r$ ), wahrscheinlich gutturale von der art der semitischen, $A=$ alef, der tonlose guttural. verschlnsslaut, und $E^{\prime}$ wahrscheinlich der entsprechende tönende verschlusslaut. $A$ künnte in den meisten fïllen auch ein gutturales $r$ gewesen sein, natiirlich künnen in diesen elementen verschiedene lante 
Diese wurzelwörter liegen historisch in vier gestalten vor, dic, wie sich uns ergeben wird, aus vier nur durch die betonung unterschiedenen urgestalten desselben wortes hervorgegangen sind. Die betonung der grundsprache war die musi-

stecken, die fïr unsern blick zusammengefallen sind. Der consonant $A$ hatte die eigenschaft, ein vorhergehendes $e$ in $a$ und ebenso den schwarocal $e$ in den schwavocal $a$ zu wandeln (oder auch einem hochtonigen $a$ der grundsprache, das sonst $e$ geworden wäre, und einem tonlosen $a$, das sonst $e$ geworden wäre, den $a$-lant zu wahren): ${ }^{*} e A$ wird ${ }^{*} a A$, worius $\bar{a}, * \| d$ wird $\bar{o}$. Der consonant $E$ fliesst einfach mit dem vorhergehenden vocal $e$, $\sigma$ in dessen länge zusammen. Es verhalten sich also wie ei $: \omega i: i$, er $: o r: r$ so $\bar{a}: \bar{o}: A$. $i: \bar{o}: E$ (s. F. de Saussure, Syst. prim. $136 \mathrm{ff}$.). Die zeichen $A, E$ in tonloser stufe bezeichnen selbstlautendes $A$ oder $E$ wenn die consonanten $A$ oder $E$ stimmtonlaute (wie b. b. gutturales $r$ ) waren, dagegen einen aus schwa $+A$ oder $E$ ' hervorgegangenen schwavocal wenn $A$ oder $E$ gerïuschlaute waren. $A$ in tonloser stufe wird in sanskrit ohne erkennbare regel entweder $i$ oder $1: i$ entsteht vielleicht ans $\mathcal{A}$, schwa mit folgendem $A$, wenn das mit $A$ bezeichnete element ein geräuschlaut, $i$ dagegen aus selbstlautendem $\boldsymbol{A}$, wenn das mit $A$ bezeichnete element ein sonorlaut war.

F. de Saussure trennt $A$ und $E$ nicht, stellt aber dem $A$ ein anderes element \%ur seite, das er o schreibt, dessen ablautstufen $\bar{o}: \bar{o}: \emptyset$ sein sollen. Saussure sieht aber in diesen elementen vocale, nicht wie ich gutturale geräuschlaute oder event. ein gutturales $r$.

Saussures element ? hat in den meisten der wïrter, denen er das 0 beilegt, sicher nicht bestanden, und vielleicht hat das element $\varrho$ und also eine ablantreihe $\bar{o}: \bar{o}: 0$ iiberhaupt nicht existiert. In den weitaus meisten fällen gehört nämlich dieses " in die $A$-reihe und ist nichts anderes als das von Saussure selbst s. $11: 3 \mathrm{f}$. in erwägung ge\%ogene, aber schliesslich abgewiesene, 'une simple altération gréco-italique de $A$ '. Es fallen hierher namentlich zahlreiche griechische " fiir erwartetes a, die ax- von der wurzel ac ("̈xps, ong ós neben orespis. Diese o fiir a sind enstanden durch inderung des

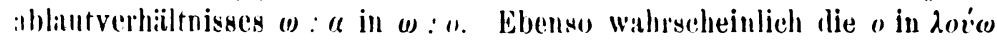

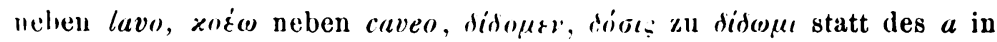
lat. damus, dator. In griech. d(1)-:do- aus dii : do: dA hätte also das griechische die stufe $d a$ aufgegeben und dann den ablaut $\delta \omega: \delta \alpha$ in do : do geändert. Ebenso sind andere und vielleicht alle $i: 0$ : $o$ ent-

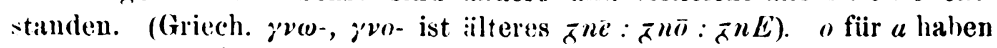

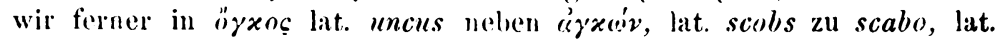
"plus wenn äqvos verwant ist, griech. "̈бor lat. oculus. Dass diesem o im sanskrit a entspricht, der regelrechte vertreter von $A$, ist völlig in der ordnung, ebenso dass diese $o$ in armenischen durch $a$ gegeben werden, lal geben, akn auge ( $A$ ist armen. $a$, griech.-lat. $o$ armen. $o$ ). Andere dieser $o$ sind entstanden durch umgestaltung des ablauts $\bar{o}:-$ in $\delta: o$. 
kalische (nach Verners bezeichnung 'chromatische'), denn alle älteren dialecte, von denen wir bestimmte nachrichten ther die betonung haben, das sanskrit, griechische, lateinische ${ }^{1}$ ), hatten die musikalische betonung. Die verschiedenen stufen der betonung benennen wir fur die grundsprache am besten mit den namen des ältesten indogermanischen dialects, der auch die ursprtingliche betonung im ganzen am treuesten festgehalten hat: udātta (hochton), anudātta ('unton') und svarita. Der svarita ist ein doppelter, der abhängige (tiefton) und der selbständige (hochtiefton). Die lateinischen namen sind fur uns nicht geeignet, weil wir mit ihnen den gedanken an exspi-

S. u. im einzelnen die besprechung der meisten der genannten und anderer wörter. Immerhin bleibt es möglich, dass in einzelnen fällen ein element wie Saussures $o$ bestanden hat, das mit vorhergehendem $c$ und $o$ zu langem $\bar{o}$ verschmolz: ich wïrde dann fïr dieses element den wert eines gutturalen $r$ vermuten. Ich sehe indessen kein wort, fiir welches die annahme dieses dritten elementes notwendig und die erklïrung des $o$ durch geänderten ablaut unmöglich wäre. Ich werde daher im folgenden Saussures $g$ genau so behandeln, wie Sanssure selbst das $E$ behandelt.

Sollte in einzelnen fällen ein $\bar{a}$ einem $\bar{e}: \bar{o}$ gegentiberstehen, also

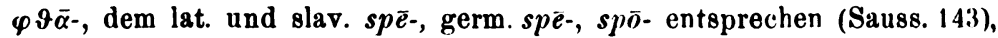
dann wïde das nebeneinanderstehen der consonanten $A$ und $E$ in speAneben spe $E$ - absolut nichts anderes sein, als das nebeneinanderbestehen anderer consonanten, wie in den von Saussure 142 und 152 bemerkten fällen meE- neben med- messen, geA-neben gem- gehen, steA-neben stel- stehlen (composition einsilbiger urwurzeln mit verschiedenen elementen, $M a-E a$ und $M a-D a, G a-A a$ und $G a-M a)$.

1) S. die stellen bei F. Schöll, de accentu linguae latinae veterum grammaticorum testimonia (Acta soc. phil. Lips. VI) 8. $i 1-215$, besonders nr. 2a, 2 c, 3, 18, 21, Mart. ('apella 3, 6s, Ilomed, Keil 2, 430, die flir jeden, der nicht, wie Sch8ll selbst, sich Uberhaupt nichts anderes denken kann als die monotonie and den exspirationsictus des gegenwärtigen nhd., die musikalische natur des lateinischen accents beweisen.

Dass der sprachlaut drei eigenschaften habe, eine dauer, höhe und stärke, sagte lange vor Kräuter M. Terentius Varro ( 2 a bei Schöll : 'Scire autem oportet vocem sicut omne corpus tres habere distantias, longitudinem, altitudinem, crassitudinem .... altitudinem discernit accentus, cum pars verbi aut in grave deprimitur aut sublimatur in acutum. crassistudo in spiritu est: omnes voces aut aspirando facimus pinguiores aut, sine aspiratu pronuntiando, tenuiores'), der aber nicht als vierte dimension, wie Kräuter (nach den physiologen) als vierte 'eigenschaft' den 'klang' hinzufügte, also das wesen des sprachlautes selbst. 
ratorische betonung verbinden, und weil, wenn wir den namen 'gravis' für den abhängigen svarita brauchen würden, uns ein ausdruck für den anudātta fehlt. Dass ein ursprüngliches $a$ durch den udātta $e$ wird und dass reducierîe silbe von ursprünglichem anudātta stammt, ist schon bekannt (wenn man sich auch wol die betonung, die diesen unterschied schuf, meistens als exspiratorische denkt), s. Verner, Kuhns zs. XXIII, 133: nicht allgemein bekannt ist, dass die $o$-färbung von ursprünglichem svarita herrührt. Den udātta oder hochton (= acut, griech. ó $\xi \varepsilon i \alpha$, scil. $\pi \rho o s \varphi \delta i ́ \alpha)$ bezeichne ich durch ', den anudaitta oder unton durch sein gegenstück, den svarita durch ', und zwar den abhängigen svarita (= gravis, griech. $\beta \alpha \rho \varepsilon i \alpha$ ) durch ein ' auf dem vocalzeichen $(\grave{a})$, den selbständigen svarita, d. i. svarita mit vorhergehenden udātta in éiner silbe (= circumflex $\left.{ }^{1}\right)$, griech. ó $\left.\xi v \beta \alpha \rho \varepsilon \tilde{\alpha} \alpha\right)$ durch ein ' dem vocal-

1) Sievers ist im irrtum, wenn er den geschliffenen accent, der die diphthongierung des $\dot{e} \bar{o} z u$ ia, $u a$, die westgerm. consonantendehnung, das aufkommen des svarabhaktivocals bewirkte, mit dem circumflex identificiert. Der lat. circumflex (Varro, no. 36 'apud nos flexa, quoniam primo erecta rursus in gravem flectitur') gleich der griech. ${ }^{\prime} \xi v \beta \alpha \rho \varepsilon i \alpha$ $\pi \varepsilon q \sigma \pi \omega \mu \varepsilon \dot{\varepsilon} \eta$ oder $x \xi x \lambda \alpha \sigma \mu \dot{\varepsilon} \nu \eta$, dem indischen selbständigen svarita, ist vielmehr' gleich dem 'gestossenen accent' des litauischen, wie ihn Kurschat beschreibt, dagegen der 'geschliffne accent' ist gleich der griechischen iv' $\tau \alpha \nu \alpha x \lambda \alpha \zeta \sigma_{0} \dot{\varepsilon} \cdot \eta \eta$ des Glaukos v. Samos (s. Keil gr. lat. 4, 530), dem vom tieferen zum höheren tone aufsteigenden accent aller griechischen betonten langen vocale und diphthonge, die nicht den "tragen, $\varrho \omega^{\prime} u \eta$, diphth. $\alpha \dot{i}, \alpha \dot{v}$ (Kurschats lit. $a \tilde{\imath}, a \tilde{u}$, hier $a i^{\prime}, a u^{\prime}$ gegeben, ist $=$ griech. $\alpha i, \alpha \dot{i}$, nicht $=$ gr. $\alpha i, \alpha \tilde{v})$, der dem lateinfschen unbekannt war. Der geschliffene accent bewirkt diphthongierung (wie die des durch die sog. ersatzdehnung entstandenen langen geschlossenen $\&, O$ zu $\&$, or), der gestossene accent oder circumflex dagegen umgekehrt die verengung von diphthongen. Der lat. diphthong in aidem war, worauf schon die schreibung mit ae hinweist, nicht griech. ai $(\rho \mid \bar{F})$ mit geschliffenem accent, sondern ein $a i$ $(\rho \rho)$ mit gestossenem oder circumflex (= gr. $u i)$. Schïll, der jeden unterschied zwischen dem antiken und modernen accent läugnet, geht gegen sämmtliche zeugnisse der grammatiker an, indem er dem griechischen und lateinischen den eirenmflex abspricht (was P. Langen, Fleckeisens jahrb. $1876,619 \mathrm{ff}$, nur gegeniiber dem lateinischen tut), bloss weil er sich einen unterschied zwischen $\dot{\omega} \tilde{\omega} \mu \alpha$ und $\dot{\omega} \omega \mu \eta$ nicht denken kann und dergleichen unterschiede für 'argutiae' hält [jenes ist $\widetilde{\rho} \rho d$, dieses $\hat{\rho} \bar{F}$ ]. 
zeichen folgend ( $u$, bei folgendem $i u r n m$ rult der svarita auf diesem, ai, aù, ar', an', am', der udātta selbst bleibt vor diesem svarita unbezeichnet). Ein hochtoniges $a$ der grundsprache wird in den indogerm. dialecten $e$, ein untoniges $a$ schwindet oder wird schwa $(e)$, ein svaritiertes is wird $\left.o^{\prime}\right)$, ai aic ar ai ' wird oi ou or on, $a^{\prime}$ wird $\bar{o}$.

Wir könnten, wenn wir diese accente hinzudenken, die formen der grundsprache, wie sie Schleicher schrieb, in den meisten fällen bestehen lassen, denn die wirkliche grundsprache wird allerdings dieser friiher geschriebenen immer noch ähnlicher gewesen sein, als derjenigen, die wir seit Brugman und Collitz schreiben. Doch ist die jetzt iibliche methode unverwerflich, wenn wir es nur wissen, dass wir nicht die grundsprache reconstruieren, sondern dasselbe tun, wie wenn wir aus den romanischen dialecten eine grundsprache reconstruieren würden, die in jeder wortform die gremeinromanischen lautgesetze durchgefuhrt zeigte, z. b. clam $\bar{a}^{\prime} t e,-$ las statt $c l \bar{a} m \bar{a}$ tae, $-t \bar{a} s$.

Die vierfach verschiedene musikalische betonung des zweisilbigen wortes der grundsprache war die folgende:

I. Die erste silbe die zweite

trägt den den

udātta, svarita : dárcia, histor. dérco-

b) anudātta, udatta : d,arcá, , drcé-

2. a) svarita, anudätta : diar ca, " iorc-

b) anudātta, anudātta : darcia, " drc-,

die letzte form vor udätta des suffixes. Eine fünfte form, die des vocativs (und, was ursprlunglich dasselbe, der 2. sing. impl).), hist. derce, bleibt als singulärer fall hier unheriicksichtigt.

Dieselbe vierheit haben wir beim antritt (historisch) unsilbiger suffixe. Dic formen sind, nit setzung der historischen $e,-, o$ fur $\dot{a}, a, i \iota$ :

Wurzel- Stammauslaut nach antritt von
$\begin{aligned} \text { 1. a) } & i \\ \text { b) } & - \\ \text { 2. a) } & \dot{0}\end{aligned}$
$\begin{array}{llll}\operatorname{suffix}-A & -i, & -u, & -n, \\ (* o A)-\overline{0} & -o i & -o u & -o n\end{array}$
$-t, \quad-s:$
b) -
$\left({ }^{*} i A\right)-\bar{a}^{\prime}-\quad-\ddot{i}-$
$\begin{array}{ll}-o u & -o n \\ -e \dot{e} u- & -i n-\end{array}$
$-o t \quad-o s$
$-i t-i s-$

$\begin{array}{ll}-A & -i \\ -A-^{\prime} & -i-^{\prime}\end{array}$
$-u$
$\begin{array}{lll}-n & -t & -s \\ -n-{ }^{\prime} & -t-^{\prime} & -s-^{\prime} .\end{array}$

1) Vgl. Gust. Meyer, Kuhns zs. XXIV, 22; f. Mehr abweichend Paul, Beitr. VI, 112 f. Paul nimmt a. a. o. an, dass dic vocalstufen auf 
Diese suffixe waren in der grundsprache einsilbig, $-d$ war urspr. $-A a$ (spir. lenis $+a$ ), ebenso $-i-u$ urspr. -ja $-v a,-r-n-m$ $-\ell$-s urspr. -ra -na -ma -ta -sa etc. Nehmen wir diesen IIrsprünglich folgenden vocal hiuzu, dann gewinnen wir für die dreisilbigen wörter der grundsprache sechs verschiedene formen, es bestand nämlich eine form (3. a), in welcher das suffix als ursprünglich selbständiges wort seinen selbständigen accent als svarita wahrte, den vorhergehenden svarita in den anudätta wandelnd (dárc a-tì aus dírcì tá).

$$
\begin{aligned}
& \text { II. (Grund- Wurzel- Wurzel- } \\
& \text { form) vocal auslaut } \\
& \text { 1 a) }\left(\begin{array}{lllllll}
\operatorname{darc} \dot{a}-t a & i & -0- & -n & -m & -t & -s
\end{array}\right. \\
& 1 b)=4 a) \quad d \text { arcí-ta } \quad-\quad-i-\quad-n o-\quad m o-\quad-t o-\quad-s o- \\
& \text { 2a) dar } c, a-t, a \quad b \quad-\quad \begin{array}{lllll}
-n & -m & -t & -s
\end{array} \\
& \text { b) darca-ta - - }-\dot{n}-^{\prime} \quad-\dot{m}-^{\prime} \quad-t^{\prime} \quad-s^{\prime} \\
& \text { 3a) diarc a-ta } i \quad-\quad-n o-\text {-mo- }-t o-\text {-so- }
\end{aligned}
$$

\begin{tabular}{|c|c|c|c|c|c|}
\hline & & Wur & Wurzel- & Suftix, & \\
\hline a) & (urru & vocal & atuslaut & $-a-r a$ & $\begin{array}{c}-m a-n a \\
-m n\end{array}$ \\
\hline b) & darca-tara & _- & - & $-\dot{t} r-$ & $-m \dot{n}-$ \\
\hline l) & $\operatorname{darc} a-t a r a$ & $e^{\prime}$ & - & -tor & $-m o n$ \\
\hline a) & d, arc a-tara & - & - & -tiro- & neine \\
\hline & d arc $a-t a r a)$ & - & -. & $-\operatorname{tre} e^{-}$ & $m n$ \\
\hline
\end{tabular}

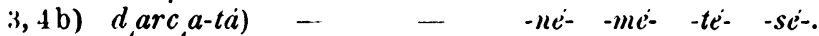

In den beiden ersten formen bleibt der auslautende vocal der ursprünglichen wurzel gewahrt, in den vier tulorigen schwindet er.

Beim antritt zweisilbiger (componierter) suffixe, also in ursprünglich viersilbigen wörtern waren noch mehr formen der betonung möglich. Die tiblichsten und für uns wichtigsten sind:

Die regeln aber dic aufeinanderfolge von $e$, o und sehwund des vocals sind:

1) Das o hat scine stelle in urspringlich letyter silbe eines mehrsilbigen wortes oder wo in der folgenden silbe ein $a$ seeschwunden ist (Engl. stud. III, 154 unten), d. h. fiur die grundsprache: auf svarita folgt stets anudatta (bhérō qéoo aus blírì- $A, a)$.

2) Einem $o$ in der eniung oder dem suffixalen teile eines wortes geht stets ein $e$ vorauf, d. h. vor dem abbängigen

einer abstufung des exspiratorischen accentes, nicht eines wusicalischen beruhen, ferner lässt er, :ihnlich wie friiher Iloltzmann, das 0 unter dem hauptton, das $e$ unter dew nebenton entstehen. 
svarita steht stets udatta, entweder unmittelbar oder durch einen auf dem ursprỉnglichen wurzelauslaut ruhenden anudätta von ihm getrennt.

3) Dem $e$ geht stets schwa oder schwund des vocals in allen vorhergehenden silben vorauf (F. de Saussure, Syst. primitif s. 236 unten), d. h.: alle silben vor der mit dem udātta versehenen tragen den anudātta. Da der selbständige svarita die vereinigung des udātta mit dem svarita in einer silbe ist, so steht auch vor diesem anudātta (dont-s aus adarita-s $\left.a^{1}\right)$.

Fur die behandlung des svaritierten $a$ ist noch eine regel zu geben:

$a$ in ursprünglich offener silbe wird durch den svarita gedehnt, wird also $\bar{o}$, wenn dem svarita ursprünglich doppelter anudātta folgte. Daher dorc-s (anblick) aus därc,a-s a, aber $p \bar{o} d-s$ (fuss) aus pàda-sa. -mon, -tor aus -màn $a$, tìr $a$ wird $-m \bar{o} n-$, tōr - sobald noch ein anudātta folgt, also acc. masc. $-m \bar{o} n-m,-t \bar{o} r-m$ aus -màn $a-$, tàr $a-+m a$.

Alle anstatt dieses $\bar{o}$ sich findenden kurzen $o$ wie in griech. $\pi \circ \delta$-, acc. $-\mu o v \alpha,-\tau o \rho \alpha$ stammen (wie dies schon von Joh. Schmidt, Kuhns zs. XXV, $13 \mathrm{ff}$. für die wichtigsten fälle gezeigt ist) aus der schwachen stufe, in welche sie geraten sind dadurch dass das verhältnis $\bar{o}: e$ (-mōn-:-mén-) oder $\bar{o}:-$ ( $p \bar{o} d-: p e d$-) durch das verhältnis $\bar{o}: o$ ersetzt ward. Wir werden dies im folgenden im einzelnen sehen. ${ }^{2}$ )

Recht hatten also diejenigen, welche dem indoiran. $\bar{a}$ in offner silbe den wert des ' $a_{2}$ ' beilegten, Brugman, Osthoff, de Saussure, recht ebenfalls diejenigen, welche dasselbe $\bar{a}=$ europ. $\bar{o}$ setzten, Joh. Schmidt, Collitz und ich, unrecht aher Latten diese indem sic jonon wort des indoiran, $\bar{a}$ lengneten, jene weil sie behaupteten, das $\bar{a}$ sei $=$ europ. $o$.

In der grundsprache fand in der flexion eines jeden wortes eín accentwechsel und also in der jungeren sprache ein ablaut

1) Im suffix -nla, ebenso in -nka, ist die aufeinanderfolge der consonanten so alt wie die des $n, r, i, u+$ cons. in wurzel bhandha, darca, vaida etc.

2) Die anwendung des gesetzes auf das verbum s. in der anmerkung am schlusse dieses abschnittes. 
statt. Der accent wechselt \%wischen den je zwei oben durch a) und b) bezeichneten accentlagen: die accentlage a), die als die normale anzusehen ist, gilt fur die starken, b) für die schwachen casus. ${ }^{1}$ )

Alle wörter mit dem vocal $o$ in erster silbe in den starken casus hatten ursprünglich den accentwechsel 2. So 1) alle historisch einsilbigen wurzelwörter (vgl. Kuhns zs. XXIV, 519 f.), die in zwei gruppen zerfallen a) solche von wurzeln auf $n, r$, $i, u+$ cons. wie dor'c-s anblick, voic-s haus, roick-s licht (urspr. raùk $a-s, a$, acc. $-m a$ ), gen. drcòs, vicòs, rukòs (aus $r$ aukì-s,a? $\left.{ }^{2}\right)$ ), b) solche von wurzeln auf einfachen cons. wie $p \bar{o} d-s$ fuss, $v \bar{o}^{\prime} k-s$ stimme, $g^{u} \bar{o}^{\prime} v-s \mathrm{kuh}, d j \bar{o}^{\prime} v-s$ (skr. $d j \bar{a} u s$ ) himmel, bhōrs dieb (gr. $\varphi \omega ́ \varrho)$ ), *krō'p-s dieb (gr. $x \lambda \omega \dot{\psi} \psi$ ) (aus urspr. pa'd $a-s, a, d j a^{\prime} v a-s, a$ oder dajà $a-s, a$, bhàr $a-s, a$ etc.), gen. pedòs, *ukòs, guvòs, divòs, *bhròs (aus p adà-s,a, vakìt-s,a etc.?). Die ursprïngliche schwache stufe dieser letzten formen ist erhalten in lat. pedis (griech. $\pi \varepsilon \delta-$ in $\pi \varepsilon \zeta o ́ s$ ), skr. divás gr.

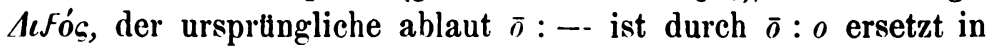

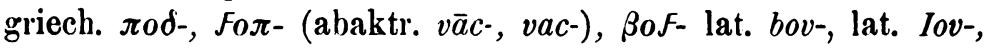
ebenso in griech. $\varphi \lambda \circ \gamma$ - und andern. Neben erhaltenem $\omega$ sehen wir dieses $o$ als schwache stufe in $x \lambda \omega \psi$, gen. plur. (bei Xenophon) $\left.x \lambda o \pi \tilde{a} v^{3}\right)$ - Weiter stellen sich hierher 2) alle historisch cinsilbigen abgeleiteten wörter, und zwar zur gruppe a) solche wie don't-s (,adarit $a-s, a)$, gen. dṇiòs (ad,anti $1-s, a$ ?), zur gruppe b) durch urspr. $t, a, s, a$ etc. von wurzeln auf ein-

1) Das 'stark' und 'schwach' der hier beibehaltenen iiblichen und hequemen ausdrilcke 'starke' und 'schwache casus' bitte ich nicht in dem sinne exspiratorischer betonung. sondern als 'rectus' und 'obliquus', 'uniblï̈ngig' und 'abhiingig' in syntactischem sinne auf\%ufassen.

$\left.{ }^{2}\right)$ Das suffix des genitivs war vielleicht urspr. -sa mit vorhergehendem svarita auf dem auslautenden a des stammes, so dass der genitiv ursprünglich dem nominativ mit $-s, a$ identisch, nur durch die betonung von ihm geschieden gewesen wïre.

3) In dem zahlwort 'vier' k"ctvi'r $E s$ geht dem selbstïndigen svarita entgegen dem oben gegebenen accentgesetz (3) der udättavocal $e$ vorauf. Das dem $t v v^{\prime} r$-, $t v r$ - voranfgehende $k$ " $e$ ist daher als selbständiges wort zu fassen $\left(k u_{i} i \vec{v} \vec{o} r_{E s}\right)$, die conjunction $k^{u} e^{i}$. In griechischen hat das $\tau \dot{\varepsilon}$ - den accent gewahrt. Neben dem regelrecht aus der schwachen stufe $k u \dot{t} t v r$ - stammenden $\tau \dot{\varepsilon} \sigma \sigma \alpha \rho \varepsilon c$ zeigt dor. $\tau \dot{\varepsilon} \tau o \rho \varepsilon \varsigma$ die schwache stufe tvor- init o (:o) fitir - (:o). 
fache consonanten ahgeleitete wörter wie das durch 1,a ahgeleitete wort 'nacht'. Dessen ursprtingliche flexion muss gewesen sein $n \hat{o}^{\prime} k t-s$ (aus $n a^{\prime} k a t a-s, a$ ), gen. ṇktòs. Die form nolit- ist genau ebenso enstanden wie die oben gesehenen Iov-, bov-, $\pi \circ \delta-$, foл-, indem zum $\bar{o}$ der starken casus in den schwachen ein $\cap$ sich einstellte, worauf die starke form von der schwachen verdrängt ward. $\left.{ }^{1}\right)$ Die starke form nōkt- ist noch sichtbar in ahd. muohturn mhd. nüehtern (s. u.).

Alle wörter auf -o hatten ursprïnglich den accentwechsel 1., dérco- drcé-. Die formen der starken casus waren ursprünglich paroxytona mit dem vocal $i$ in der stammsilbe. Wir haben 1) die ursprünglich zweisilbigen wurzelwörter. Die urspr. Hlexion war *virkò-s wolf, réukò-s weiss (grundspr. riukiı-s,a), gen. (nach der pron. decl.) vrkisjò, * rukésjò. ${ }^{2}$ ) In allen uns vorliegenden dialecten hat cine ausgleichung stattgefunden, $\lambda \varepsilon v x^{\prime}-\varsigma$ hat die wurzelsilbe der starken, den accent der schwachen casus verallgemeinert, $v r^{\prime} k o-s$ umgekebrt. Ebenso

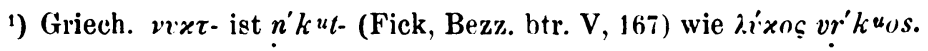

2) Oben ist als ending des gen. - $c^{i}-$ sjo angesetzt, obwohl es nicht die urspriingliche endung, sondern erst innerhalb der einzelnen dialecte, des indoiranischen und griechischen, aus der pronominalen declination heribergenommen ist. Die älteste genitivendung der wörter auf -o ist $-i-s \dot{o}$, d. i. der stamm mit dem pronomen urspr. $-s a$, nur durch die betonung vom nominativ unterschieden. Wir haben das -so des gen. in slav. $\dot{c} c-s i$, in griechischen in der endung $-o v$, denn IIomers neben einander herlaufende endungen -ov und -oso können unmïglich gleichwertig sein, sondern jenes ist $-\sigma-\sigma o$, dieses das $-o-\sigma j o$ der pronominalen declination. Endlich ist das gotische -is ahd. -es nicht - $e^{-s j o}$ sondern -eso, und die endung der librigen germ. dialecte (as. -as ae. $-\epsilon s$ an. $-s$ ) - - -so. In slav. de-so, im griech. und gerwan. (as. hoves, the's etc.) ist las - so fiir sju auch in die pronominale declination eingedrungen. - Das -sjo der prono. minalen declination ist dasselbe $s u+j a: t \dot{a}-s j i$ aus $t a \dot{a}-s, a-j a ̈$ ans $t \dot{a}-s \dot{a} j \dot{a}$.

Wie sich beim gen. die endung - $i s$ in der consonantischen, - $i s i$ in der o-declination, oder genauer dort $-s$, hier $-s i$ mit vorhergehendem stammauslaut, beides aus urspr. $-s a$, zu einander verhalten, so miissen die endungen aller obliquen casus in der consonantischen und in der odeclination eine verschiedene gestalt gehabt haben. Angenommen eine casusendung hatte in der cons. decl. die gestalt -ii (suffix $-j a$ ), so muste sie in der $o$-decl. als $-\dot{e}-j \dot{0}$ erscheinen. Die endung des instr. könnte in der cons. decl. $-\vec{\sigma}$ aus $-\dot{o} E$, in der $o$-decl. $-\hat{e}$ aus $-e^{E} \dot{o}$ sein. Dem -om des gen. plur. in der cons. decl. (suffix $-m a$ ) hätte in der 
sehr viele andere wörter (germ. z. b. deuza tier wie $\lambda \varepsilon v x o ́ s$ ). Dass der ausgleich oder die accentverschiebung, wenn sie auch gemeinindogermanisch ist, doch nicht in der grundsprache eingetreten sein kann ist klar, sie kann erst eingetreten sein nachdem vocalschwund und vocalfärbung, die wirkungen des alten accentes, längst sich festgesetzt hatten: wäre in der grundsprache auf eine bis dahin mit dem anudātta versehene silbe der udātta getreten, dann würde die sache ganz anders aussehen. Die formen der indogerm. dialecte weisen auf die flexion tévòs tuus, sévò-s suus (griech. $\tau \varepsilon \dot{c} \dot{c}_{\varsigma}$, ¿̇ós), gen. tvé-sjò,

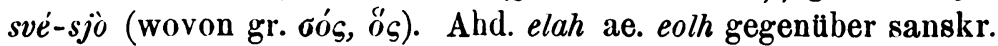
rça- weisen auf írcò-s, gen..rcé-sjò. Lit. bérža-s birke und sanskr. bhürza- sind ebenso bhérAzì-s, gen. bhrAzé-sjò. Got. gôd-s war ein *ghä'dhò-s *ghadhé-(AghAdhe-?, vgl. griech. áy $\alpha \vartheta o_{\varsigma}$ ?), dag-s war dhäghò-s dhaghé-sjò (vgl. fidur-dôg-s). Das friesische therp neben thorp $\mathrm{n}$. dorf weist auf térgu-m trgué- (s. Fick, Bezz. btr. V, 170).1) Manche vom nomen gelöste adverbien stehen noch da als zeugen der ursprlinglichen betonung, so sankr. sanăt zu sánas, aus abl. *snêd zu sénò-s. 2) Die mit einsilbigem suffix gebildeten wörter auf -no, -ro, -jo, -vo, -to, -nko etc. (II, 3 oder 4) und die mit zweisilbigem gebildeten, wie die auf -méno, -téro (III, 4), hatten denselben accentwechsel. Diese hatten daher -mné, - tré- in den obliquen casus, woher zahlreiche doppelformen, wie $\pi$ ó $\varepsilon$ cos und lit. katrìs. Die formen der indogerm. dialecte zusammengehalten zeigen uns ein part. auf -no svépnò-s schlaf, gen. supné-sjò (der accent in $\ddot{v} \pi \nu o s$ aus den starken casus). ${ }^{2}$ ) Griech. $\pi \varepsilon \varrho x v o ́ s$ und daneben $\pi$ axvós (Hesych.) ist spércnò-s slircné-sjo. Das pferd hiess écvò-s, gen. $c v \dot{e}-s j o$ : aus den obliquen casus stamnt,

$u$-decl. - $\dot{e}$-mi gegeniiberstehen miissen, das - $\dot{e}$-om des gen. plur. der $o$ decl. ist also formübertragung aus der consonantischen. Dem ablativ $-e^{-}-l o s$, lat. -itus, in der o-decl. (sutfixe -ta $+-s a$ ) muste in der cons. decl. $-\dot{j}-\iota s$, oder auch ohne vorhergehenden auslautenden stammvocal -téso entsprechen. Ich will indessen die urspriingliche gestalt der verschiedenen casussuftixe jetzt uicht weiter untersuchen.

1) [deino-dine- tag, in got. sin-tein-s, skr. -dina-, zu Kluge AFDA. V1 200.]

2) Vgl. Osthoff, Morph. unters. II, $11 \mathrm{ff}$. Lit. sa'pnas ist, glaube ich, nicht ein urspr. svopno-s, sondern hit vo ans $v e$ wie va'karas vesper, vasarà ver (s. Saussure (s)), var'das verbum. 
ausser dem accent, das griech. '¿xxos, $\left.\imath^{\prime} \pi \pi 0 \varsigma^{1}{ }^{1}\right)$ 'gelb' hiess (mit $l$ angesetzt) zhélAvò-s zhlAvé-sjò, von jenem lat. helvus ahd. gelo ae. zelo, von diesen an. gol-r. 'voll' (wie eben) pélAnò-s (urspr. pár $A a-n \grave{a})$ lat. plēnus, gen. plAné-sjò in germ. fullalit. pilna- etc. = sankr. pūrnáa.$\left.-^{2}\right)$ Das part. von mara $(m r)$ sterben war mértò-s mrtésjò (von jenem sanskr. márta-sterblich). Auf $s \bar{a}^{\prime}-t \grave{o}-s$ sa-té-sjò (jenes aus $s a ́ A, a-t \grave{a}$ ) satt lässt got. $s \hat{o} b$ (vgl. lit. sôtus) neben sad-s schliessen: ebenso verhalten sich sauskr. çāta-und çitá-, lat. catus, abaktr. stāta-stehend und sanskr. sthitá-. Arézntò-m Arẓnté-sjò silber, von jenem mit regelmässigen schwund des anlautenden $A$, ausser dem accent, sanskr. razatá-, von diesem lat. argentum. Lat. hämus aus den starken, griech. $\chi \breve{\alpha} \mu o_{s}$ aus den schwachen casus. Got. stôl-s stuhl und lit. stála-s tisch sind stälò-s stalé-sjò. Lat. ācer

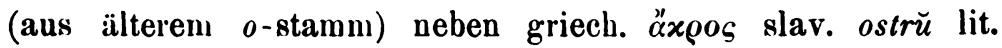
usztras spitz zeigt uns ein $\bar{a}^{\prime}$ crò-s acré-sjò. Zahlreiche accent-

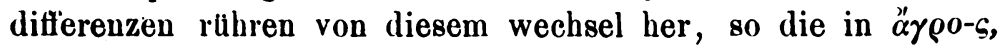
und sanskr. azrá- aus $\bar{a}_{z}^{\prime}$ rò-s azré-sjò, xv́xגo-s und iskr. cakrá(zu Verner, Kuhns zs. 23, 119). Auch accentdifferenzen zwischen der vedischen sprache und dem späteren sanskrit beruhen auf diesem accentwechsel ${ }^{3}$ ), ebenso solche zwischen den germanischen dialecten (so weist das nordfries. auf blapa neben blada blatt zurïck). Das urgermanische scheint den accentwechsel gewahrt zu haben in

1) Wenn man den anlaut $c v$ mit rein palatalem $k$ zu sprechen ver-

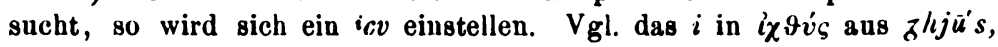
ixrivos aus $c j$ - und anderen wörtern. Auf eine anlautsgruppe weist uns auch das $x x, \pi \pi$ : im inlaut wäre einfaches $\pi$ entstanden. Die stufen-

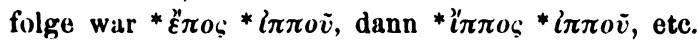

2) 'Stier' seheint zu sein stéuArj-s, gen. stuAre'sjo (uA wird $u$ ). Doch bleibt dabei das $a$ in $\tau \alpha \tilde{v} \rho o-\varsigma$ unerkliirt. Urspr. ciä vio-s ciAvé- : aus jenem skr. $\varsigma^{j a} v \dot{a}$ - dunkelbraun, aus diesem lit. szyvas slav. sivŭ aschfarbig.

3) Eine directe accentverrïckung im einzelnen worte (sie sei denn die wirkung einer analogie) hat im sanskrit, im griechischen und im germanischen überhaupt nicht stattgefunden. Der griechische accent, soweit er frei ist, ist anch wo er vom indischen und germanischen abweicht doch iiberall vertreter eines ursprünglichen accents. Etwas anderes als die abgewiesene accentverriuckung ist es, wenn in einem worte das in der grundsprache zwei accente hatte (das also ein doppeltes wort war), die jüngere sprache den einen fallen lässt. S. die anm. an schlusse dieses abschnittes von der entstehung des $o$. 
wörtern wie kunénho-z könig, abl. kunungé', woher der wechsel zwischen *-îh und -ung, dann -îg und -ing (s. Paul btr. VI, 546). Von der ursprlinglichen constanten betonung des genitivs - ésjo (oder vielmehr -éso, s. d. anm. s. 500) rührt das tonlose $s$, nicht tōnende $z$ des germanischen genitivs, an. úlfs, ebenso von der ursprünglich constanten barytonierung des nominativs $-o s$ (wie des -is, -us und $-s$ in der cons. decl., s. u.), welche die mehrzahl der wörter gewahrt haben wird, das ebenso constante germ. tönende $-z$ des nominativs, an. $-r$.

Die beiden direct aus der grundsprache stammenden formen des accentwechsels sind die uns jetzt bekannten: der accentwechsel 1. dárcà $d$ arcá, woraus dércò- drcé-, und der accentwechsel 2. dar'c,a darc, a, woraus dor'c-drc-(oder wenn der vocal der genitivendung -os \%um stamme gehört, s. s. 499 anm.2, dar'c a d arcì, woraus dor'c-drcò-). Beim accentwechsel 1. hat der nom. sing. die gestalt eines paroxytonons, écvòs svépnòs etc., beim accentwechsel 2. sind die formen der starken casus properispomena (das $-s$ des nom. sing. muss eine eigne silbe gebildet haben), nom. dor $c-s$, roùk-s, $p \bar{o}^{\prime} d-s, d j \bar{o}^{\prime} v-s$, acc. roù $k-m$, $p \bar{o}^{\prime} d-m, d j \bar{o}^{\prime} v-m, g^{u} \vec{o}^{\prime} v-m, n \bar{o}^{\prime} k t-m$, nom. plur. $p \bar{o}^{\prime} d-E s(*-E, a-s, \dot{a})$ etc. Ich will bis wir die vorgänge besser kennen und treffender bezeichnen können jenen accentwechsel den udäta- oder hochtonwechsel, diesen den svarita- oder tieftonwechsel nennen.

Der udāttawechsel ist identisch mit F. de Saussures 'flexion faible', der svaritawechsel mit seiner 'flexion forte'. Welchen wörtern aber ursprunglich 'flexion faible' zukomme (gen. - $\varepsilon j o s$,

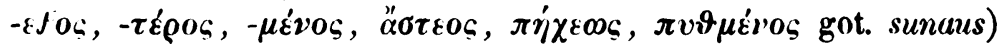

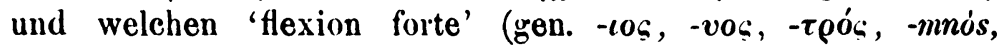

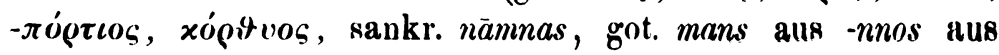
-nvos), haben wir noch nicht erfahren. 'Flexion forte' haben von haus aus die paroxytona, 'flexion forte' die properispomena der grundsprache.

Die neutra auf -os hatten als paroxytona den udāttawechsel. Also stammt die wortform griech. $\beta \dot{\alpha} \vartheta o \varsigma, \pi \alpha \dot{\vartheta} \theta \varsigma$ neben

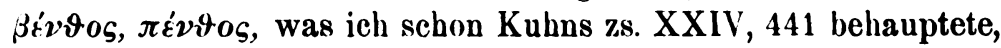

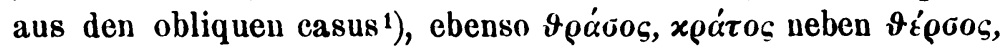

1) 'Ohne weiteres' war dies damals nicht angenommen, wie Osthoff Morph. us. II, 17 meint, vielmehr glaubte ich schon damals, dass ur- 
xózoc, doch war die ältere flexion nicht die a. a. o. vernutete (gen. z. b. *dhrsesós), sondern dhirsòs, gen. dhrsésòs. Saussure s. 129 und Osthoff, Morph. II, 17 anm., erklären $\pi \dot{\alpha} \vartheta 0$ : als

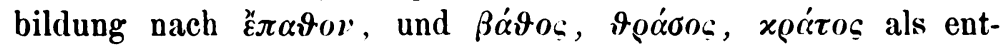

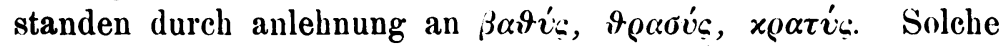
erklärungen durch 'falsche' analogie müssen zuriicktreten gegenuber einer erklärung durch den berechtigtsten und notwendizsten vorgang, die ausgleichung zwischen zwei ohne einen dem sprachgefibl erkennbaren grund nebenein ander herlaufenden doppelformen. Es giebt ausser diesen griechischen noch zahlreiche zeugen für den ursprïnglichen accentwechsel in der flexion der neutra auf -os. In sanskrit die infinitive auf -ás $\bar{e}$ (Delbrick, Ai. verb. § 202), dative unsrer neutra. Auf germanischem boden das gotische gadigis neben $\tau \varepsilon i \chi o \varsigma$, die gotischen -is, dat. -isa (rimis, agis $\mathrm{m}$.), neben -iza (rikviz, hatis), das nordische hons $=$ ahd. huonir (vorgerm. cắnos, gen. éanésos, dann cānésos: der nordische plural setzt einen sing. *hœens voraus, der wie die gotischen wörter der $a$-decl. folgte), auch in manchen aus ursprunglichen $s$-stämmen hervorgegangenen wörtern der vocal oder der auslautende consonant der wurzelsilbe. $\left.{ }^{1}\right)$ Sanskr. $\bar{a}^{\prime}$ gas n. $=$ griech. ắos war ăgòs (*éAgòs), gen. agésòs (Agés-) (so schon Joh. Schmidt, KZ. XXV, 23). Nach Saussure 168 kann das $\varepsilon$ in griech. "̈dos $=$ skr. súdas neben lat. sēdes, ebenso das $\varepsilon$ in $\tau \dot{\varepsilon} \gamma o s$ ursprianglich nur der schwachen form zustehen, wir haben also sế $d o s$ (*séEdos) sEdésòs. Zu got. agis (nasc. geworden), ae. eze m., gehörte als nom. acc. ôgs.

Im nom. acc. plur. der neutra auf -os muste der svaritierte vocal vor doppeltem anulätta gedehnt werden, ménòs (minus a), plur. mén ò'sA (mánìs $a-A, a)$. Das so entstandene -ōs haloen die zahlreichen ius masc. genus iibergetretenen latein. wörter auf $-o s,-\bar{o} r$ - verallgemeinert. Dasselbe, germ. -īz-, wird das ae. -or sein in sižr m. etc., neben ae. size as. sigi aus ségez-.

spriinglich in der flexion cines jeden wortes ein accentwechsel stattgefunden habe.

1) Neben ahd. as. href n. aus krépo-m oder krépis haben wir das lat. corpus aus krpe's-. Die wörter jugi-m und jeugos sind urspr. -jéugím jugésjo und jéugós jugisos. Daher der leichte ïbergang zwischen neutren auf $-o-m$ und $-o s$. 
Die geschlechtigen stämme baben im nom. acc. sing. dasselbe $-\bar{o} s$ (nom. $-\bar{o} s$ aus -ìs $a-s, a$, acc. $-\bar{o} s-m$ aus $-\grave{a} s, a-m, a$ ). Von Ausés-, sanskr. usás-, muste der nom. acc. mit udātta in der stammsilbe lauten $\bar{a}^{\prime} u s \overline{o s},-m\left(\bar{a}^{\prime}=\dot{e}^{\prime} A\right)$ : der lange vocal der wurzelsilbe ist erhalten in griech. joós (jvos aus * $\eta v \sigma \omega s)$, dessen accent aber aus den obliquen casus stammt.

Der nom. plur. der geschlechtigen wörter auf $-\bar{o} s$ hat die gestalt $-\bar{o} s E s$ (aus - $\grave{s}, a-E, a-s, a$ ?). Als seitenstück zu dem ubergreifen der pluralendung der neutra auf $-o s$ in den plural der neutra auf $-0-m$ haben wir das übergreifen dieser endung -oses in den nom. plur. der wörter auf -o-s, im indoiran. ved. -āsas altpers. $-\bar{a} h a$. Eine unsichere spur desselben übergriffes auf europäischem boden, vom germanischen abgesehen, könnte man im latein. gen. plur. -ōrum finden, doch konnte diese form der endung des gen. plur. auch auf anderem wege erreicht werden. Dass die ostgerm. pluralendung der $o$-stämme, got. - $\hat{s}$ an. -ar, irgend etwas anderes ist als der ursprüngliche den $o$-stämmen geblihrende plural, $-\vec{o} s$, germ. $-\bar{o} z$, kann durch nichts bewiesen werden. Áber die altfriesische pluralendung -ar, fiskar, des emsfriesischen (oder münsterfriesischen, d. i. der mundarten BEH.) und des einen der beiden nordfries. dialecte (neben $\operatorname{dem}-a$, aus $-\bar{o} z$ oder aus dem acc. $-\bar{o}^{n}$, des weser- oder bremerfries. und des andern nordfries. dialects) kann absolut nichts anderes sein als $-\bar{o} z e z$, und dieser friesischen pluralendung -ar zur gesellschaft werden die pluralendungen der nachbardialecie, altsächs. -os, altengl. -as, altes $-\bar{o}^{\prime}$ ses sein. Das as. -os ae. -as könnte auch germ. - $\bar{o}^{\prime} s$ sein (s. Paul, btr. VI, 550), da uns aber der nom. sing. der $o$-stämme constant als barytoniertes $-o z$, in keiner germ. mundart als oxytoniertes - $\delta s$ vorliegt, so wage ich nicht eine pluralendung der 0 -declination auf ein oxytoniertes $-\bar{o}^{\prime} s$ zurückzuführen. Bei den $s$-stämmen dagegen finden wir in den germanisclien dialecten ein starkes schwanken des accents, s. o. hons neben huonir, agis- neben rikviz-, es steht also der annahme eines - $\bar{o}^{\prime}$ sez neben -ōzez nichts im wege. ${ }^{1}$ ) Dass aber das indoiranische und ein teil

1) Gerade in dem teile des germ. gebiets, dem wir den plural -ozzez $\left(-\tilde{o}^{\prime} s e z\right)$ zuerkennen, finden wir die endung in dem namen des wahrscheinlich in Schleswig sitzenden volkes der Eudoses (die identificierung 
des germanischen in der verwendung der pluralendung -oses für $o$-stämme ubereinstimmen ist der reine zufall, $d$. $h$. es hat zwar wie alles einen inneren grund, aber der grund ist nicht das frühere bestehen einer gemeinindogermanischen pluralform -ōses für wörter auf -o-s.

Genau dieselbe war natürlich die flexion der comparative auf -jos (aus $-j \grave{a}-s, a$ ): rom. sing. m. f. $-j \bar{o} s$, n. $-j o s$, gen. -jésòs. $m \bar{a}^{\prime} c j \bar{o} s$, neutr. $m \bar{a}^{\prime} c j o ̀ s$, gen. macjésòs: griech. $\mu \dot{\alpha} \sigma \sigma \omega \nu$ neutr. $\mu \tilde{\alpha} \sigma \sigma o v$, comp. zu $\mu \alpha x \rho o ́-\varsigma$, abaktr. maçāo. Das germanische maiz, ae. mâa, stammt aus dem macjés-der obliquen casus, indem palatales $g$ unmittelbar vor $j$ (nicht vor -éjo-) im germanischen schwindet wie velares $g$ vor $v$. Comp. bhéuAjō's, -jòs, gen. bhuAjésòs giebt sanskr. bhävījas aus den starken, bh $\bar{u}^{\prime} j a s$ ( $\bar{u}$ aus $u A$ ) aus den schwachen casus. Der accentwechsel im superlativ war mä́cistòs, gen. macisté-sjò, griech. $\mu \dot{\eta} x \iota \sigma \tau o s$, altpers. mapista-. Ursprünglich dreisilbige wurzeln (wie dajava s. 492 , woraus daiv und djau), behaupten im positiv das erste, im comp. und superl. das zweite $a$, für welchen wechsel die regel wol noch einnal zu finden sein wird: avara 'weit' und para.4a 'viel' erscheinen im positiv als aur und $\operatorname{par} A$ (griech. $\varepsilon \dot{v} \rho v$ - und germ. felu-, s. u.), im comp. und sup. als var und praA, comp. vérjō's, *urjésòs, sankr. várījas, $\operatorname{pr}_{\bar{a}}^{\prime} j \bar{o}{ }^{\prime} s(\bar{a}=\dot{e} A)$ griech. $\pi \lambda \dot{\eta} \omega \nu, \pi \lambda \varepsilon i c o v$, gen. prajésòs (an. fleiri), sup. véristò-s ${ }^{*}$ uristé-sjò, prä́istò-s (= préAistò-s, gr. $\pi \lambda \varepsilon \tau \sigma \tau 0-\varsigma$ ) praisté-sjò (an. flestr). Sup. rézistò-s rzisté-sjò, krétistò-s krtisté-sjò: aus den starken casus sanskr. rázistha-, aus den schwachen sanskr. $r^{\prime} z^{i s t h a-~ u n d ~ g r i e c h . ~ x \rho \alpha ́ \tau \iota \sigma \tau o-s . ~}$

Denselben accentwechsel wie die paroxytona auf $-s$ hatten

des volkes mit den Jïten widerspricht natlirlich den lautgesetzen, die stammsilbe ist dieselbe wie im namen der Iâde mit fries. iâ aus eu). Wäre die pluralendung -ōses gemeingermanisch gewesen, dann müsten wir auf schritt und tritt bei den römischen schriftstellern auf -öses sich reimende germanische völkernamen finden, ${ }^{*}$ Suébóses u. s. w. Die Römer erfassten in germanischen namen entweder den germanischen nominativ oder den germanischen accusativ, und machten denselben in ihrer eigenen sprache zum accusativ oder nominativ je nachdem es für die lateinische flexion sich schickte: die zahlreichen germanischen pluralnominative auf $\bar{o} s$ (germ. $-\partial z z$ ) behandelten sie also als lateinische accusative, Suébös, und musten die volksstämme daher im nom. Suébi etc. nenueu. 
die auf $-t$, also - $e^{t}$ - in den obliquen casus, - òt im nom. acc. neutr., $-\bar{o}^{-} t$ - im nom. acc. m. f. und im nom. acc. plur. Ein neutrum dieser klasse war got. liuhad, germ. leuhado- (aus * lézıkòt, gen. *lukétòs), das die gestalt der starken casus im eu, im $h$ und im $d$ gewahrt hat. Das lat. caput hat den wurzelvocal der obliquen casus in den nom. ace. dringen lassen, ebenso das germanische wort (dessen $a u=a+u$-epenthese, bewirkt vom folgenden $p$ oder $k u$ ): das -id in got. haubip as. hôbid ahd. houpit ist das -et- der obliquen casus, das ae. -od in héafod $=$ an. *aঠ ist das $-\bar{o} t$ - des plurals, das altnord. - $\iota \delta$ in hofuð ist ${ }^{*}-a \partial u$ aus $-\bar{o} t \bar{o}$ (vgl. kolluðu). Ebenso haben die beiden folgenden wörter durchweg den wurzelvocal $a$ aus den obliquen, das $d$ aus den starken casus: ae. roced haus, hoced hecht zeigen das -ot (germ. -ada), ae. reced, ahd. hehhit das umlautwirkende -et-, as. racod, ae. hacod das - $\bar{o} t$ - des plurals. Vgl. Paul, Beitr. VI. 227 f.

Die feminina auf - $A$ mit dem vocal $e$ in der wurzelsilbe (udāttawörter $=$ paroxytona) verhalten sich zu den wörtern auf $-o$ nicht anders als die neutra auf $-s$. Sie hatten also auch den gleichen accentwechsel. Dem -òs : -és- ist analog $-\bar{o}:-\bar{a}^{\prime}-$ $\left({ }^{*} \partial A:{ }^{*} \dot{e} A\right)$. Dem -ésò- $(\dot{a} s \grave{a}-)$ des genitivs ist analog $-\hat{a}-(-\dot{a} A \grave{a})$, mit dem circumflex weil in der länge ein udāttavocal und ein svaritavocal durch contraction vereinigt sind. Da die genitivendung $-\hat{\alpha} s$ den circumflex, nicht den acut hatte, heisst es germanisch constant $-\bar{a} z$ (nicht $-\bar{a} s$ ). ${ }^{1}$ ) Es hiess also urspr. téutò', gen. *tutds: der wurzelvocal der obliquen casus ist geschwunden, das got. piuda wahrt aber den accent derselben. Dem nominativ sing. kann keine andere endung gegeben werden als $-\bar{\delta}$, téut $\bar{a}$ ist als aus der grundsprache direct hervorgegangene form unmöglich, teutā' ebenso: möglich wäre bloss noch die svaritaform tóut $A$ (s. u.). Den accentwechsel der grundsprache zeigt noch ig. guénò weib, gen. gunds: aus dem nom. slav. $\check{z} e n \bar{a}$ got. kvinô abd. chwena, aus den schwachen casus skr. gnā griech. $\gamma v v \dot{y}$ boeot. $\beta \alpha v \bar{\alpha}$ an. kona etc. Dies wort ist als ursprunglichen accentwechsel verratend schon von Osthoff, Morph.

1) Dieser circumflex in contrahierten silben traf der zeit nach nicht mehr mit dem ursprünglichen svarita zusammen, der im urgermanischen schon zum udātta geworden war. Die contractionen stammen gewiss nicht aus der grundsprache. 
unters. II, $13 \mathrm{ff}$. angefuhrt (vgl. Joh. Schmidt, KZ. XXV. 129). Neben lat. nāsus m. und sankr. $n \bar{a} s \bar{a}$ f. haben wir slav. nosü m., ahd. nasu (germ. násō f.): zu grunde liegt ein masc. $n \bar{a}^{\prime} s \grave{o}-s$, gen. nasésjò, fem. $n \vec{a}^{\prime} s \bar{o}^{\prime}$, gen. nas $\hat{s}$ (das $\bar{a}^{\prime}=\dot{e} A$ ). ${ }^{1}$ ) Ebenso verhalten sich langobard. fereha aesculus (knérk $u^{\prime}$, vgl. lat. quercus) und ahd. foraha (mit den accent der starken casus) (gen. kurkuâs). Ahd. liupa ae. lufu liebe ist léubhò' g. lubhâs. Den

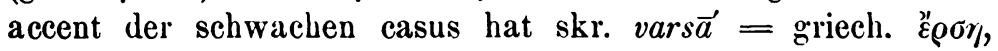
aus vérs $\bar{o}^{\prime}$, g. vrs $\left.\hat{a} s^{2}{ }^{2}\right)$ Die feminina auf $-\bar{a}$ - erscheinen nach Saussure 233 in zwei gestalten, 1) als oxytona mit reducierter wurzel, 2) als paroxytona mit dem vocal $e$ : jene haben die form der schwachen, diese die der starken casus verallgemeinert. Feminina zu den in der grundsprache dreisilbigen masculinen wie suépnò- schlaf sind griech. $\tau \dot{\varepsilon} \chi v \eta$, germ. érpo erde. ${ }^{3}$ )

Bei allen suffixen haben wir svaritawörter (properispomena im nom. acc.) neben den udāttawörtern. So würden wir von ruk (rauka) 'leuchten' haben (mit europäischem $l$ )

ud. léukò- léukòs léukòt léukòn léukmön- léuktōr $r$ svar. löuk- löuks- louikt- loükn löukmn louktr.

Udāttawort ist an. liómi, svaritawort lat. lümen, f. got. lauhmuni.

Mit dem suffix $-s(s, a)$ gebildet ist got. bariz- (aus * bhäròs bharésòs $)$ das udāttawort, lat. farr- (*bhōrs bharsòs) das svaritawort, ebenso verhält sich zu lat. acus -eris, abd. ehir (aus * $\bar{a}^{\prime}$ còs acésòs) das got. ahs (aus ${ }^{*} \bar{o} c s$ acsòs), zu sanskr. ájas (aus ${ }^{*} \bar{a}^{\prime} j o s$ ajésòs) das lat. ues, got. aiz (aus *'ंis aisòs). Udāttawort ist * $\bar{a}^{\prime} i v \bar{o} ' s$ aivésòs (griech. acc. aĩ̄, vgl. Joh. Schmidt, K. z. XXV, 25), svaritawort $\bar{o}^{\prime} j u s$ (skr. $\bar{a}^{\prime} j u s$ ) *ajusòs.

Die wörter auf $-o,-t o,-n o,-m o,-r o,-s o$ etc. mit dem vocal $o$ in der stammsilbe, wie sie besonders im griechischen

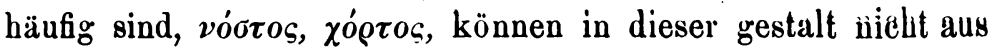
der grundsprache stammen. Die grundsprache müste sonst wörter mit doppeltem svarita, dem einen auf der stammsilbe, dem andern auf dem suffix, und dazwischen stehendem anudātta besessen haben (*iàs,a-tà). Diese wörter können nur von svarita-

1) Urspr. $\bar{a}^{\prime} c \vec{o}$ spitze, schärfe, gen. acâs: ion. ’’ $x \dot{\eta}$ neben $\stackrel{2}{\alpha} \dot{\eta}$. Ebenso hiess es urspr. $\vec{a} c v \vec{v}$ wasser, gen. acvâs (got. ahva hat den accent der starken casus), $\vec{a}^{\prime} c s \vec{o}^{\prime}$ (mhd. uohse achselhöhle), gen. acsàs.

2) Ganz ebenso got. fairzna ferse mit $z$ neben sonstigem germ. $s$.

3) Germ. wol lépiō, gen. lupiäz, der fluss Lippe. 
wörtern stammen, also aus consonantischen stämmen von der art des ziemlich vereinzelt ibrig gebliebenen nokt-nacht, die $o$-stämme geworden sind durch den allergewöhnlichsten vorgang, den übertritt aus der consonantischen in die $o$-declination. Das nordeuropäische snoigh $u_{0-s}$ schnee ist hervorgegangen aus älterem *snoighu-s, gen. suighuòs (gr. vıcós lat. nivis). Griech. доıлós gern. laiba- muss entstanden sein aus einem * roiku-s, gen. rikuòs, das enthalten ist im svaritapräsens roiku-mi (lit. lëku, alt lëkmi) nehen dem udāttapräsens réiku $\left.\bar{o}^{\prime}(\lambda \varepsilon i \pi \omega) .{ }^{1}\right)$ Germ. stauba- (ahd. stoup dän. stov) und stuba- (nnd. stof) ist ${ }^{*}$ stoùbh-s, *stubhòs, ebenso verhalten sich hlaut-s und hlot u. a., germ. dauba- taub ist *dhoùbh-s*dhubhòs. Got. ga-nôh-s altfries. $\hat{e}$-nòch etc. zum verb got. ga-nah prät. *nauhta part. nauhts ist herrorgegangen aus consonantischem $n \bar{o}^{\prime} c-s$, gen. ncòs: das nordfriesische $\hat{\imath}$-noch mit sicher ursprünglich kurzem 0 (vgl. got. ganauha m.), also ein germ. nuga- neben nogga-, kann nur aus dem schwachen stamme urspr. nc- hervorgegangen sein. Lit. dva'ras slav. dvorŭ ist dem consonantischen $d h v \bar{o} r-s$ dhuròs entsprossen, ebenso sind die griechischen $x \lambda o \pi \dot{o}-\varsigma$ dieb, $\sigma x o \pi \dot{o}-\varsigma$

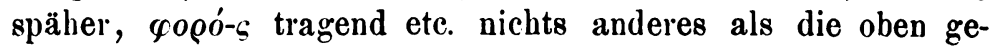
sehenen $x \lambda$ ó $\psi$ etc., hervorgegangen aus den schwachen stämmen ${ }^{*} \sigma x \varepsilon \pi-,{ }^{*} x \lambda \alpha \pi-$, dann ${ }^{*} \sigma x o \pi-, x \lambda o \pi-$. Sanskr. bhāra- ist dasselbe aus dem starken stamme: ebenso verbalten sich zu einander alle indoiran. $\bar{a}$ und griechischen $o$ in wörtern wie skr. srāva-, gr.

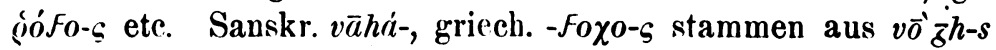
$u \bar{z} h \grave{s}$, das im indischen noch consonantisch und ablautend erhalten ist: nom. $-v \bar{a}^{\prime} t$ acc. $-v \bar{a}^{\prime} h a m$, anad-vă $\bar{a}^{\prime} h$-ochse, gen. anaduhas. Das griech. $-\dot{\infty} \pi 0-\epsilon$ in $\alpha \nu \vartheta \rho \omega \pi \circ \subseteq$ (uber welches 8. Bezzenberger in seinen Beitr. $\mathrm{V}, 168)=$ slav. $-\bar{a} k \breve{u}$ ist älteres consonantisches $\omega \dot{\pi}$ - $\left.\dot{j} \boldsymbol{\pi}-.^{2}\right)$ - Ebenso bei abgeleiteten stämmen. Die -to- mit vorhergehendem vocal $\dot{o}$ sind hervorgegangen aus

1) Griech. Foixoo-c und sanskr. veç̧á-, wenn dieses wie Saussure s. $\$ 3$ annimmt $o i$ hat, sind aus dem cons. stamme voic-s vicos erwachsen.

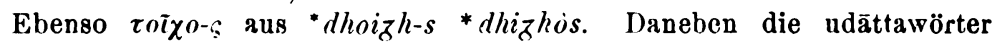
veico-s, lat. vicus, und dheizho-s, abaktr. daeza-osk. feiho-ae. dic.

2) Aus der starken stufe eines urspr. söm-s smós stammt abaktr. häma- slav. sāmŭ ae. że-sìm, aus der schwachen wit geändertem ablautsverhältnis (wie im jüngeren part. svaran-s mbd. geswarn zum perf. svôr, swuor, neben dem älteren part. sworen mhd. gesworn) das gleich- 
stämmen wie nókt-, ibber dessen entstehung s. o. Slav. zolto gold (abulg. zlāto) ist urspriinglich cousonantisch * zhor'At (grundf. Jhar' $A$ at a) mit dem vocal des starken stammes, das germ. gúlba- dagegen ist aus dem schwachen stamme desselben wortes, $\zeta h A_{l-}$, erwachsen. Germ. fahsa- stammt aus consonantischem pocs-, das entstanden ist wie nokt-, aus * ${ }^{*} \bar{o}^{\prime} c s$, $p$ csòs, dem svaritawort zum udāttawort pécòs. Germ. hálsahals ist ${ }^{*}$ cor'As, gen. * crAsòs. Germ. vōkra- wucher hat das lange $\bar{o}$ des starken stammes bewahrt, es ist ein $v \bar{o}^{-} \zeta r$, gen. *uzròs. Westgerm. watra- wasser ist, wie jeder weiss, aus der consonantischen in die $o$-decl. übergetreten, es war $v \bar{o} d r$ (grundform vàd $a-r a$ ), gen. udnòs, lann fürs westgerm. *udròs (und mit übergang des ablauts $v \bar{o}: u$ in $v \bar{o}: v o$ ) vodros. Griech. oैvvx- ist n'ghu- (Fick, Bezz. V, 167), wozu als starker stamm $n \bar{o} g h u$ - gehörte, das entsprechende germ. wort, an. nagl, ist ${ }^{*} n \bar{o} g h r-s$ gen. ${ }^{*}$ gh hròs, dann noghros: an diesem worte lässt sich noch erweisen, dass es früher der consonant. declination angehörte, denn seine consonant. declination ist im nordischen bewahrt, plur. an. negl (aus germ. náglez). ${ }^{1}$ ) Wie auf $-r(-l)$ und $-n$ so gab es auch svaritawörter auf $-m$, die dann in die $o$-decl. ubertraten, -mo. Unser germ. arma-z brachium stimmt in der wurzelsilbe nicht zu lat. armus = sankr. ìrmá- (s. Saussure s. 263): diese stammen aus den schwachen casus, gen. ${ }^{*}$ rAmòs, jenes aus den starken, nom. ${ }^{*}$ or' $A m-s$, dann orAmo-s. Ebenso verhalten sich ahd. toum rauch und lat. fümus skr. dhüma- aus *dhoùAm-s, gen. dhuAmòs ( $u A$ wird $\bar{u}$ ). Doch können die latein. und indischen wörter auch urspr. udāttawōrter sein, aus dem -mé- der schwachen casus ent-

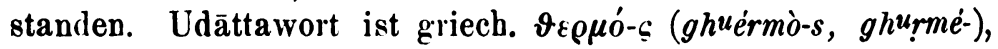
lat. formus kann aus dessen schwachen casus erwachsen sein,

bedeutende got. sama griech. ó $\mu \dot{o}-\varsigma$ abaktr. hama-sanskr. samá-. Ebenso entstand aus $d \grave{o}^{\prime} m$ (griech. $\delta \tilde{\omega}$ ), gen. $d m \dot{s} s$ das griech. $\delta o ́ \mu o-\varsigma$ sanskr. damá-. Jenes $s \vec{o} m$-s (grundf. sàm,a) bedentet 'éin', 'ein und derselbe': aus dem schwachen stamme $s m$ - ist ferner mit der abgeschwächten bedeutung 'ein' (unbestimmt) das wort sanskr. sama- gr. $\alpha \mu o-$ got. sum-s ae. sum erwachsen. Der schwache stamm $s m$ - ist in der bedeutung 'éin' noch vorhanden als erstes glied von compositen, sankr. sa- griech. $\alpha$-, $\dot{\alpha}-\delta \varepsilon \lambda \varphi \dot{o}^{-}-\varsigma\left(s m-g^{u} e ́ r b h \dot{o}-s s m-g^{u} r b h e ́-s j o\right)$ etc.

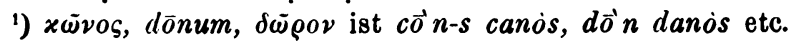


aher germ. varma-z und das subst. skr. gharmá- sind urspring-

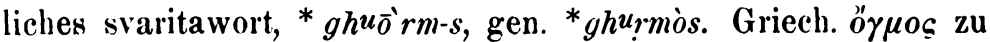

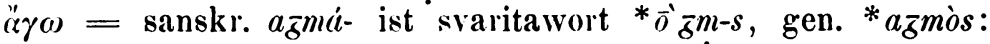
das grieck. 0 entsiand durch den iibergang des ablauts $\ddot{o}: a$ in $\bar{o}: 0$.

Die feminina auf $-\bar{a}$ - mit dem wurzelvocal $o$ sind entweder, analog den eben gesehenen masculinen und neutren, aus weiblichen consonantischen stämmen hervorgegangen, wie $\mathrm{zb}$. wol ahd. zêha an. $t a \dot{~ f}$. aus dem cons. stanme germ. taih-, plur. an. 10 r aus táihez, oder es sind alte svaritawörter auf $-A$. Also z. b. griech. $x \dot{c}^{\prime} \pi \eta$ ist entweder ein fem. ${ }^{*} c \bar{o}^{\prime} p-s$, gen. ${ }^{*} c a p \grave{s}$, oder ein fem. ${ }^{*} c \bar{o}^{\prime} p A$, gen. ${ }^{*}$ capÀs. Solche svaritawörter auf - $A$ sind die feminina auf $-i A$, griech. $\cdot \omega \alpha$ mit dem accent der starken, sankr. $-\bar{\imath}^{\prime}$ nit dem der schwachen casus. Urspr. ${ }^{*} \bar{o}^{\prime} \operatorname{tni} A$, gen. ${ }^{*} p$ tniAiss, sanskr. mit der form der schwachen casus patní, griech. mit dem $o$ des geänderten ablautverhältnisses (wie in $\pi o \delta-) \pi o ́ \tau v \iota \alpha$. 'Geberin' ist $d \bar{o}^{\prime} \operatorname{tri} A\left(\bar{o}^{\prime}=o A\right)$, datriÀs: hier hat skr. dātrí den vocal der starken casus, ein lat. datri-c $c$ - den der schwachen. ${ }^{1}$ )

Bei den $i$ - und $u$-stämmen und den $r$ - und $n$-stämmen betrachten wir zunächst nur die reine svaritaflexion und die reine udāttaflexion der grundsprache, um darauf erst die jüngeren umgestaltungen ins auge $\mathrm{zu}$ fassen.

$i$ - und $u$-stämme. Svaritaflexion ist $\bar{\delta}^{-} n u$ knie (grundf. żin av,a), gen. ẓ̇uòs, dō'ru bolz, gen. druòs, plur. dō'ruA $\left(d a^{\prime} r a v a-A a\right)$. Ein griechisches ursprüngliches prosperispomenon

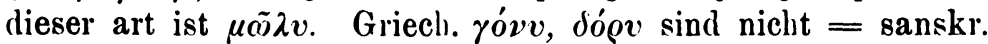

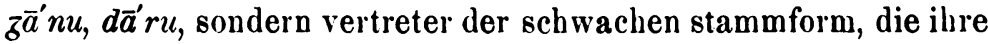
gestalt gewannen durch änderung des ablauts $\infty:$ - in $\omega: 0$ (s. Joh. Schmidt, Kuhns zs. XXV, 50 f.). Lat. mari- mare ist ursprüglich schwache stammform, die frühere flexion war $m \bar{o} r i\left(\bar{o}=o^{A}\right)$, gen. mariòs (vgl. ae. môr ahd. muor palus). Das lange $\bar{o}$ des ursprtinglichen properispomenons sehen wir in ne. woof einschlag beim weben, d. i. urspr. vōbhi n., gen. ${ }^{*} u b h i o s$ (das wort heisst nordfriesisch $\hat{e} f$ mit schwund des $w$ vor $\bar{o}$ und $i$-umlaut).

1) Wir finden in wirklichkeit nicht den gen. -os sondern den gen. - âs nach der analogie der wörter auf -oA. Griech. $\mu i \alpha$ gen. $\mu \iota \tilde{\alpha} \varsigma$ ist das fem. zum oben angesetzten $s \vec{o}^{\prime} m-s$ smòs, also ${ }^{*} s \vec{o}^{\prime} m i \Delta$, gen. ${ }^{*} s m i \Delta \dot{o}$, dann smiâs. 
Verschiedene svaritaformen auf $-i$ gelten als nom. acc. zu schwachen casus mit $-n-$, so cor'di herz, gen. crdnòs, pò uni feuer (got. fôn), gen. *punnios (uber dieses wort s. u.). Geschlechtige stämme: on'su-s, gen. nsuòs, an. áss, sanskr. ásu-. or'bhu-s, gen. ?’bhù̀s, sanskr. rbhú- (das $o$ im germ. worte an. álfr alıd. alp). Slav. loš̆ ist or ci-s, ${ }^{*}$ rciòs (vgl. oben ércò-s rcé-sjò, griech. ${ }^{\prime \prime} \lambda x \eta$ ist *ércō $r c \hat{a} s$, lat. alces ist ein fremdwort). Got. sauls, ahd. sûl ist soìA-li-s suAliòs. Adj. $\mu \tilde{o} \lambda v-\varsigma-v o c$, fem. lat. mōli- (nom. mōles), von mara (mr) zerreiben, ist $m \bar{o}{ }^{\prime} r u-s$ *mruòs, mō'ri-s mriòs: die wurzelsilbe des schwachen stammes sehen wir im lat. moles-tus aus mlés-. Adj. $\bar{o} c u s(\bar{o}=o A)$, gen. *acuòs: griech. cóxíc skr. ācú- haben den accent der schwachen casus. ${ }^{*} \bar{o}^{\prime}$ cri-s spitze, fels, berg, gen. acriòs: sanskr. ácri- und griech. $\stackrel{\alpha}{\alpha} x \varrho \iota-\varsigma-\iota o \varsigma$ haben den urspr. schwachen stanm mit dem accent der starken, griech. öx@- $\varsigma$ hat dasselbe $o(: \infty)$

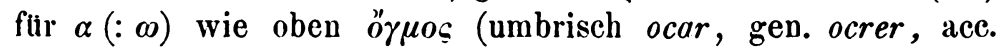
ocrem, lat. ocris haben dasselbe $o$ des geänderten ablauts). Svaritawörter, mit gen. urspr. -iòs, sanskr. -jas (und -jus, uber welches J. Wackernagel, Kuhns zs. XXV, 288. ff), griech. *p tiòs, woraus griech. $\pi \delta$ ó nom. $\pi \dot{\sigma} \sigma \iota-s$, got. fadi- (aus dem das slav. -podı́ entlehnt ist) mit demselben $o$ und vielleicht mit dem $d$ aus den schwachen casus, sanskr. nom. páti-s mit dem vocal der schwachen, dem accent der starken casus; $*^{\prime}{ }^{\prime} v i-s$ schaf $(\bar{o}=o A)$, gen. *aviòs $\left.{ }^{1}\right)$, sanskr. wie eben ávi-s, gen. ávjas, gr. öıs, oís gen. oiós, lat. ovis

1) Oder wenn Saussure mit seinem $o$ recht haben sollte, $\vec{o} v i$-s $(\bar{o}$ $=o q)$, gen. ovios, ${ }^{*} p \vec{o}^{\prime} t i-s$ gen. potios. Wie unterschieden sich in der grundsprache die wörter ovi-s schaf und avi-s vogel? Hatte jenes $\emptyset$, dieses $A$, oder war jenes properispomenon, dieses paroxytonon? Als properispomenon wäre auch dieses $* \vec{o} v i$-s vogel, gen. Avios. Das den starken casus dieses wortes beigelegte $\bar{o}$ bezeugt das $o$ für $\alpha$ in griech. oi $\omega \nu o$ 's. Wir sehen das $\bar{o}$ in dem gewiss verwandten worte 'ei', das ursprünglich ein neutrum auf $-i$ gewesen zu sein scheint, $\vec{o} v i$, gen. Avios. $\vec{o}^{\prime} v i$ ging in die $o$-decl. über ebenso wie die s. $509 \mathrm{f}$. gesehenen svaritawörter: $\bar{o} v j o-m$, griech. $\omega^{\prime} o \dot{v} v$ slav. $j a j e$, im germanischen mit zugrundelegung des schwachen stammes *avjo-m und als $s$-stamm *avjos, dann aijo-m, aijos. Im lateinischen wäre der $i$-stamm $\vec{o} v i$ ohne weiteres mit aufgebung des $i$ in die $o$-decl. iibergetreten, $\bar{o} v u m$ : das vulgärlat. ovum mit kurzem vocal, auf das sämtliche roman. mundarten zurïckweisen, wird die vocalkiirze der schwachen casus gewahrt baben. 
mit $o: \bar{o}$ für $a: \bar{o}$. Sicher hat ein solches $o$ fur $a$ durch ablautwechsel lat. scobi-s, scob-s zu scabo, also aus *skībhi-s, gen. skabhiò (oder consonantisch ${ }^{*} s k i ̄ b h-s$, skabhòs). Got. paursus diirr mit $s$ statt eines erwarteten $z$ hat den accent der starken casus gewahrt, nom. urspr. cor' $^{\prime}$ su-s. Das $o$ der starken casus der svaritawörter zeigen uns zahlreiche wörter auf $-i$ und $-u$, germ. balgi-, handu-, dies aus contú-s mit dem accent der scb wachen casus, älter coritu-s, germ. haidu- $=$ skr. kêtu- cbenso aus $k o i t u$-s aus skoitu-s, und wörter auf $-t i$ und $-t u$, wie griech. inschr. $\delta \tilde{o} \tau \iota-\varsigma=\delta \dot{\sigma} \sigma-\subseteq$ aus $d \bar{o}^{\prime}-t i \cdot s$, gen. da-tiòs. $\left.{ }^{1}\right)$

Udāttaflexion ist, der der neutra auf -os entsprechend, żenòu (grundf. żaniua), gen. żnévos. Jenes haben wir rein bewahrt in lat. gen $\bar{u}$ mit seinem langen $\bar{u}$, die gestalt der obliquen casus im germanischen, mit tibertritt in die o-decl. genau so wie bei den neutren auf $-s,-t$, kneva-, treva. Auf germ. Jélau 'viel', reine udāttaform, weist zurück das ae. fela, feola, und in den übrigen dialecten das bei svaritaflexion unerklärliche $e(i)$ der stammsilbe: die ursprüngliche flexion war pérAòu (aus párAàva), gen. prAévòs, die schwache gestalt der stammsilbe ist verallgemeinert in sanskr. purú griech. $\pi 0 \lambda v^{\prime}$ fries. ${ }^{*}$ fulu (Brokm. Ems. fule). Das fries. fiâ n. vieh kann lautgesetzlich nur aus germ. féhau entstanden sein (die fries. form könnte freilich, wenn sie es müste, aus dem jungen gen. féhauz in den nom. gedrungen sein), das germ. $h$ weist auf ein urspr. paroxytonon, also eine udāttaform, pécòu, gen. p, cévòs. Alle paroxytona mit dem vocal $\dot{e}$ müssen diese flexion gehabt haben, also médhòu n. met, deun ein pécu, médhu ist nach den accentgesetzen der grundsprache unnöglicb. Sanskr. vāsh und daneben griech. $f^{\prime \alpha} \sigma \tau \tau,-\varepsilon o \subseteq$ ist ein urspr. paroxytonon * $v \bar{a}^{\prime}$ stou, gen. vastévòs

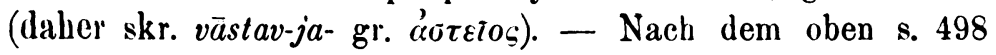
gesehenen gesetz uber die behandlung des sraritierten rocals muss das $-o i$, -ou der udāttawörter im nom. acc. plur. neutr. und im nom. acc. masc. und fem. $-\bar{o} i-,-\bar{o} u$ - werden, wie das -os zu $-\bar{o} s$ bei den $s$-stämmen. Die indogerm. form war also nicht

1) Ahd. nuohturn niichtern ist ein ursprünglich svaritiertes adj. auf -ni, nöktrni-s, gen. nktrnió-s: im germanischen besteht das wort in zwei verschiedenen gestalten, als *nōhturni-, jiinger -nia (mhd. nüehtern) aus den starken und als " "hturni- (woraus uhtern, im friesisch-englischen, dazu ahd. uohternîn) aus den schwachen casus. 
nécu-s v'́xvc, acc. nécu-m, sondern nécò $u-s$ acc. nécō' $u-m, b h \bar{a}^{\prime} \bar{c} \bar{o} u-s$ $\pi \tilde{\eta} \chi v \varsigma$, acc. $b h \bar{a}^{\prime} z h \bar{o}^{\prime} u m$, plur. nom. néc $\bar{o}^{\prime} v E s, b h \bar{a}^{\prime} \bar{\delta} \bar{o}^{\prime} v E s$. Diese flexion ist im iranischen erhalten, altbaktr. nom. sing. $b \bar{a} z \bar{a} u-s$, acc. naçāu-m, nom. acc. plur. naçāvo, dan'hāvo altpers. nom. sing. dahjōu-s, acc. $-\bar{a} u m$, nom. acc. pl. - $\bar{a} v a$. In den ubbrigen dialecten ist dieses $-\bar{o} u$ - aufgegeben, die hierhergehörigen wörter laben im nom. acc. sing. das $-u$ der svaritawörter, griech. $\pi \tilde{r} \chi v-\varsigma$ (sanskr. bāhhi- mit dem accent der obliquen casus), an. hjor-r $=$ sanskr. cáru-, an. ljor

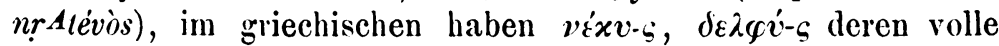
flexion angenommen, gen.-vos. An. fjord-r mit dem vocal $e$, lat. portu-s mit der stammsilbe der obliquen casus waren urspr. pértō' $u-s$ prtévòs. Got. baurnu-s 'dorn', aus den obliquen casus, ist urspr. térnōu-s trnèvòs, den vocal $e$ der starken casus hat das dän. ljorn bewahrt. Ebenso got. kvairrus an. kvirr: md. kürre an. kyrr. Das griech. verrät für das adj. ¿̇ं's 'gut' frühere udāttaflexion (s. u.), urspr. ésōu-s neutr. ésòu, gen. sévòs. Ferner war nach dem griechischen udāttawort in der grundsprache das wort 'sohn', durch (-nu und $-j u$, d. i.) na-va und $j a-v a$ abgeleitet von der wurzel sauAa $(s \bar{u})$, also nom. séuAno' $u-s$, séuAjò u-s, gen. suAnévòs, suAjèvòs, gen. griech. vícos, gen. plur.

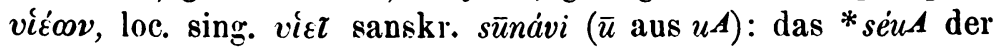
starken casus entspricht genau dem sanskr. sávi- in savi-tár-. Die griech. wörter auf $-v-$, bei welchen (nach dem gen. $-\dot{\varepsilon} f \circ \varsigma$ ) der acc. $-\dot{\varepsilon} F \alpha$ vorkommt, waren udāttawörter, also $\dot{r} \delta \dot{i}-\varsigma$, ace.

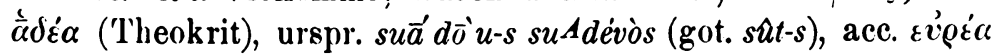
(Hom.), also éurōu-s, gen. urévòs (sanskr. urú-). - Bei den $i$-stämmen haben wir entsprechend acc. sing. $-\bar{o} i-m$, nom. plur. $-\bar{o} j-E s$, abaktr. hu-sayāi-m (a us su-sekhhòim), nom. plur. -ājo, sanskl. ace. sing. sákhājum, dual sákhājā, nom. plur. sákhājas. Die schwachen casus haben im sanskrit svaritaflexion angenommen, dat. sákhiē (statt urspr. $s, k h e j j$-). Im griechischen haben die obliquen casus mit änderung des verhältnisses $\varepsilon: \infty$ in $o: \omega-\grave{o} j$ - für -éj- angenommen, das dann auch in den acc. drang, gen. sing. $\Lambda \eta \tau o \tilde{v}_{\varsigma}$ (-ójos), lok. $\Lambda \eta \tau o \tau$, acc. $\Lambda \eta \tau \tilde{\omega}$ (-ója). Der nom. sing. der $i$-stämme hat nicht das erwartete $-\bar{o}^{\prime} i$-s,

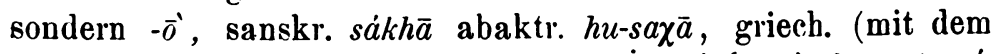
accent aus den obliquen casus) masc. $M \dot{\eta} \tau \rho \omega^{\prime}$, ' $H \rho \omega$, fem. $A \eta \tau \omega$,

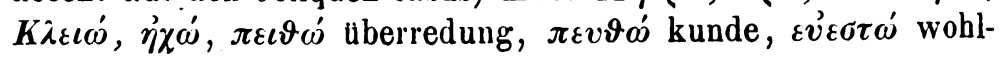


sein. Dasselbe $-\ddot{o}^{\prime}$ finden wir im nom. sing. bei den udātta-nstämmen. Lautliche erklärung des $-\bar{o}$, hier aus $-\bar{o} i-s$, ist völlig unmöglich, dort aus -ōn-s nach den bis jetzt bekannten gesetzen nicht möglich, es kann also, wenigstens für jetzt, nichts anderes angenommen werden, als dass wie mehrere $n$-stämme ihren nom. sing. auf $-i$ oder $-r$, so diese $i$ - und $n$-stämme ihren nom. sing. auf $-A$ bilden, sékhò aus sékliòd. Das $-A$ war naturlich ursprunglich nichts speciell dem feminin zukommendes, es giebt ja im indogerm. auch masculina der $A$-decl. Das - $\bar{o}$ des nom. sing. haben wir auch auf germanischem boden, im nom. sing. der altnordischen feminine wie z. b. sótt. Dass ursprungliche svaritawörter wie germ. suhti-z im nordischen obne weiteres ihre endung $-i z$ durch das $-\bar{o}$ der feminina der $\bar{a}$-decl. ersetzen konnten ist undenkbar, dieses $-\bar{o}$ wird also von den udāttawörtern stammen, denen es von anfang an zukam, und wird sich von diesen auf die feminina auf urspr. - $i$-s ausgedehnt haben. $\mathrm{Zu}$ beachten ist dass nauðr, das einzige feminin das in der nordischen prosa das $-r$ aus -iz bewahrte, ein svaritawort ist, ebenso sind die wörter, die durch den umlaut auf früheres -iz zurlickweisen, satt, att, boen (über kvan s. u.) ursprüngliche -i-s. Wie im nordischen so werden auch in den tibrigen gernıanischen dialecten manche feminine udāttawörter auf $-i$ mit dem nom. sing. auf $-\bar{o}$ nach diesem nom. einfach in die $\bar{a}$-declination übergetreten sein. Für masculina lag der übertritt in die $n$-declination am nächsten. Der name der Friesen, schwankend (auch in den fries. dialecten selbst) zwischen langem und kurzem $i$, $i$-declination und $n$-declination (die Römer hörten einen plural der $i$-decl.) ist zurluckzufuhren auf ein urgerm. Fréisō, gen. Frizéjoz. Das wort 'rocken', im nordischen und englischen der $i$-decl., im friesischen und deutschen der $n$-decl. folgend war wol urgerm. réugūo, ruguéjoz. Amelung stellt an. skagi zu gr. xүxis, zu grunde läge, wenn die zusammenstellung richtig, ein $s k \bar{a}^{\prime} k \bar{o}^{\prime}$ skakéjòs. Ahd. hîwo, lat. cîvis aus céivō civéjòs. Nach besonderheiten der griechischen flexion war das fem. $\pi \partial_{\lambda \iota \varsigma}=$ sanskr. puri- ein altes udāttawort (von derselben wurzel von welcher $\pi \circ \lambda \dot{v}$, sanskr. purú), ursprtinglich also nom. pérAó, gen. prAéjòs ( $\pi 0 \lambda \varepsilon j 0 \varsigma)$. Ein udāttawort war unser 'brust', das neutrum an. brjöst ae. bréost afr. briast as. plur. briost ein urspr. bhréustò-m bhrusté-, das fem. auf $-i$ ein urspr. bhréustō bhrustéjòs. 
$r$ - und $n$-stämme. Svaritaflexion oder 'flexion forte'. Die formen der starken casus sind properispomena. ${ }^{*} v \bar{o} d r$, gen. $u d n \grave{o}$, plur. ${ }^{*} v \vec{o} d r A$. In germanischen ist der ablaut $v \bar{o}: u$ durch $v \bar{o}: v o$ ersetzt, und dann das $v \bar{o}$ durch das vo verdrängt, plur. (nach der o-decl.) ostgerm. votno, westgerm. votrō. ${ }^{*} v \bar{n}^{-} \bar{\delta} r$ (*uznòs?), germ. vōlro- wucher, s. o. s. 510. Hierher gehören die indischen neutralformen auf $-t r$, urspr. $d \vec{o} t r$, gen. dAtròs, $d h \bar{o}^{\prime} t r$,

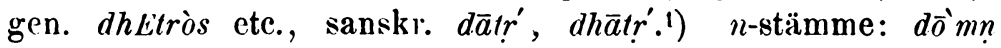

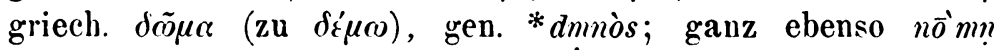

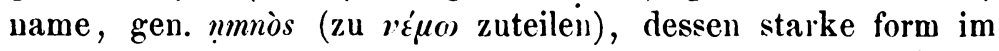
sanskrit und im latein erhalten ist, wäbrend im griech. oैvopa und im germanischen (plur. nomn̄̄) der ablaut $\bar{o}:-$ durch $\bar{o}: o$ ersetzt ist (das lange $\bar{o}$ noch in verb nl. noemen nennen). Ebenso ist griech. $\delta \dot{o}^{\prime} \mu \alpha$ entstanden aus $* d \bar{i} c m n$. Das lange $\bar{o}$ einer bildung auf $-m n$ sehen wir in $b h \ddot{a}^{\prime} r m a$ (RV.) aus $b h \bar{o}^{\prime} r m !$ (zu bhérō'). Die formen mit germ. au und $\bar{u}$ des wortes 'gaumen' (die nit $\bar{u}$ im fries. und dän.) geben zusammen ein urspr. neutrum żhoùAmn, gen. ఫ̆lıümnòs ( $\bar{u}$ aus $u A$ ). ${ }^{2}$ ) Ursprilinglich muss es zu diesen neutren auf $-\underline{r}-\underline{n}$ auch masculine und feminine gegeben haben auf $-r-s,-n-s$, so gut wie es solche auf $-i-s,-u-s$ giebt. Wo diese geschlechtigen wörter abgeblieben sind haben wir s. $509 \mathrm{f}$. gesehen, sie sind in die $o$-decl. ubergetreten. Das nordische wahrt, wie wir sahen, noch einen hierbergehörigen plural des masc. in negl. Ein hierhergehöriges adj. scheint got. baitr-s, mit oi gegenuber nordischem und westgerm. $i$, gewesen zu sein, urspr. bhoidr-s, neutr. bhoidr, gen. bhidròs. ${ }^{3}$ )

Udātaflexion, 'flexion faible'. Die formen des nom. sing. mussen paroxytona sein. Nom. acc. sing. neutr. müssen auf -òr, -òn ausgehen. Im nom. acc. plur. neutr. und im ace. sing., nom. acc. du. und plur. masc. und fem. muss nach der

1) Im RV. nur sthātur n., kein -tr. -ur ist regelrechte vertretung von auslautendem $-r$, s. J. Wackernagel Kuhns zs. XXV, $25 \tau \mathrm{f}$.

2) Die formen mit $\bar{o}$ miissen aus der einfachen wurzel zhava (neben dem erweiterten zhavAa) stammen, an. gómr aus "zht um-s, gen. * zhumos.

$\left.{ }^{3}\right)$ Die endung $-n-s$ neutr. $-n$ ist bewahrt in griech. $\mu \dot{\varepsilon} \gamma \alpha \varsigma, \mu \dot{\varepsilon}^{\prime} \gamma \alpha$ (vgl. Brugman, Morph. unters. II, $1 \dot{7} 5$ ), das aber ein udāttawort ist (s. u.), und also seine endung nach andern verloren gegangenen svaritawörtern bekam ebenso wie $\pi o \lambda \dot{v}-\varsigma \pi o \lambda \dot{v}$. 
gegrebenen regel der svaritierte vocal gedehnt werden, $-\bar{\sigma} r$, $-\bar{o}^{-} n$-. In den schwachen casus haben wir -ér-, -én- nit vorhergebendem anudātta. Der nom. singr. der geschlechtigen $n$-stïmme hat die endung $-\bar{o}$, von welchem $-\bar{o}$ wir gar nicht cinmal sagen können ob ein $n$, durch lautlichen wandel unsern blicken entzogen, darin steckt, wir müssen also dies $-\bar{o}$, wie schon oben gesagt, erklären als $A$-form zu der $n$-form der ubrigen casus. Dasselbe $-\bar{o}$ haben wir dem nom. sing. der geschlechtigen $r$-stämme beizulegen, denn ein gesetz nach welchem $-\bar{o}$ aus $-\bar{o} r$ und dies aus $-\bar{o} r-s$ (oder $-\bar{e},-\bar{e} r$ aus $-\bar{e} r s$ oder $-e r-s$ ) entstanden sein kann ist uns ebenfalls nicht bekannt. In den uns vorliegenden indogerm. dialecten ist vielfach das $-n$ und $-r$ (wie griech. das $i$ in $\Lambda \eta \tau \varphi^{\prime}$ etc.) aus den übrigen casus auch in den nom. sing. eingedrungen. Auch wenn im veda zuweilen $-\bar{a} r$ statt $-\bar{a}$ gelesen werden muss (Grassmann wb. s. VII), $h \bar{o}^{\prime} t \bar{a}-r, m \bar{a} t \bar{a}^{\prime}-r$, so ist dies nichts anderes als dasselbe eindringen des $r$ in den nominativ. - Wir haben also als gemeinindogermanisch anzusetzen die flexion des masc. nom. zhémō', gen. zh ménòs, acc. zhémō'nm, plur. nom. zhémōn Es. Aus den starken casus das ältere lat. hemo, aus den schwachen das altlit. żmu got. guma lat. homo (vgl. Joh. Schmidt, Kuhns z. XXIII, 367 f.). Anzusetzen ist ${ }^{*} \bar{a}^{\prime} \mathrm{cm} \bar{o}$,

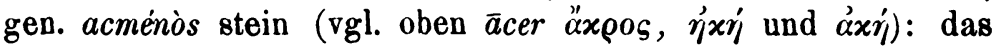
lange $\bar{a}$ scheint im slav. kāmy bewahrt zu sein. cér $A s \bar{o}$ gen. crAsénòs: an. hjassi aus den starken, sanskr. çirșán- (n.) aus

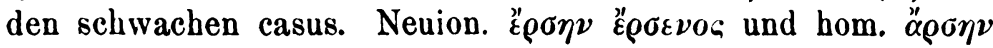
ă ö̌vos weist auf urspr. érsō' rsénòs (Joh. Schmidt, Kuhns. zs. $\mathrm{XXV}, 23)$. Der 'bote' = 'erforscher' hiess urspr. bhéudh $\bar{o}$ ', gen. bhudhénòs: jenes zeigt griech. $\pi \varepsilon v \vartheta i v$, dieses das germa-

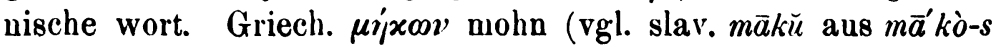

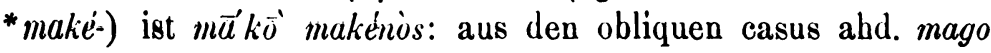
mhd. mage (Wackernagel im wb. setzt kurzen vocal, denselben beweist nach Lexer s. v. das elsäss. mage). Im germanischen hätte es $\mathrm{zu}$ heissen, wenn keine ausgleichung eingetreten wäre (got.) ${ }^{*}$ môha ${ }^{*}$ magins, * fôna fanins fahne (vgl. gr. $\pi \tilde{\eta} v o-\varsigma, \pi \dot{\eta} v \eta$ ), *hôna hanins hahn (vgl. huon huhn, das masc. auf $-n$, das neutr. auf $-s$ gebildet), grôba grabins (grube und graben, in diesem letzten worte ist der ablaut $r \bar{o}: r$ durch $r \bar{o}:$ ro ersetzt), und vielleicht hiess es im germanischen in einer relativ späten zeit noch wirklich so, da das wort ahd. chuocho kuchen, das 
ein lehnwort aus dem roman. ist, im nordischen, englischen und friesischen die form mit kurzem $a$ neben sich hat. Die wörter unsrer klasse sondern sich, wenn wir die gestalt der wurzelsilbe betrachten, in zwei gruppen (s. de Saussure, s. 131 f., 137, 219 f., 229, 231), solche mit $e$ in der stammsilbe, wie $\pi \lambda \varepsilon v \mu o \nu$-, $\tau \dot{\varepsilon} \varrho \mu o \nu-, \sigma \tau \dot{\eta} \mu o \nu-$, und solche mit reducierter stammsilbe wie $\pi v \vartheta \mu \varepsilon^{\prime} \nu, \lambda \iota \mu \varepsilon^{\prime} \nu$-, $\alpha v^{\prime} \chi \dot{\varepsilon} \nu$-, sanskr. uksán- ochse, vidmán- wissen: jene haben die form der starken, diese die der schwachen casus verallgemeinert. ${ }^{1}$ ) Manche haben zur wurzelgestalt der starken den accent der schwachen casus, wie $\chi \varepsilon\llcorner\mu o ́ v ~ s a n s k r$. hēmán-, so besonders viele im sanskrit, andere halten es umgekehrt, wie skr. vr'san-, griech. ${ }^{\prime} \varrho \sigma \varepsilon v$ - — Entsprechend war die flexion der $r$-stämme. Die männlichen nomina agentis auf -tér- -tōr - sind udāttawörter (Sauss. 132): 'träger' hiess also bhértō', gen. bhrtéròs, acc. bhérto' $r m$ (skr. bhártā, aus den schwachen casus altbaktr. beretar-), 'geber' $d \bar{a}^{\prime} t \bar{o}^{\prime}$, gen. datéròs (sanskr. $d \bar{a} t \bar{a}^{\prime}$, aus den schwachen casus lat. dator). ${ }^{2}$ ) Das -tr-der schwachen casus des sanskr. ausser dem locativ -tári, ist eingedrungne tieftonflexion. Von den nomina agentis unterschieden sich ursprünglich die verwantschaftswörter in keiner weise. ${ }^{3}$ ) Es hiess bhra $\bar{a}^{\prime} t \bar{o}^{\prime}$ bruder, gen. *bhratéròs, svésō' schwester, gen. suséròs (in germanischen sind beide stämme erhalten mit dem str aus $s r$ der eingedrungenen svaritaflexion, stark svestr-, schwach sustr-, dies in an. plur. systr aus sustrez und in westund emsfries. suster neben weserfiries. swester), ${ }^{*}$ véscō' ucséròs (lat. uxor). Es muss auch geheissen haben $\left.m \bar{a}^{\prime} t \bar{o}^{\prime} 4\right)$ mutter, gen. *matéròs, * $p \bar{a}^{\prime} t \bar{o}^{\prime}$ vater (nomen agentis von $p \bar{a}$ ernähren),

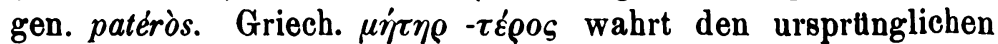
accent des nominativs, sonst haben beide wörter in allen dia-

1) Lat. pulmo ist gewiss nicht lehnwort, sondern gegenüber $\pi \lambda \varepsilon \dot{v} \mu \omega \nu$, skr. klöman vertreter des schwachen stammes, urspr. kuléum $\vec{o}$, gen. kuluménos.

2) Das griech. hat $\delta \omega \tau \dot{\eta} \varrho$, voc. $\delta \tilde{\omega} \tau o \varrho$, aus den starken, $\delta o \tau \dot{\eta} \varrho$ aus den schwachen casus ( $\bar{o}: o$ tritt für $\vec{a}: a$ ein indem das griech. die stufe $d \bar{a}$ aufgiebt und $d \bar{o}$ nach $\delta i \delta \omega \mu \iota$ verallgemeinert. Sonst hat die wurzel Saussures $\varrho$ und das nomen agentis war $d \bar{o}^{\prime} t \bar{o}^{\prime}\left(\bar{o}^{\prime}=\dot{e} \emptyset\right)$ gen. doterós. Das a aber zeigt gr. $\delta \dot{\alpha} v o s$.) Vgl. Joh. Schmidt, Kuhns zs. XXV, 28.

3) Ebenso urteilt Paul, Beitr. VI, $114 \mathrm{u}$. $t \dot{a}-A, a$.

1) Grundiorm, wie es scheint, máAá, dann mit dem pronomen $m \dot{a} A, a$ 
lecten den accent der schwachen casus rerallgemeinert. Ebenso

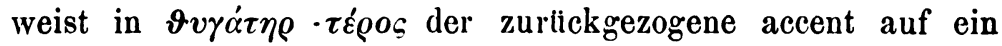
ursprüngliches paroxytonon (*dhéughAt $\bar{o}^{\prime}$, gen. dhug! Atéròs). Homers $\pi \alpha \tau \varepsilon \dot{\rho} \rho \varsigma$ ist in der tat älter als das $\pi \alpha \tau \rho o ́ c$ der eingedrungenen svaritaflexion, wenn diese sich auch im indoiranischen, lateinischen und germanischen wiederfindet: zu griech.

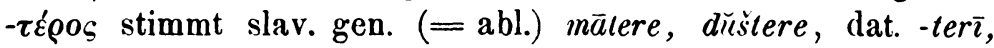
lit. gen. môter's, dukter's. Das ursprüngliche $\bar{o}$ der starken casus habeu im griechischen die adjectivcomposita bewahrt, wie ${ }^{\prime}-, \varphi \iota \lambda o-\mu \dot{\eta} \tau \omega \varrho-\tau o \rho-$ (ganz ebenso wie im germanischen das adj. got. fidur-dôgs die alte starke form des wortes 'tag' festgehalten hat, vogl. das $\bar{o}$ in an. dogr, dän. dogn n.). 'Mann' hiess $\bar{a}^{\prime} n \bar{o}^{\prime}$, gen. anéròs, acc. $\bar{a}^{\prime} n \bar{o}^{\prime} r m$, plur. $\bar{a}^{\prime} n \bar{o}^{\prime} r E s$, griech. gen.

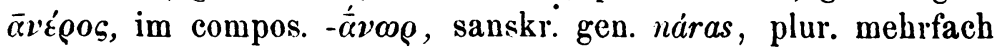
zu lesen $n \bar{a}^{\prime}$ ras (mit der wurzelsilbe der schw. casus, deren anlautendes $A$ lautgesetzlich schwindet). 'Schwager' $d \bar{\alpha}^{\prime} i v \bar{o}$ ', gen. daivéròs: die wurzelsilbe der starken casus ist erbalten in griech. $\delta \bar{\alpha} \varepsilon \varrho^{-}$. 'Schwägerin' éin $A l \bar{o}$ ', gen. inAtéròs: die wurzelsilbe der starken casus in griech. Eivaté in skr. jātár- lat. janitr- (s. Saussure 272). - Die neutra unserer udāttaklasse musten im nom. acc. sing. -on, -or zeigen. Wir finden dies, wie das -ou bei den $u$-stämmen, selten gewahrt: es tritt dafür meistens entweder das $-n,-r$ der svaritaflexion ein (entsprechend dem $-u$ in médhu etc.), oder es dringt das $-\bar{o} n,-\bar{o} r$ des plurals und des masculins ein. $\left.{ }^{1}\right)$ Das $-m n$ der wörter wie $\delta o ́ \gamma \mu \alpha$ teilt sich den udāttawörtern mit, griech.

1) Im germanischen haben wir mehrfach das -on, -or bewahrt, mit dem -cn-, -er- der obliquen casus wechselnd, doch mit iibertritt in die o-declination, also als -an -al -ar (aus -ono-m etc.) wechselnd mit-en -el -er (aus -eno-m etc). Die fälle sind die von Paul, Beitr. VI, 241-246 behandelten, s. u. Unser germanischer infinitiv ist ein ursprüngliches udattaneutrum auf - $\dot{i n}$, dessen dativ im griechischen inf. $(\varepsilon i x-) \dot{\varepsilon} v \alpha \iota$ bewahrt ist. Got. beitan ahd. as. - an ist bheidön, gen. *bhidénos (-an aus -ono-m aus $-\dot{o} n$, sanskr. $-a n a-m$ ist $-e n o-m$ mit dem $-e$ der schwachen casus), ae. bîtan ndhumbr. afries. an. -a dagegen ist bhéid $\vec{o} n$ (-an aus $-\bar{o} n o-m$ aus -on für -on). Ebenso ist unser part. pass. ein udāttawort auf -n (got. ahd. as. -an ae. -en aus -ono- aus -on, got. ahd. as. -in ae. afr. -en mit vorhergehendem umlaut ebenso aus -en-, sanskr - äna- aus -ōno- aus $-\bar{u} n \cdot$ ), bei dem aber im gegensatz zum inf. die stammsilbe der schwachen casus verallgemeinert ist. 
$\sigma \pi \varepsilon \dot{\varepsilon} \mu \alpha, \zeta \varepsilon \tilde{\varepsilon} \gamma \mu \alpha, \chi \varepsilon \tilde{v} \mu \alpha$, sanskr. dhárma, zánma etc. ${ }^{1)}$ Im griechischen sehen wir das -on bei den adjectiven, $\iota d \mu o v$ wissend (mit schwacher stammform), $\dot{\alpha}-\pi \tilde{\eta} \mu o v$, beweisend dass dem neutrum $\pi \tilde{\eta} \mu \alpha$ fruber das $-\mu o v$ zukam, $\stackrel{\alpha}{\alpha}-\pi \varepsilon l \varrho o v$ (aus $\pi \varepsilon \varrho-F o v$ ) ebenso zu $\pi \varepsilon \tau \rho \alpha-\tau-, i v \pi \varepsilon \rho-\varphi \rho o v$ zum verallyemeinerten schwachen stamme $\varphi \varrho \varepsilon \nu$-. Ebenso das -or in neutr. ${ }^{\prime \prime}-\mu \eta \tau o \rho$ etc. Das -or ist im griechischen gewahrt in $\tilde{\eta}$ - $\tau o \rho$, während $\pi \varepsilon l \rho \alpha \rho$ $\left({ }^{*} \pi \varepsilon \rho-f o \rho\right), v \dot{\varepsilon} x-\tau \alpha \rho, \tilde{\eta}^{\eta}-\mu \alpha \rho, \tau \dot{\varepsilon} x-\mu \alpha \rho$, udāttawörter, das $-r$, griech. $-\alpha \rho$ der svaritaflexion angenommen haben. Das letzte wort hat auch das $\vec{o}$ der dreisilbigen formen angenommen, $\tau \dot{x} x-\mu \omega \rho$, ebenso $\pi \dot{z} \lambda \omega \varrho$. Das alte -or ist im lateinischen mehrfach als -ur, im sanskr. als -ar erbalten. Lat. jecur war ljék kuòr $(\bar{e}=\dot{e} E)$, gen. ljEkuénòs: der urspr. nom. acc. ist erhalten in zend jakkare und nit der endung der svaritaform in griech. $\bar{\eta} \pi \alpha \rho$. Lat. femur, gen. feminis, femoris. Im sanskrit haben die hierhergehörigen neutra in den obliquen casus die syncope der svaritaflexion angenommen, gen. skr. jaknás.

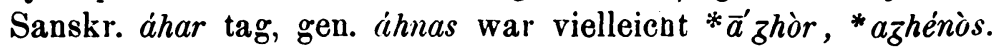
Das wort 'euter' war als udāttawort éuAdhòr (grundf. áuA adhàr,a), gen. üdhénòs (oder -éròs) ( $\bar{u}$ aus $u A$ ): im germanischen haben wir beide formen, die schwache mit $\bar{u}$ in ahd. utar ae udder, die starke mit eu in an. jügr, jüfr fries. iader, iadder. Dies wort hat aber auch als svaritawort bestanden (und ebenso vielleicht noch andere der hier genannten), oùddhr (grundf.

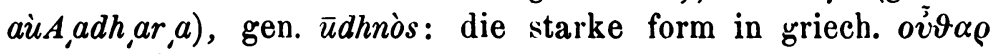
lat. $\bar{u} b e r$ (gen. $\bar{u} b e r i s$ mit udāttaflexion), die schwache in sanskr. $\bar{u}^{\prime} d h n a s$ (nom. $\bar{u}^{\prime}$ dhar mit der endung der udāttaform).

Das indogermanische kann zu der zeit, wo vom udātta und svarita der $a$-vocal gefärbt, svaritierter vocal vor doppeltem anudātta gedehnt und untonige silbe reduciert ward, keine audere flexion gehabt haben als die reinlich geschiedene der beiden grossen gruppen der properispomena und der paroxytona, die wir gesehen haben. Eine jungere zeit erst brachte zahlreiche ubergriffe und ausgleichungen zwischen den beiden gruppen. Eindringen der syncopierenden 'flexion forte' in die obliquen casus der paroxytona haben wir mehrfach beobachtet:

1) Der infinitiv griech. $-\mu \varepsilon v \alpha \iota$ skr. - mánē ist der dativ eines udāttaneutrums auf -mon. 
hei den $i$ - und $u$-stämmen sanskr. dat. sákhiē, griech. gen. vixvos; hei den $r$-stïmmen sanskr. dat. pitrê, mātr $\vec{e}$, svisre $\bar{e}$,

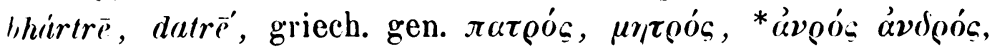
lat. patris etc., umbrisch patres, jünger -er, got. fadrs, brôprs, dann auch weiter in die starken casus, lat. patrem, mātrem, altnord. ace. fotur (aus -rm, Paul, Beitr. VI, 253) etc., plur. fei)r, meotr, systr; bei den $n$-stämmen sanskrit im neutrum regelmässig (nach udnás, aksnás, nắmnas und zahlreichen ähnlichen formen der 'flexion föte') gen. jaknús etc. ${ }^{1}$ ) Lat. gen. vêr-is aus vesr- (urspr. vésòr usénòs frühling).

1) Man wird fragen, wie konnten svaritawörter auf udāttawörter einwirken, wie konnte nécöu-s -évos zu víxvৎ -voৎ werden, da doch jene flexion von der von $x i \rho \vartheta v-\varsigma-v o \varsigma$ ziemlich weit ablag? Was viele neutra die gestalt $-i,-n,-r,-n$ (grich. $-\alpha \rho,-\alpha$ ) statt * oi, -ou, $-o r,-o n$, und viele geschlechtige wörter die gestalt des nom. sing. $-i-s,-u-s$ statt $-(i,-$ ou-s annehmen liess, war die gestalt welche udāttawörter im ersten gliede eines compositums hatten. Im ersten gliede derjenigen schicht der composita, welche den hauptaccent auf dem zweitem gliede trugen, hatten svaritawörter und ndattawörter dieselbe gestalt. Stämme auf $-i$, $-u,-r,-n$ die gestalt $-i,-u,-r,-n$. Also acu- (lat. acu-pedius) von $\vec{o} c u-s$,

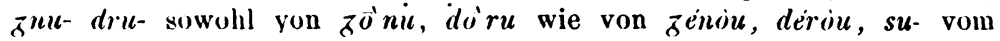

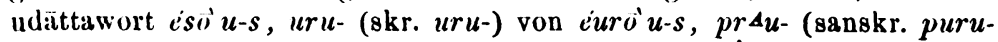
griech. $\pi \circ \lambda v$ - germ. urspr. fulu- was im fries. erhalten), $p, c u$ - (sanskr.

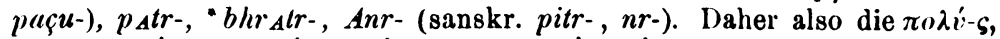
die neutra gr. $-\mu \epsilon$ sanskr. -ma fiir -mon etc. I)as adj. $\mu \dot{\varepsilon} \gamma \alpha-\varsigma$ war als udauttawort auf $-n$ (s. o.) friiher "mezo' neutr. ${ }^{*} m e j z i n$, gen. mzénis, die gestalt des wortes im compos. war also mzn-, griech. $\dot{\alpha} \gamma^{\prime} \alpha$ - (Bezz. Beitr. III, 174, Fick ebd. V, 168$)$ in 'A $\gamma \alpha-\mu \dot{\varepsilon} \mu \nu(\omega \nu$ etc. Jünger trat dafür $\mu<\gamma \alpha-$ ein und danach auch im adj. $\mu \dot{\varepsilon} \gamma \alpha-\varsigma$. (Die starke stufe még- haben wir in an. mikill acc. mikinn diin. megen, die schwache $m g$ - in dem neben diesem herlaufenden an. mykl- (namentlich wie urspriinglich in den obli(fuen casus), aceus. norw. myken schwed. mycken dän. dial. mejen.) Suffixlose svarita- und udättawörter müssen ebenso in diesem compos. dieselbe gestalt haben, also voic-s und veicu-s beide vic- (skr. viç-páti-). Von udattawörtern ist diese gestalt im compos. begreiflicherweise nicht oft erhalten, und die wirkliche zahl der tälle wird für unsern blick noch verringert dadurch, diss wir tälle wie vic- dem svaritawort, nicht dem udāttawort zuschreiben werden. Das udāttawort né 'nicht' erhält im compos. die gestalt $n$-. écvi-s pferd müste in diesem compos. als cuerscheinen: wir finden dies, zu ecu- vervollständigt in as. ehu-skalk. "ycis aus dhesi-s (nach Curtius, wie $\pi \dot{\varepsilon}$ os aus pésos, dagegen soeben J. Wackernagel, Kulins $7 . \mathrm{s}$. XXV, 27(1) wird $9 \varepsilon \varsigma_{-}$in $\vartheta \dot{\varepsilon}(\sigma-\varphi \alpha \tau o \varsigma$ etc. aus dh, s-, oder wahrscheinlich dhes $s$ - mit einem untergeordneten udatta. Für 
Im germanischen finclen wir im masc. der n-decl. grot. Enen. plur. auhsnê, abnê, dat. abnam, altnord. plur. yxn (aus uhsne'z), gen. plur. gumna: diese letzten formen nach der 'flexion forte'

udāttawörter wie menos, sézhos ist zu erwarten ${ }^{*} m n s_{-}^{-},{ }^{*} s, z h s_{-}$, das sich dafür findende ist vielleicht als $s$, zhés-etc. mit unitergeordnetem udatta zu fassen. Die feminine auf $-A$ haben in unserm compos. den alusging

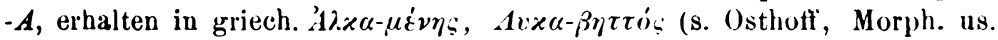
1, 270 anm., wo noch andere beispiele).

Neben dieser schicht von compositen gab es noch eine zweite, die auch der grundsprache zuzuschreiben ist. In dieser behielten beile glieder ihren accent (die spätere sprache gab von den zwei accenten den einen auf), und das erste glied erschien in der gestalt des genitivs, doch ohne das dem gen. angehängte pronomen urspr. -sa $(-s o,-s,-s j o$,

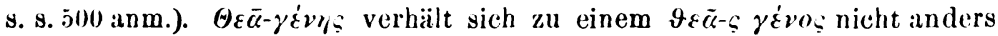
als 'menschensohn' $z u$ 'des menschensohn'. Wir wïden solche composita jenen ersten gegenüber 'uneigentliche' nennen. Wïrter auf $-i-s$, $-\dot{o}-m$ mit dem gen. $-\dot{e}-\boldsymbol{s} \dot{o}$ erschienen also in diesem compositum als $-i-$ mit vorhergehendem anudatta. Zeugnisse sind, wie gewöhnlich, niclrt in der grosse masse sondern in einzelnen als spuren des alten iibrig gebliebenen abnormitäten zu suchen. Zeugnis ist die accentverschiebung bei manchen wörtern auf -o- im ersten gliede von compositen (sanskr. vána-, mëdha- aber vandi-dhiti-, mëdhá-säti- u. lgl.). Sanskr. viçva-aus einem vëicvi-s vicvé-sji erscheint im compos. als vişu, d. i. vicvi-, sivis(s. 501) erscheint im compos. als sve- in got. svi-kunps oftenbar. Die

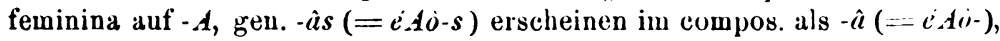
was sich in allen dialecten erhalten hat. Diesem - $a$ bei den udattawörtern auf $-A$ ist nun aber vollständig analog das - $i s i j-,-i v i-,-i r i-$, -eno- der udattawörter auf $-s,-u,-r,-n$, das wir allgemein in Europia und in einzelnen spuren in Asien finden: slav. nebeso- lat. müucri-germ.

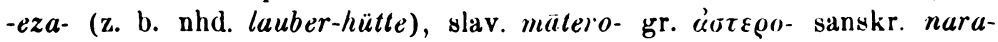
(pati-), slav. kameno- lat. germini-gr. $\varphi$ pvo-. Analog den gesehenen udāttawörtern erschienen die svaritawörter mit deın gen. auf -iss (-il-s,a) in der composition ausgehend auf den svaritierten vocal - $-i-$, also $n k l i j-$,

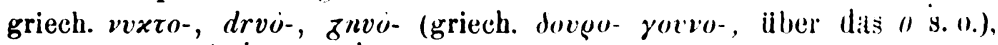
udno- (griecb. ìjoo-, westgerm. vatra-). Bewirkte die erste schicht der 'eigentlichen' composita den übcrtritt von udattawörtern in svaritatlexion, so war es diese zweite, welche zu dem massenhaften eintritt von udataund svaritawörtern in die gestalt und Hexion von o-stämmen den anstoss gab, germ. trevo-, knevo-, rekvezo- etc. aus dem genitiv ohne -s der udättawörter, (mekelo- aus einem ${ }^{*} m e j z j l-s$, gen. mzéli-s, s. o.), westyerm. vatra- und die zahlreichen andern gesehenen fäile ebenso aus dem gen. der svaritawörter. - Einen compositionsvocal -o-, d. h. analogiebildung nach der compositionsgestalt der $o$-stämme fiir alle gesehenen fälle anzunehmen halte ich für unmöglich, denn die vorausgesetzten vorbiliter existierten gar nicht: die o-st:imme erschienen in der composition als 
sind auch nordenglisch, kentisch und friesisch. Die nordfries. mundarten weisen alle auf einen plur. uxen, ixen $=$ an. yxn. Im englischen habeu wir exen, axen (belege s. bei Paul. Beitr. VI, 32). Dem nordischen * gymn, nom. zum gen. gumna, entspricht das me. zemen, zomen (der anlaut zo-, älter ${ }^{*} z^{e o,}{ }^{*}$ z $^{\circ}$ lautlich aus *zix-), ne. yemen, yeomen (schott. yemen men 'common men'), gemeinfreie ('homo mediocris', leg.), zu dem die sprache weil das wort als compos. zu -men erschien, den sing. me. zoman, ne. yeoman bildete. Man kann annehmen, dass diese masculina ursprüglich neben den udāttaformen auch svaritaformen gehalot haben, dass also neben *vécs $\bar{o}$ ucsénòs, zhém

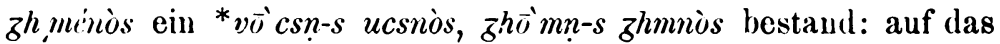
lange $\bar{o}$ eines properispomenons $\bar{\delta} h \bar{o} m n-\dot{s}$ könnte das lange $\bar{u}$ des lat. adj. hümānus zurückgehen, wie $/ \bar{u} r$ aus $b h \bar{o} r$-s entstanden ist. Got. $a b a$ vir wäre als udättawort $\bar{a}^{\prime} p \bar{o}^{\prime}$ apénòs, als svaritawort $\hat{o}^{\prime}$ pn-s apnòs. ${ }^{1}$ ) In einem worte findet sich im

$-\dot{e}-$, und die grosse masse der ersten compositionsglieder von der gestalt

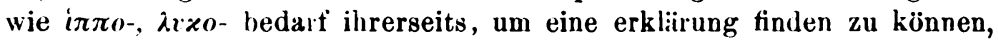

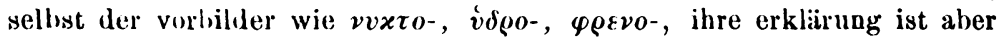

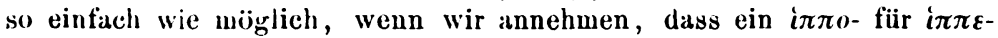
erst intkam nachdem zahlreiche wörter von der gestalt wie vvxro-, o-stämme geworden waren, wie wir dies s. $509 \mathrm{f}$. sahen.

') Den udattavocal haben wir in skr. $\bar{a}^{\prime}$ pas $\mathrm{n}$. neben apas aus $a^{\prime}$ pos apésos, und vielleicht in ahd. uoba, der svaritavocal $\bar{o}$ hat bestanden in " $\ddot{\prime} p$-s *apos f., aus dem das lat. op- (gen. opis etc., in-op-s, $(0 p$-s) hervorgegangen ist. Das lat. opus $\mathrm{n}$. = sanskr. apas hat sein $o$ (wenn die wurzel nicht statt $\operatorname{des} A$ Saussures $g$ hat) von diesem opempfangen oder von einem svaritawort auf $-s,{ }^{*} \vec{o} p s{ }^{*}$ apsis, dann - opsos, das im ostitalischen (in der sprache der von dieser wurzel ihren namen tragenden '(Juxoí) als ops- (üps-) im verbalstamme opsá- vorliegt. Das von Kluge, Kuhns zs. XXV, $31 \%$ zusammengestellte ved. apra- $=$ got. abr-s stark war ursprünglich entweder ein udättawort $a^{\prime} p r \dot{0}-s$ apré-, oder ein svaritawort o' pr-s apró-s.

Las wort 'atem' war als udattawort étmo-s Atme- (jenes in as. $\hat{a} \partial o m$ ae. $\hat{a} \partial m$, dieses in griech. $\dot{a} \tau \mu \dot{\theta}-\varsigma$, ahd. $\hat{a} t u m$ setzt den accent der schwachen casus voraus) und $(+n a)$ étmò Atménos (altfries. èthma, sanskr. ätmá, aus den obliquen casus sauskr. tmain-), bestand aber daneben als svaritawort $\vec{o}$ 'tm-s Atmos (uhd. òdem, griech. $\alpha \tau \mu o ́ s$ kann auch aus den obliquen casus dieses wortes hervorgegangen sein) und $\vec{o} t m n$ * Almuos (altfries. omma). Das $A$ dieses wortes, das mit vorhergehendem $\dot{e}$ langes $e$ giebt, muss ein anderer laut gewesen sein, als das $\boldsymbol{A}$, das sich mit vorhergehendew $e$ zu langem $\bar{a}$ vereinigt, s. o. s. 493 anm. Dassselbe 
fricsischen sicher lubertritt aus der 'flexion faible' in die 'flexion forte', im dual des udāttawortes céivō civéjòs (s. 515), dann fürs germ. * civénòs, 'chegatte' [vgl. skr. céva- und civá 'hold' aus cíivò-s civé-, s. jetzt Noreen oben s. 441 f.], der, mit dem $-\bar{\emptyset}$ des duals masc. der 0 -decl., germ. heivōnō zu lauten batte (vol. as. sin-hinun ae. sin-hivan): das altfries. Rlistr. sin-higen ist aber ein heivni,, = an. hjön du. ehegatten, dazu gen. dat. emsfries. hiûnu. hîunum, Rüstr. hiônon (das hiûn-, hiôn- aus germ. hiun-).

Umgekehrt greift auch die 'flexion faible' tuber ins gebiet der 'flexion forte'. Bei den $i$ - und $u$-stämmen im sanskrit regelmässig lat. gátajē, kêtáve (d. i. -téjai, -tévai statt -tiái,

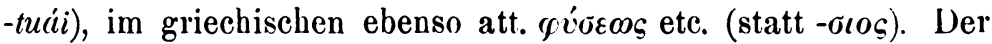
$r$-stamm 'wasser', urspr. svaritawort, nimmt im griechischen im nom. acc. sing. die endung eines udāttawortes, wie $\tau \dot{\varepsilon} x \mu \omega \varrho$, an, $\ddot{v} \delta \omega \varrho$, und $\mathscr{v} \delta \omega \varrho$ mit der vocallänge des alten svaritawortes (wie es scheint also mit ersetzung des verbältnisses $f \omega: v$ durch $\bar{v}: v$ ). Im sanskrit tritt bei den svarita- $n$-stämmev, wie $n \bar{a}^{\prime} m a$, der nom. acc. plur. regelmässig in die udāttaflexion ein, $n \bar{a}$ 'māni. Im lateinischen herscht völlige 'flexion faible', nōminis (aber umbrisch abl. nōmne), ebenso im slavischen, gen. (abl.) imene, dat. imeñ̄. Im gotischen herscht in den

element kann in got. jèr (jéroj-m) abaktr. järem n. jahr gegeniiber dem

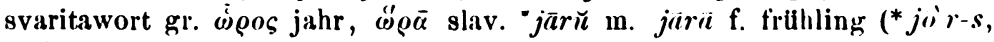
* $j(r A$ ) bestanden haben.

Svaritaformen neben den udättaformen liegen den verschiedenen gestalten eines von der wurzel zava $(z u)$ kauen durch -sa und sa-na abgeleiteten wortes für 'backenzahn' in den germanischen dialecten zum

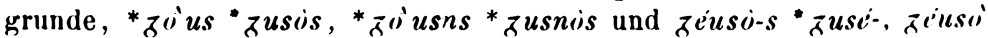
-zusénos: das svaritawort auf $-n$ hat im norlfriesischen im plural dic 'Hlexion forte' bewahrt, Amrum kìj' (aus kîja m.), plur. kìj jin.

Uebertritt von der 'flexion faible' zur 'flexion forte' sehen wir in den beiden wörtern 'hund', urspr. ceivo' (air. cü) cue'nos, acc. cevi' n-m, und dem $m$-stamm zhéjöm-s, gen. zhiemo-s (+na: zheimö zhiminis). (ìriech.

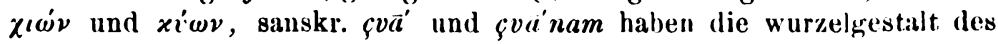
schwachen stammes verallgemeinert. Sanskr. ̧̧üas griech. xivic und abaktr. gen. zimo (aus zhimos) sind eingedrungene svaritaflexion. Das $\dot{e}$ der udättaflexion ist im lateinischen erhalten in gen. hiemis und in canis (das ein $e$ verlangt, lat. va aus ve wie in quattuor. Joh. Schmidt erkliirt KZ. XXV 49, das $a$ in quattuor durch den iibergang von ktvrin cvar-, aber nicht $r=$ skr. $r$ gr. $\alpha \rho$ lit. $i r^{\prime}$ sondern $r^{A}=\mathrm{skr}$. $i r$, ïr gr. oo lit. ir wird lat. ai, wie in den von Joh. Schmidt angefihrten tiallen.). 
obliquen casus des singr. 'flexion faible', gen. namins, vatims: im plural haben namna, valna die 'flexion forte' bewahrt. Ebenso zeigt das oben angesetzte $p \bar{o}$ 'uni * punnòs dieselbe 'flexion faible' im sing., fôn funins.') Die wörter 'herz', 'ohr' und 'auge' sind ursprïnglich *cordi (aus car'daj,a) oder (wenn cìradaja zu grunde liegt) ${ }^{*} c \bar{c}^{\prime} r d i$ (sanskr. $\left.h \bar{a}^{\prime} r d i\right)^{2}$ ), gen. crdnòs, $\bar{o}^{\prime} u s i(\bar{o}=o A)$, gen. ausnòs, ${ }^{*} \bar{o} k u i(\bar{o}=o A)$, gen. ak'nòs. ${ }^{3}$ ) Im gotischen haben 'auge' und 'ohr' 'flexion faible' angenommen, nicht allein in den obliquen casus des singulars, augins ausins, sondern auch im plural, -ôna. Im schwedischen und dänischen haben diese wörter im dual oder plural (was hier dasselbe, germ. augnō', auzuō' mit der dualendung des masc. der $o$-(lecl.) die 'flexion forte' gewahrt, altschwed. oghun, srun, altdän. oghoen, orcen, im friesischen in allen dialecten nur das wort 'auge', altfries. Riistr. âgon, Ems. âgen, während ira 'flexion faible' angenommen hat. (Ueber den ahd. an. plur. auf $-u n,-u$ s. u.) Ile wurzelsilbe des germanischen wortes 'herz' mit dem vocal $e$ kann weder aus der svaritierten silbe der starken noch aus der reducierten der schwachen casus des svaritaworts hervorgegangen sein, es nuss also neben dem svaritawort gen. cṛdròs, ein udattawort, cierdòn, gen. crulénòs, bestanden haben: dieses letztere ist unser germanisches wort, und dieses wird mitgewirkt haben den ubrigen gesehenen formen, augins, funins, vatins, namins, die 'flexion faible' zu erteilen.

Nicht genug an der ausgleichung zwischen den syncopierten cndungen der 'flexion forte' (-i-, - -u-, -r-, $-n-)$ und den den vocal $e$ enthaltenden der 'flexion faible' (-ci-, -ent-, -er-, -en-), auch die in der 'flexion faible' neben einander bestehenden

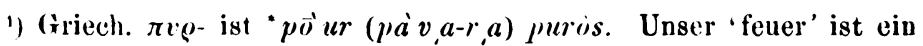
udattawort (urspr. "piuroi n., gen. purcijos"?, dann peuri).

$\left.{ }^{2}\right)$ Ebenso weist sanskr. ma'rz-mi auf $m \ddot{o} r_{\bar{\kappa}-m i}$ (aus dreisilbigem miraza).

3) und òksi, geu. aksnost(sunskr. aksi, aksnas). - Alle drei wörter bestehen aluch als consonantische stämme, cor $d$ (oder $\left.c i^{\prime} r l\right)$ gen. crd $j-s$

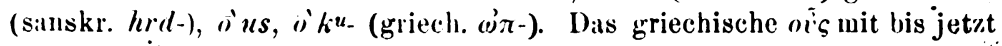
merklärlichem " verdankt dieses einfach dem iibergang des ablauts (1) : $\alpha$ in $(1): 0$. das hom. o'at- dor. (jat- att. (j) $\tau$ - der obliquen casus ist ousn-l-. Dasselbe ist das $o$ in ö $\sigma \sigma \varepsilon$, oculus gegenïber armen. akn (s. s. 493 anm.). 
$e$ und $\bar{o}$ der endungen im mase. und fem.. $e$ und $o, o$ im neutrum verfielen dem ausgleich. Es treten folgende fälle ein:

1. Der dunkle (ursprünglich sraritierte) vocal sient.

a) $o$ verdrängt das $e$, der ablaut $e: \bar{o}$ geht iiber in $o: \bar{o}$.

b) $o$ verdrängt das $\bar{o}$.

c) $\vec{o}$ verdrängt das 0 .

2. Das $e$ (der ursprüngliche udāttavocal) siegt.

a) Das $e$ verdrängt das $\bar{o}$.

b) Nach dem vorbild des verhältnisses $o: \bar{\sigma}$ wirl ein $e: \bar{e}$ geschaffien.

c) $\bar{e}$ verdrängt das $e$.

1. a) Ein zum $o$-vocal der starken casus passendes $o$ ver-

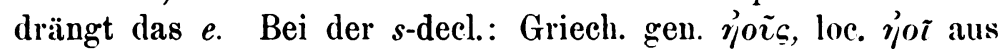

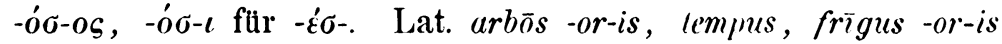

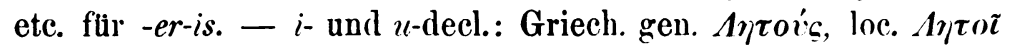

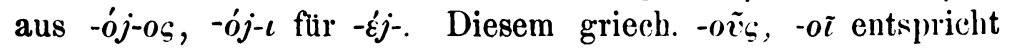
aufs genaueste das got. -ais, -ai. mit dem ihm analogen -aus, $-a u$. Jenes ist in der gestalt vorgerm. $-o j-o s,-o j-i$ entstanden in den udāttawörtern mit dem nom. auf $-\bar{o}$, und hat sich von diesen den sämtlichen weiblichen $i$-stämmen mitgeteilt, wie der nom. sing. auf $-\bar{o}$ im nordischen. Das -aus, -au (alteugl. altfries. - $a$ etc.) gehört zu den neutren, für welche oben die nominativendung -ou nachgewiesen ist, wie frécò, grerm. fíhou, und zu den ihnen firiher \%ur seite stehenden greschlechtigen wörtern auf -ōu-s (wie sunu-s, s. s. 514 anm.): zu pécou trat gen. pecovos fur -iv-os, genau wie zu tempus lemp-or-is. -

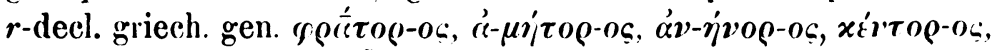

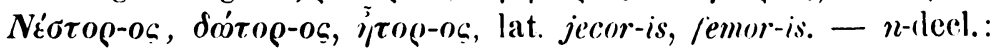
griech. gen. $\pi \lambda \varepsilon \dot{v} \mu o \nu-o \varsigma$ etc., $\alpha-\pi \eta_{\mu}^{\prime} \mu \nu-0 \leq, \not \alpha-\varphi \rho o v-o s$. Auf germanischem boden finden wir dieses -on im gren. dat. sing. als -an- im altsächsischen, gen. dat. sing. as. herran (belege s. bei Paul, Beitr. IV, 360) zum nom. herro, ace. herron. Im gotischen finden wir dies -an-aus -on- im gen. plur., han-an- $\hat{e}$, hairt-an- $\hat{e}$ und es ist auch im dat. plur. vorauszusetzen. Dieselbe form mit -on- liegt dem nordischen gen. plur. floma, gotna (gewöhnlich hana nach der o-decl.), hiartna zum grunde. Genau dasselbe wie das hier gesehene ist es, wenn zum nom. -o-s acc. -o-m, plur. oos der o-stänme der gent. -o-so (s. 5110 
anın.), -o-s.jo für r-so, -ei-s.jn, der locativ -oi fïi! -ei, u. s. w. sich einstellt.

b) Das o verdrïngt das $\bar{o}$ in den ursprünglich dreisilbigen starken casus. In griechischen allgemein, wo es in die obliquen

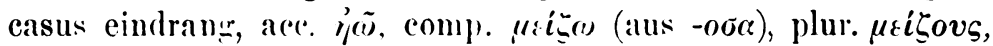

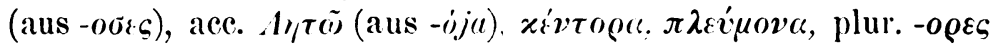

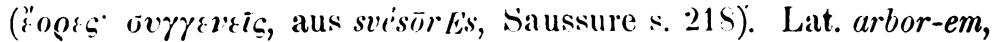
- $\bar{e} s$, plur. neutr. tempora, jemora (neben dem älteren meliōra). Altengl. plur. neutr. lambru, cildru, dem lat. -or (a) entsprechend mit -os- fiìr -ñs-. Altnorl. -ir (sóltir), plur. mase. und fem. der i-decl., zunïchst aus -aiz, dies aus -oj-es. Altengl. -a (suna), ahd. $-\infty$ in dorno, plur. der u-decl., ebenso aus -auz aus -ov-es (dagegen altengl. $-u$, sumu, im gen. loc. sing., nom. plur., entsprechend dem aurh im gotischen rorkommenden gen. daupus, loc. vulbu, ist das -uos, -ui. -ues der 'flexion forte'). 1) Im altsächs. dringt das -an =-onos, -oni auch in den ace. sing., frôhan, millean, aber nicht in den plur. (s. Paul, a. a. o.). Im rotischen (nicht im nordischen) ist das -an aus dem gen. plur. $-a n-\dot{e}$ auch in den nom. (ace.) plur. hanans und von diesem auch in den are. sing. hanan gedrungen, aber nur im masc., nicht im neutr.: got. -an, -ans ist $=$ griech. -ova; -ov\&s, welchem gegeniiber huggònıs, hairtôna das ältere -ōn-festhalten. Dem srot. -an in hanan scheint analog zu sein das -ar in brôpar, fadur (das dann auch in den nom. gedrungen ist) aus -or-om (mit ler endung $-o m=$ sanskr. am der $o$-decl. $)=$ griech. -oge in sgúteogre: das -or- wïre dann auf demselben wege an diese stelle gelangt, aus einem friberen gen. plur. * fadarê.

c) Das $\bar{c}$ der dreisilligen starken casus dringt auch in die zweisilbigen und in die schwachen. Nirgends ist dieses eindringen des "aus dem ace. singr., nom. ace. plur. in die schwachen casus beliehter als im lateinischen: bei den $s$-stämmen homör-is, $r$-stämmen sorör-is, uxīr-is, emptōr-is, n-stämmen

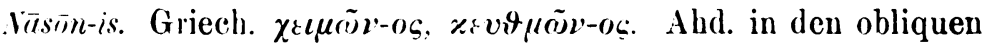
casus des plurals der $n$-stïmme aller geschlechter, hanôno,

1) Gotisches im ace. sing. vorkommendes -au, hairau, wozu dann anch in nom. sing. -aus, sunaus, fairhvaus (die fälle s. bei Leo Meyer, Got. spr. s. 5it), künnte wöglicherweise urspriinglich den an zahl geringeren wörtern der 'flexion taible' zugekummen sein, -au aus -ov-om tiir $-o v-m$. 
zungôno, herzôno, genau = lat. -̄̄num ırr. óvov. Im engrlischfriesischen entspricht die $n$-declination des masc. (s. u.) in

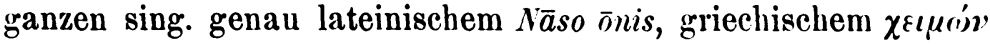
$\tilde{\text { wyos }}$, das constante ae. -an fries. - $a$ kann im masc. nur aus - $\bar{n} n$ - entstanden sein. Der ae. plural -an aus -ōnes kann nicht dem got. -ans entsprechen, das ae. -en wäre. - Eindringen des $\bar{o}$ aus den dreisilbigen in die zweisilbigen starken casus sehen wir im gotischen und nordischen im nom. acc. sing. der $n$-stämme: die endung war bei den paroxytonen, wie oben gezcigt, ursprunglich -on, cérdòn, dann nach dem plural on, cérdōn, germ. hértōn, got. hairtô an. hiarta.

2. a) Das $e$ der schwachen casus verdrängt das $\bar{n}$ der dreisilbigen starken. Bei den $s$-stämmen: plur. neutr. statt $-\bar{o} s-a$

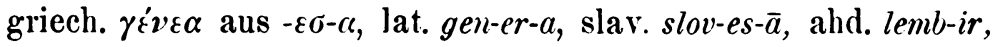
huon-ir, altnord. hen-s. Bei den adjectiven acc. sing., nom. plur. -es-m, -es-es statt -ōs-m, -ōs-Es sanskr. su-min-as-am, su-

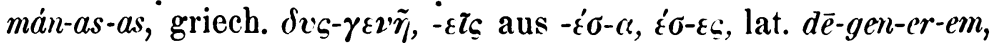
$-\bar{e} s$. - Bei den $n$-stämmen acc. sing. -en-m, nom. plur. -en-es statt $-\bar{o} n-m,-\bar{o} n-E s$ : sanskr. uksinam, vr'sanam, -anas (neben

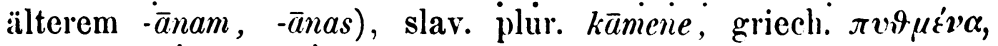

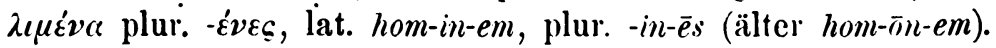
Im plural des neutrums - en-a fiur -ōn-a, das dann auch in die flexion der urspr. properispomena eindrang: slav. imeen-a, lat. $n \bar{o} m-i n-a$. - Bei $r$-stämmen acc. sing. -er-m, nom. plur. -èr-es fuir -ōr-m, -or-Es: sanskr. pitáram, mālúram, pituiras, matúras

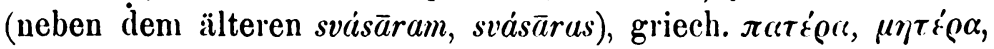

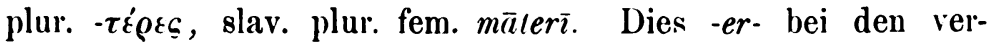
wantschaftswörtern, und nicht einmal bei allen, im gegensatz zum -ōr- der nomina agentis, ist um nichts altertumlicher als das -en- neben dem -õn-bei den n-stämmen. Neben dem gr.

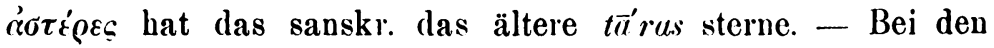
$i$ - und $u$-stämmen findet sich im acc. sing. das $-e j-m$, $-e v-m$ für $-\bar{o} j-m,-\bar{o} v-m$ (oder $-\bar{o} i-m,-\bar{o} u-m)$ nur sehr sporadisch, weil hier das $-i-m,-u-m$ der properispomena den vorsprung gewonnen

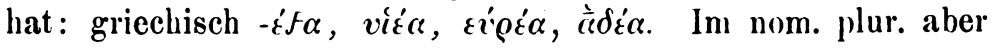

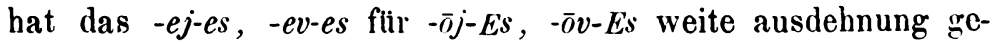

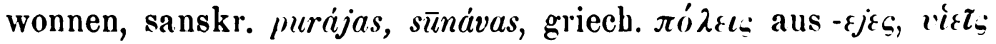
aus $-\varepsilon f \xi \xi$, germanisch -ejez in got. -eis ahd. $-i$ ae. $-e$, -evez in got. sunjus an. synir, slav. sȳnove (ov lautlich aus $e v$ ). Auch 
dieses -ejes, -eves, obwohl es auf allen gebieten des indogermanischen sich findet, ist so wenig ursprtinglich wie das -enes, und ist nicht der grundsprache zuzuschreiben. Das got. -eis etc. aus -ejes des gotischen und westocermanischen ist um nichts altertimlicher als das nordische $-i r(=a i z)$ aus -ojes: dieses hat im gegenteil die dunkle vocalfärbung als grössere altertimlichkeit voraus. Im plur. neutr. haben wir -ev-a für

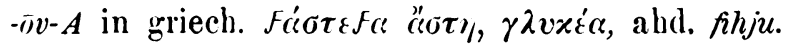

b) Die wörter, in denen das o das $e$ verdrängte, sind natürlich, nicht durchaus, aber vorwiegend, zugleich solche, in denen der accent der casus mit dem o-laut, also der starken, den der casus mit dem $e$-laut oder der schwachen tiberwältigte, die wörter sind also grösstenteils im nom. sing. paroxytona (und danach in den dreisilbigen casus proparoxytona). Die wörter, in denen das $i$ der schwachen casus das o der starken verdrängte, haben dagegen natirlich auch in der regel den accent der schwachen casus verallgemeinert, es sind also vorwiegend im nom. sing. oxytona, in den dreisilbigen casus paroxytona. Nach dem vorbilde nun des in jenen wörtern mit dem $o$ der schwachen casus wechselnden $\bar{o}$ des nom. sing. wird in diesen wörtern zum $\dot{e}$ der schwachen casus ein $\bar{e}^{\prime}$ für den nom. sing. geschaffen. Bei den $A$-stämmen stellt sich zu dem den udāttavocal $;$ enthaltenden $-\hat{a} s$ des gen. sing. ein $-\vec{a}^{\prime}$ austatt des alten $-\bar{o}$ im nom. sing ein, griech. $\tau \iota \mu \eta \dot{~ z u ~} \tau \iota \mu i j \varsigma:$ es wird nämlich anzunebmen sein, lass dies $\vec{a}$ sich zunächst bei den wörtern einfand, die den accent der schwachen casus verallgemeinerten. Bei den $s$-stämmen tritt im adjectiv zum - $i s-$ der obliquen casus ein $-\bar{e}^{\prime} s$ für ailteres -ì's im nom. sing. II. f., cin -is fur -is im neutrum: sanskr. jaç $\bar{a}^{\prime} s$, lava $\vec{a}^{\prime} s$, wahrscheinlich mit i weyen des ace. risam, neutr. jaçis, taveis,

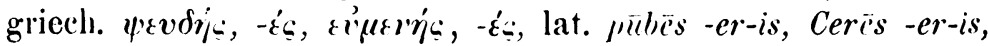
subst. $s \bar{e} d \bar{c} s$ etc., in die $i$-decl. übergetreten. Dass die adjective mit diesem $\bar{e}$ und mit diesem aecent nicht aus der grundsprache stammen können ist klar: die vorhergehende silbe hat die gestalt, wie sie nur die silbe haben kann, die in der grundsprache den udatta trug, dieselbe welche die zugehörigen substantive unter dem accent zeigen. Ursprtinglich kann die accentuierung keine andere gewesen sein als $s u-m i-n i \bar{s}$, neutr. su-ménòs (das sanskr. wahrt diesen accent), gev. su-mnésòs, acc. 
mase. su-ménò'sm. Die arljective haben mọ̈lichst „renau die gestalt der ihnen zur seite stehenden substantive gewahrt, die indogerm. dialecte sind aber, unabhängig oder noch in fühlung miteinander, darauf verfallen im adjectiv den accent der obliquen casus, im substantiv den der starken zu verallgemeinern, um so adjectiv und substantiv zu differenzieren. i $\gamma \bullet \dot{\eta} \varsigma$, ein adj. ohne zur seite stehendes subst., zeigt die urspringliche wurzelsilbe der obliquen casus, urspr. v' $\dot{c}^{\prime} \bar{o} s, v \dot{c} z \grave{\delta} s$, gen. užésòs (ugjés-wäre

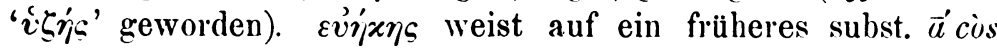
acésòs. Vyl. Kz. XXIV, 33, 109. - Bei den $r$ - und $n$-stïmmen haben im griechischen ungekehrt die adjective, wie wir gesehen haben, den o-laut gewahrt, wo die substantive das $\dot{e}$ ver-

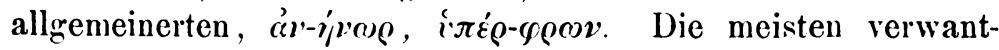
schaftswörter auf $-r$, aber nicht alle, nehmen zum -ér- der obliquen casus im nom. sing. $-\bar{e}^{\prime},-\bar{e}^{\prime} r$ an statt des alten $\bar{o}^{\prime}$ und (mit dem aus den iibrigen casus eingedrungenen $r$ ) $-\bar{o} r$. Sanskr.

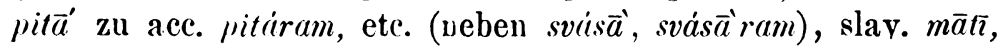

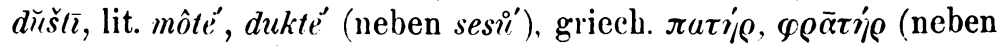

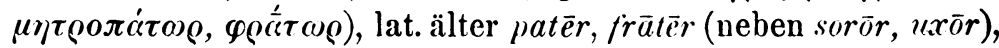
altir. athir (neben siur), altnord. allgemein faðir, bróðir etc. ${ }^{1}$ ) Im griechischen haben zum teil auch nomina agentis das - $\tau \dot{\eta} \varrho$

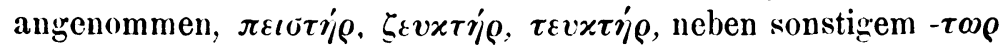
lat. $-t \bar{o} r$. Ganz ebenso haben wir bei den $n$-stämmen im nom. sing. $-\bar{e}^{\prime},-\bar{e}^{\prime} n$ statt des $-\bar{o}^{\prime}$ und dazu getretenen $-\bar{o}^{\prime} n$. Sanskr.

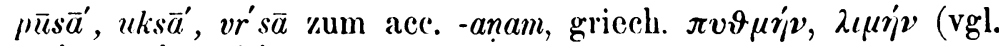
Paul, Beitr. IV, 353$)$, lat. pectēe, liēn (lann -ền). Man ist durchaus nicht dazu berechtirgt, alle diese vorgänge, das eindringen des $i$ in die starken casus und die entstehung des $\bar{e}^{\prime}$ im nom. singr., bei den $r$ - und den s-stïmmen der grundsprache zuzuschreiben, wenn man sic bei den $n$-stïmmen in dic einzclsprachen verlegt.

c) Das $\bar{e}$ dringt in die schwachen casus an die stelle des

1) Gemeingermanisch weiss ich diesen nominativen das e nur diann zu erteilen, wenn das $-r$ erst in junger zeit nach der kiirzung ausliutender lïngen in den einzeldialecten hinzugetreten ist: germ. * fade' muste wriden an. "faji got. * fada ahd. *fate ae. "fade und wach dem gen. * fade. Sonst ist got. fadar der acc. -or-om, ahd. fater der acc.-er-om. Das altengl. brijor (sweostor, dohtor, mòdor), plur. brojjor ist am wahrscheinlichsten altes bhri'tor acc. -or-om, plur. bhrätöres. 


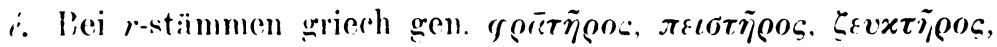

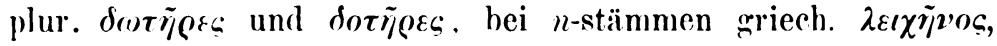
z\&vijujos ete., lat. liēnis. Bei udätta $-i$ - und -u-stämmen (s.

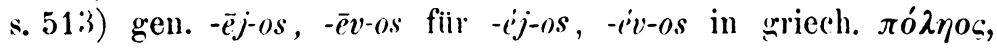

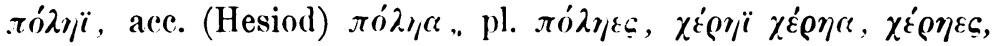

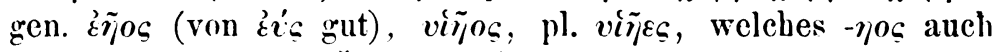

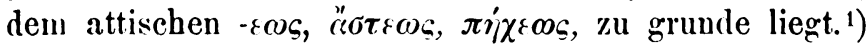

Die entstehung aller dieser von uns zuletzt betrachteten umgestaltungen der ursprünglichen flexion werden wir nicht in eine $z u$ friihe zeit verlegen dürfen. Im griechischen dialecte mōgen sie etwa in jahrtausend vor Homer eingetreten sein, soweit sie nicht noch jünger sind. In den nordeuropäischen dialecten sind sie gewiss jünger. ${ }^{2}$ )

1) Wie in den gesehenen fällen in der nebensilbe so ist auch in ainigren fällen in der stammsilbe fiir das urspriingliche svaritierte $\vec{\sigma}$ ein i eingetreten. Wir haben lat. lég- f. neben germ. laga- n. geset\%. Als grundform fiir beides ist anzusetzen $l \vec{o}^{\prime} g h-s$, gen. lghos. In den obliquen casus muste lat. ${ }^{*}$ leg- entstehen (vgl. levis = Ėaciç, lat. ncc-, wovon nom. nex), zu diesem $e$ der obliquen casus hat sich in den starken anstatt des $\bar{o}$ ein $\ddot{i}$ eingestellt, das dann das $e$ der obliquen casus verdrängte. Vom starken stamme lōgh- abgeleitet haben wir im germanischen das verb an. logga ae. lôzian altfries. ùt-lôgia gegeniiber dem gleichbedeutenden lat. lëgare (s. Kuhns as. XXIV, 442). Im germanischen hat sich dagegen umgekehrt zum lögh- der starken fiir die obliquen casus ein logh/- eingestellt, das dann in die o-declination iberging.

lin schwierigeres wort ist germ. kvēui-z, got. kvêns, = skr.-zāni-, beides aus $g^{u \bar{e}} \| i-s$, das aber ein svaritawort, $g^{u} \bar{o}^{n} n-s$, gen. gunios, gewesen sein muss. Nach dem vorbild der wörter mit $i: \ell$ wird sich in den obliyuen casus ein guinios (sanskr. zimi-) eingestellt haben, und dies $c$ hat damn in den starken casus ein $e$ fiir o hervorgerufen. Fïr etwas urspriingliches kann das : fïr erwartetes 0 in solchen wörtern nicht angesehen werden.

2) In dem voraufgehenden excurs iiber die entstehung des $o$ werden zahlreiche differenzen zwischen den indogerm. dialecten als solche beseitigt, und durch einen urspriinglichen wechsel des accents in der flexion erklärt. Ich hoffe, dass durch diesen wechsel noch manche von mir nicht angefiihrte differenzen ihre erklälung finden werden, die mir jetzt entyangen sind, weil es mir augenblicklich an biichern fehlt. Ich habe diese arbeit leider an einem orte und zu einer zeit machen muissen, wo mir nur ïusserst wenig biicher zu gebote stchen: nicht einmal alle in diesem aufsatz citierten habc ich bei der abfassung wirklich zur hand gehabt. Am nuitzlichsten ist mir saussures buch gewesen, da F. de 
Saussure alles seinen ansetzungen widersprechende aufs gewissenhafteste verzeichnet.

Das verbum, welches das nomen zur voraussetzung hat, muss in accentwechsel und ablaut genau zum nomen gestimmt haben. Svaritaflexion: roikmi (lit. lëku), rikmési. Für unsere germanischen svaritaverben (mit redupl. im perfect) brauchen wir im prïsens keine reduplication, ahd. skeidu aus skoitmi skitmési, stòzu aus stoùdmi studmési. Der übergang aus der consonantischen in die $o$-flexion ist genau derselbe, wie der oben 8 . $509 \mathrm{f}$. beim nomen verlangte. Ahd. tuom ist $d h \vec{o} \mathrm{mi}$ *dhEmési. Bei festhaltung der abstufung fielen die verben mit den präteritopräs. zusammen, got. daug, dugum ist dhoughmi gebe milch, ertrag (sanskr. dohmi melke, duhmas). - Die udattaflexion muss ursprïnglich gewesen sein rikit, rikisi, rikiti, prät. i riikiom, i rikis, $i$ riket (secundärsuffix $-m,-t,-n t$ aus $-m a,-t a,-n t a$, primär $-m i,-t i$, $-n t i$ aus -ma-ja etc., medium -tai, -ntai aus $-t a-A, a-j a$ etc.). Vgl. Fick, Bezz. beitr. IV, $17 \tau \mathrm{ff}$. $v \bar{a}^{\prime} d \vec{o}^{\prime}$ vade'si: aus jenem lat. $v a \bar{a} d o$, aus diesem ae. wadan. Ebenso verhalten sich ae. ceorfe und griech. $\gamma \rho \alpha \dot{\varphi} \omega$, griech. $\tau \rho \dot{\pi} \pi \omega$ und $\tau \rho \dot{\alpha} \pi \omega$. westgerm. tredan und ostgerm. trudan (urgerm. trejdö trudisi). Prät. é dircòm, id drce's. Wie nun $\sigma \tau \dot{\alpha} \mu \omega \nu{ }^{*} \sigma \tau \alpha \mu \dot{\varepsilon} \nu o s,{ }^{*} \lambda \varepsilon \dot{\mu} \mu \omega \nu$

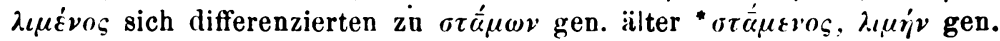
$\lambda \iota \mu \dot{v} \nu \circ \subseteq$, genau so gingen in nachgrundsprachlicher zeit auseinander präs. rëiko rëikesi, prät. ¿ rëikom, $\dot{e}$ rëikes und präs. riké rikési wozu prät.

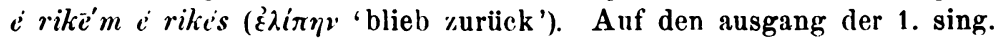
präs. - $\bar{e}^{\prime}$ im falle der oxytonierung, vad $\bar{e}^{\prime}$ neben $v \bar{a}^{\prime} d \bar{o}$, weist die endung der 1. sing. $-e$ im englisch-friesischen zurück: das englisch-friesische setzt nämlich durchaus die betonten endungen -isi, -eti, - $\dot{\text { nnti durch }}$ sein $-s,-p$ voraus. Nur die östlichen dialecte legten in die doppelheit $i$ rikkom und $i$ rikom einen unterschied der bedeutung hinein, das griechische den des dauernden und eintretenden. $\dot{e}$ riké' $m$ verallgemeinerte später sein $e^{\prime}$ (s. oben $2 \mathrm{c}$ ), $i^{\prime}$ riké's etc. Das griechische brauchte dann $i$ rikom transitiv, e rike' $m$ intransitiv. Das germanische scheint nur gekannt zu haben prïis. réikō - e'si oder vadié e'si, prït. rikè'm -ës, vadém - ís (altnord. kera, frera, -ir, -i aus zusém, prusé'm, -ё's, -ét, vgl. lingl. stud. III, 161, ahd. forahta aus prkt-é'm, -i't zum prïisens prktō. Nebeu diesem starken prät. -èm besass das germanische ein schwiches prät. auf -täm, wie das latein. sein -bam: von jenem starken prät. auf $-\bar{c}$ 'm hat das gotisch-nordische die constanten endungen des prät. $-\bar{e} m$, - $e s$ etc., während das hochdeutsche das -äm -ăs des schwachen prït. verallgemeinert hat, ahd. 2. sing. forahtòs aus $-\bar{a}^{\prime} s$.) - Den accentwechsel des wurzelverbs auf -o muss auch das präsens auf -jo, -no, -to gehabt haben (s. das nomen, s. 501), also virz-jó ( $\left.\dot{\rho}^{\prime} \zeta \omega\right) \operatorname{vr} z-j i-t i$ d. h. 'ich wirke, er wïrkt', sä́gjö (sôkja) sagjeiti (sagio). Auf einen ehemaligen accentwechsel bei den schwachen verben deuten ziemlich zahlreiche fiille wie födjan gr. $\pi \alpha \tau \dot{\varepsilon} \circ \mu \alpha \iota$, auf einen ehemaligen regelmässigen wechscl zwischen dem $o$ und $c$ derselben verben fälle wie die s. 473 angeführten got. pahan list. taceo etc. (s. den nachtrag). 
Die personalendungen und ebenso die casussuffixe miissen urspriinglich je nach der accentlage verschiedene gestalten gehabt haben. So ist das oben angesetzte rikés, rikési, rikmési, wie ein locativ auf -éi, -ivi, -eri, -eni, nach dem accentgesetz unmigglich, doch muss ich es der weiteren forschung iiberlassen für diese suffixe die ursprünglichen gestalten zu finden. Angenommen das primärsuffix der 1. plur. akt. war urspr. -ma-sa-ja, das secundärsuffix -ma, dann muste es in der svaritaflexion heissen 1. plur. *rik-mésoi, secundär rik-mé, in der udāttaflexion 1.plur. réikömsi, secundär * rëikom nach dem gesetz über dio dehnung des svaritierten vocals vor doppeltem anudātta (s. 49s). Das indoiran. lange $\bar{a}$ der 1. dual u. plur., sanskr. -āvas, -ämas, secundär -āva, -äma wäre also von den primären endungen her verallgemeinert, das europäische $o$ in griech. $-o \mu \varepsilon v$ got. $-a m$ von den secundären. Das gotische - $\hat{s}$ der 1. dual. muss ursprïngliches langes $\bar{o}$ enthalten, es ist urspr. $-\vec{o} v s i$ (-vsi aus - $v, a-s, a-j, a)$ ( kann weder -övesi noch -ovesi noch -ovsi sein).

Das gesetz über die dehnung des svaritierten vocals erleidet eine ausnahme, die oben s. 498 noch nicht erwähnt $\mathrm{zu}$ werden brauchte, weil sie für die flexion des nomens nicht in betracht kommt. Es gab ausser dem normalen hochton, dem udātta, einen noch höheren hochton, zu bezeichnen etwa durch". Derselbe hat seine stelle in der reduplicationssilbe. Wir hören einen solchen höheren hochton z. b. wenn der Franzose zur bezeichnung einer grossen finsternis redupliciert 'nui' $t$, nuit', in geringerer höhe sogar in unserm nhd., z. b. wenn wir sagen 'ánmerkungen und a"nmerkungen zu ànmerkungen'. Ein urspr. a mit dem udätta wird $e$, ein urspr. a mit diesem 'udāttatara', also $e^{\prime \prime}$, wird wenn es in der sp ̈̈teren sprache den accent behält historisch zum vocal mit noch höherem eigenton, $i$, bleibt aber $e$ wenn ihm in der späteren sprache der accent entzogen wird. Das reduplicierte wort ist ursprïnglich nicht eines, sondern es sind zwei wörter: das dem reduplicierenden mit dem höheren udätta folgende reduplicierte wort behält seinen eigenen accent. Wie es heisst es-mi, eï-mi (das -mi durch ausgleichung, denn nach dem oben vermuteten muste es bei diesen an zahl geringen verben eigentlich

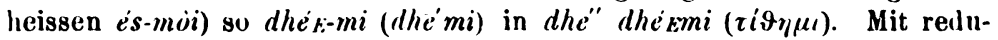
plication gebildet wurde die 1. sing. des perfects, aber wie es scheint nicht die 3. sing.: die 1. sing. hiess mit entziehung des accents gemeinindogerm. dedorca aus ailterem de" dor'cd (dar'ca $+A_{a} a$ 'ich'), die 3 sing. wahrscheinlich dirce aus älterem dor'cE ((dar'ca $+E$ 'a 'er'), beide formen urspr. mit selistiandigem svarita.

Eiu auf den hïheren udātta nun folgender selbständiger svarita bewirkt nicht die dehnung des vocals in offiner silbe vor doppeltem anulatta. Von lhara. para miiste das svaritapriisens lauten (bha'ra-, pa $\left.r a_{-}\right)^{*} b h 0^{\prime} r-m i,{ }^{*} p o ́ r-m i$, aber mit reduplication heisst es ble" bhor-mi, pe" pormi, sanskr. bibharmi, piparmi got. fara. Daher jöumi, stóumi, sanskr. ja'umi, stäumi, aber je" joùmi, zhe" zhoùmi, sanskr. jujó'mi, zuho'mi. In perfect also 3. sing. bhi'rE, aber 1. sing. bhe" bhorA. Unser perfect got. forr, môl zu fara, mala braucht keine analogiebildnng 
zu sein, sondern kann die urspriingliche 3. sing. sein: die 1. sing. war weil dem präsens zu ähnlich nicht geeignet.

Wir müssen uns dariiber entscheiden, ob wir den namen 'ablatut' künftig für den wechsel zwischen udatta- und svaritaform, $c: o$, präsens und perfect, oder für die reducierung in untoniger silbe, also für den wechsel $e:-$ und $o:--$ brauchen wollen. Von der udättaform und svaritaform, späteren $e$-form und $o$-form, ist nur zu sagen, dass sie von der ältesten zeit an neben einander bestehen: ein übergang von jener zu dieser findet soweit uns bekannt ist iberhaupt nicht statt. Präsens und perfect sind von anfang an, so weit wir sehen, von einander geschieden, als zwei getrennte verben, während der aorist zum präsens gehört. Wir sehen wohl, dass die sprache sich der neben einander bestehenden formen bedient, den unterschied des unvollendeten und des vollendeten, des imperfects und des perfects, auszudriicken, aber eine regel, wann oder warum ein wort den udatta oder den svarita trägt, ist nicht ersichtlich. Den gegensatz der svaritaform zur ulattaform könnten wir nur etwa 'tonwechsel' nennen: der name 'ablaut', von diesem gegensatz gebraucht, würde nur etwa das secundäre moment bezeichnen, dass die svaritaform kürzer ist. Sehr geeignet ist dagegen der name 'ablaut' die reducierung der tonlosen silbe und also den gegensatz der reducierten silbe zur 'guna-silbe' zu bezeichnen. Von der guna-silbe (diesen begriff richtig gefiasst) zur reducierten findet ein wirklicher übergang statt, und die reducierung ist ein sicherer sprachgeschichtlicher vorgang. Ihre ursache ist eine accentverschiebung in der richtung von der anfangssilbe fort nach dem ende hin, deren grund wenn auch nicht in jedem einzelnen falle, so doch im allgemeinen deutlich erkennbar ist. Das indogerm. wort in seiner normalstellung, unabhïngig, ist im allgemeinen auf der ersten silbe, der wurzelsilbe, betont, die verschiebung des accents nach dem ende hin wird durch das verhältnis der abhängigkeit zu einem andern worte bewirkt.

Wir können uns jetzt wider dem speciell germanischen zuwenden. Welches ursprungs sind die formen des nom. sing. der $n$-stïmme in den verschieden germanischen dialecton? Osthoff erklärt die ostgerm. endung des nom. sing. des masculius als eine analogiebildung von der art, die wenn iryend eine das epitheton 'falsche' analogic verdienen würde, die westgerm. endung des nom. sing. des feminins und neutrums aus germ. $-\bar{e} n$, das aus $-\bar{o} n$ durch die wirkung eines vorhergehenden $j$ entstanden sein soll. Wäre diese erklärung mittels des $j$ auch auf die ostyerm. endung des masculins angewant worden, dann würde die sache ein besseres aussehen haben (vgl. Paul, Btr. VI, 212). Aber es ist höchst zweifelhaft, ob 
überhaupt im germanischen ein $j$ ein folgendes $\bar{o}$ in $\bar{e}$ wandelt, und ich für meinen teil versage diesem lautgesetz den glauben. Das einzige beispiel, in dem das lautgesetz sich rein durchgreführt zeigen würde, got. jêr $=$ griech. čjos, kann das allergewöhnlichste ding sein, das uns oben zu wiederholten malen begregnet ist, das hoclitonwort neben dem tieftonwort (s. o.). Ein 'lautgesetz', dass sich nirgends rein durchgeführt zeigt, das in dem einen teil der fälle, in denen es eingetreten sein sollte, durch eine ausgleichung wider aufgehoben, in dem andern durch die entgegengesetzte ausgleichung über sämtliche fälle, wo es berechtigt und nicht berechtigt ist, ausgedehnt sein soll, braucht man nicht anzuerkennen.

Würden wir im nom. sing. der femininen $\bar{a}$-declination in germanischen dialecten mit sicherheit die endung urspr. und germ. $-\bar{a}$ neben $-\bar{o}$ vorfinden, was für das ahd. as. $-a$ altfries. $e$ gegenüber dem altengl. $-u$ altnord. ${ }^{*}-u$ der fall sein würde, wenn nicht die möglichkeit und (wegen diu, siu etc.) grössere wahrscheinlichkeit der verwendung der accusativform als nom. vorhanden wäre (Paul, Germ. XX, 105) dann würde ich nicht zu irgend einem andern kunstmittel greifen, sondern erklären das ae. $-u$ an. ${ }^{*} u$ und wahrscheinlich auch das got. $-a$ sei die ursprüngliche endung $-\bar{o},=$ umbriseh-oskisch -0 , das ahd. as. $-a$ altfries. $-e$ und vielleicht das got. $-a$ das jüngere aus den obliquen casus eingedrungene $-\bar{a}=$ griech. $\eta$. Ebenso wenn wir in den endungen des nom. sing. der $n$-declination neben dem vocal $\bar{o}$ den vocal $\ddot{e}$ vortinden, ist es mir nicht zweifelhaft, dass nirgends anders anzuknüpfen ist, als an das in den letzten abschnitten behandelte $\bar{e}$, das uns andere indogerm. dialecte im nom. sing. der $u$-stïmme zeigen. Wir betrachten die drei rencra nach cinauder.

1. Das masc. Gotisches auslautendes $-a$ kann germ. $-\bar{a}-\bar{e}$ oder $\bar{o}$ sein. Wenn man das gotische $-a$ in hana, auhsa nicht schon längst in bestimmter weise erklärt hat, so ist der grund nur der, dass man das gotische nicht vom nordischen trennen wollte. Jas gotische stimmt zwar mit dem nordischen in einer grösseren zahl von fällen überein, als mit einem andern germanischen dialecte, es braucht aber darum durchaus nicht, so weit die lautliche möglichkeit besteht, in allen fällen zum nordischen zu stimmen. Die einfachste erklärung des gotischen 
$-a$ ist zugleich die wahrscheinlichste: das $-a$ ist das ursprïngliche $-\overline{0},=$ lit. $-\imath$ latein. $-o$ sanskr. $-\bar{a}$. Gotisch hana hamins ist $-\bar{o}$-enos. Wahrscheinlich ist dies schon wegen der nahen berührung des gotischen in seinen ursprünglichen sitzen mit dem litanischen. So gut wie gewiss wird es durch die gestalt der endung des acc. sing. und des nom. plur. got. -an (aus -on-om, = griech. -ov $\alpha$ ) -ans (= griech. -oves): wäre das -a -ins ein $-\vec{e}^{\prime}-\dot{e} n o s$ dann würde dazu ein acc. $-i n=$ sanskr. -inam

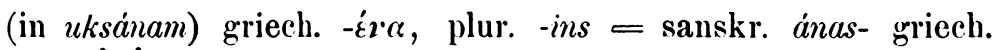

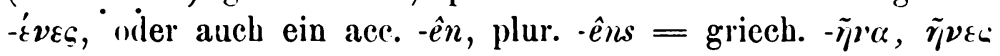
gehïren.

Das westgermanische abd. as. -o afr. ae. - $a$ kann nur germ. $-\bar{\partial} n$ aus $-\bar{o} n$ sein $=$ griech. $-\omega v$ slav. $\bar{y}$. Das ahd. hano hanin (henin) ist $-\bar{o} n \cdot e n o s=$ slav. $(k \bar{a} m) \bar{y}$-ene. Das altniederfränk. -o gen. dat. -in (=-en im Cott. des Heliand, Beitr. IV, 359) ace. -on non. plur. -on ist -ōn -enos acc. -ōnom, pl. -ōnes (im dativ neben -in aus -eni schon -on aus -ōni). Im altsächsischen ist als älteste flexion noch erkennbar -o gen. dat. -an (s. Paul, Beitr. IV, 360) ace. $-o n$, plur. $-o n$, d. i. $-\bar{o} n$ -onos -oni -ōnom, pl. -ōnes = griech. -ovv -ovos. Das -an dringt auch in den acc. sing., nicht in den plur. Später dringt -un aus dem plur. des neutr. und des fem. an die stelle des -on des plur. masc., und -un aus dem sing. des fem. in den sing. des masc. und neutr., und wir finden daher in dem uns vorliegenden texte ein constantes schwanken in masc. zwischen -on und -un. Das altengl. $-a$, $-a n$ altfries. $-a,-a$ ist $-\bar{n} n,-\bar{o} n o s$

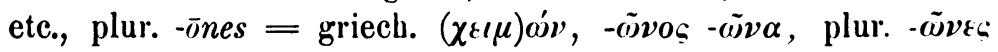
lat. gen. -ĩnis etc. Im hochdeutschen ist zum nom. -o gen. dat. -in die ältere endung des acc. sing., nom. acc. plur. -ôn (nicht -on), also -önom, plur. -ones. Daun dringt aber, im oberdeutschen frühe allgemein, im fränkischen vereinzelt, -un aus dem plur. neutr. in den plur. masc. und aus diesem auch in den acc. sing. (dieses -un wird bei Notker -en). In dem manche berubrung mit dem gotischen zeigenden bairischen finden wir noch eine dritte endung, -an, im ace. sing., nom. acc. plur., aus -on-om, -ones. ${ }^{1}$ )

1) Ein urspriingliches -incez des nom. plur. kann ahd. nur -ôn werden, ein -èce wäre ahd. -in, ein -nez (in uhsıez) wäre ahd. -an : 
Das altnord. $-i$ in hani kann nur germ. $-\bar{e}$ sein, es entspricht also dem sanskr. $-\bar{a}^{\prime}$ in $u k s \bar{a}^{\prime}$. ' Zu einem nominativ $-\vec{e}$ kann nur ein gen. -énos (nach $2 \dot{b}$, oben s. 529) oder - $\vec{e} n o s$ (nach 2 c) gehören. Das altnord. hana des gen. dat. acc. sing. ist also ein $-\vec{e} n o s,-\vec{e} n i, \vec{e} n o m=$ griech. $(\lambda \varepsilon \iota \chi) \tilde{j} v o s-\tilde{\eta} v \iota$ (das $\bar{e}$ ist genau so behandelt wie im worte altnord. máni, nur, weil

dies bezweifelt niemand. Ein -onez (got. -ans) muss ahd. -an werden. Paul, Beitr. IV, 360, VI, 223 hält zwar das ahd. -on, -un des acc. sing., nom. acc. plur. für altes -on, $=$ got. - an, aber in allen sichern fällen wird -on- (got. - an-) auslautend ahd. -an. So wird es ausnahmslos -an, niemals -on, in der unflectierten form des part. pass., und im nom. acc. sing. der substantive, die ursprünglich part. pass. waren. Zahlreiche beispiele s. bei Paul selbst, Beitr. VI, 239-242. Paul identificiert an dieser stelle das ae. - en auch ohne vorhergehenden umlaut mit ahd. -in dem umlaut voraufgeht, und trennt dieses ae. -en von ahd. -an. Dies ist absolut unmüglich. Got. -an- ahd. as. $-a n$ ist $=$ ae. -en ohne vorhergehenden umlaut, ae. Wôden, open, morzen, azen, dieses ist altes -on-. Dagegen got. -in- ahd. as. - en, -in ist $=$ ae. -en mit vorhergehendem umlaut, $h \hat{a} \partial e n, \hat{a} z e n$, myrzen = got. maurgins, germanisch -en-. Ae. -on endlich, das Paul mit ahd. as. -an identificiert, ist germ. -un aus $-n,=$ ahd. -un, ae. eoton wie ahd. Ermun- (ahd. Erman- ist ae. eormen-, ahd. irmin- ae.yrmen-). Die meisten dieser wörter sind ursprüngliche neutra auf - $\dot{\prime} n$, gen. -ini-s, (s. s.519), die dann in die o-decl. und z. t. ins masc. genus übertraten, also maurgin-s ist ein neutrum "mérzhon, gen. ${ }^{*}$ mrzhinos. Airmana- ist adj. *irA.mon-, gen. rAmenos (s. ZDA. XXIII, 3). Die dritte stufe auf -un ahd. -un ae. -on stammit aus dem ersten gliede von compositen, also ermun- in lat. acc. Hermun-duros ist ${ }^{*} r m n$ - mit umwandlung des ur der ersten silbe zu or nach der form der starken casus. Ganz ebenso steht es mit den von Paul VI, 244 behandelten wörtern auf $\iota$ und $r$, ahd. uodal wäre urspr. "átor, gen. *ateris, ahd. fezzil "pedor 'p déros. Das nordische -an--al-ist nicht mit Paul dem ahd. -an -al gleichzusetzen, es ist vielmehr das -ōn-, - $\bar{r} r$ - der ursprünglich dreisilbigen starken casus, an. ojal stammt also aus dem plur. " $\bar{a}^{\prime} t \bar{o}^{\prime} r A$.

Also ein ultes -on- kann ahd. im auslaut nur -an werden. Was ist daun aber die endung des acc. sing., nom. plur. -on in den ältesten oberdeutschen und den bauptsächlichsten fränkischen quellen? Ueberall wo dies -on constant und von -un des plur. neutr. streng geschieden ist kann es gar nichts anderes sein als -ôn. -on mit kurzem vocal, ausser wo -on fürs - un eintritt, ist fürs ahd. eine ganz unmögliche endung. Ein solches.-on giebt es sonst gar nicht. Zu schreiben ist also nom. plur. Frankîn u. s. w. Das $\hat{o}$ ist genau dasselbe wie im gen. plur. Frankìno. Der gen. Frankôno hat sein $\hat{o}$ nirgends anderswoher als aus dem nom., so sicher wie das $\bar{o}$ in gen. Scipiōnum nicht das heribergenommene $\bar{o}$ von orctionum, sondern das o des now. Scipiones ist. Das ahd. -ìn des 
nicht in der tonsilbe, verkiirtt). Das nordische hat neben diesem $-\vec{e} n o s,-\vec{e}^{\prime}$ nom ohne zweifel ursprünglich auch ein -ōnom, wozu gen. $-\bar{n} n o s$ besessen: auch dieses muste im gen. dat. acc. sing. an. - $a$ werden, es kann also zu einer zeit, wo diese flexion mit $\bar{o}$ und die mit $\bar{e}$ überall ausser in nom. sing. zusammengefallen waren, von deu beiden singularnominativen der eine aufgegeben sein. Das $\bar{e}$ hat sich, wie wir oben sehen, vornehm-

plurals reimt auf das - $\hat{o} n$ des infinitivs. Die schreibung mit kurzem $o$ ist übrig geblieben als ausläufer jener zeit, welcher Braune ein ziel setzte, wo man im ahd. mechanisch einen circumflex setzte wo in gotischen ein langer vocal stand, dagegen denjenigen vocal kurz sein liess, der im gotischen kurz war. Dass Braune dem nom. plur. Frankôn die kiirze liess, riihrt daher, dass Notker für dieses $o$ nicht die lïnge bezeugt, und dies daher, dass Notkers dialect dieses -in ïberhaupt nicht mehr hatte, sondern las aus dem plur. des neutrums herübergenommene -un voraussetzt. - Otfrid hat das -un nur im adj. in nom. acc. plur., nicht im acc. sing. Hier kann das -un gar nichts anderes sein als aus dem neutrum entlehnt. Die entlehnung aus dem neurrum lag nahe, da die flexionsendungen des masc. und neutr. im ganzen iibereinstimmen, und im adjectiv am nächsten. Ueberall nun, wo im nom. acc. plur. des masc. ausschliesslich -un herscht und iberall wo mit -on schwankendes -un vorliegt (8. Braune, Beitr. 11, 150, Paul, IV, 362, Kögel, ker. gl. 166 f.) ist dies -un aus dem neutrum herübergenommen. Es ist dies im gesamten oberdeutschen der fall schon in den älteren quellen. Dass das -un hier ausser dem -ôn des nom. acc. plur. auch das -ìn des acc. sing. verdrängt (Notker hat $-e n$ in beiden fällen), und nicht die vocalliange des accusativs im singular mit sonst kurzvocalischen endungen isoliert stehen bleibt, ist nicht wunderbar.

Dass in den verschiedenen germanischen dialecten die endungen des nom. plur. masc. -onez und -önez nebeneinander bestanden ist deutlich an den überlieferten gerınanischen völkernamen nach der $n$-declination zu sehen da wo die quantität des o bezeichnet wird, also bei den griechischen schriftstellern. Y'tolemäus (II, 11, III, 5) hat in Oberidelits'll-

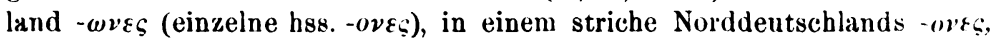

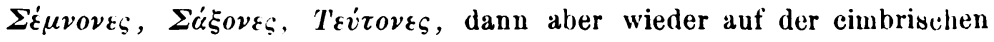

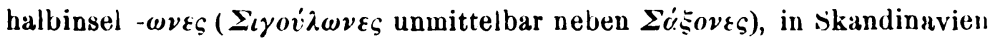

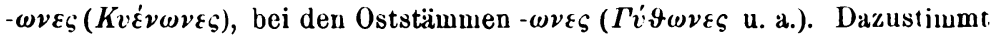
dass bei Plutarch im Marius (kap. 15 fi.) constant neben einander stehen

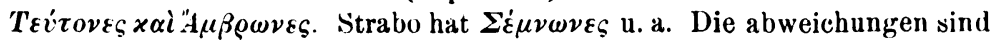
natiirlich, da man ja die namen nicht immer von den völkern selbst, sondern auch von den nachbarn hörte. Im einzelnen ist auf die schreibung mit $o$ oder $\omega$ nicht viel gewicht zu legen, dass aber-onez und $\overrightarrow{o n e} z$ in den germ. dialecten nebeneinander bestanden ist unzweifelhaft. -ön-im acc. sing. zcigt lie flexion

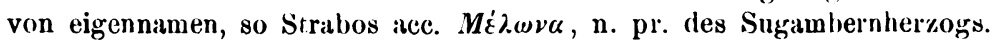


lich in solchen wörtern eingrestellt, welche den accent der obliquen casus verallgemeinerten. Dazu stimmt, dass das nordische wort dän. schwed. hare hase ein älte:es oxytouon ist (= griech. $-i / v$, joos), das deutsche haso und fries. hasa ein

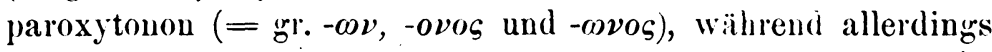
das enrlische hara im aceent zum nordischen stimmt (=- -ón, -(ijvos). Das wort bätte nuit beiisehaltung des alten ablauts zu lauten got. *hòsa, *hazins: möglich dass das isl. héri die vocallänge der starkeu casus festgehalten hat (als compromiss aus *hori, gen. *hera? 1)). - In plural musten -ēnez und -ōnez zusammentallen, der plural an. hanar, $-a$ etc. aber ist in jüngerer zeit nach dagar, -a etc. gebildet, gumnar natiirlich noch jünger.

2. Das neutrum. Die ursprüngliche endung des nom. sing. war bei alten properispomenen - $n$ (nōmn name, lat. -en sanskr. - $a$ griech. $-\alpha$ ), wozu der gen. -nos, bei alten paroxytonen -on, wie *érdon herz (s. o.), wozu der gen. -énos. In allen indogerm. dialecten hat zwischen den beiden endungen ein ausgleich stattgefunden.

Das -on der ursprïnglich zweisilbigen starken casus, des nom. acc. sing., ist im germanischen dem $-\bar{o} n$ - der ursprünglich drcisilbigen starken, des nom. acc. plur., gewichen, das germanische setzt also für den nom. acc. sing. der paroxytona ein $-\bar{o} n$, germ. $-\bar{o} n$, voraus. Die hauptsächlichsten wörter dieser endung waren germ. hértōn herz, sết mōn same, séimōn schnur. Die cndung des nom. acc. plur. dieser wörter war urspr. -ōna. Diese endung wird vorausgesetzt durch den ahd. und as. gen. plur., ahd. herzòno.

Die hauptsächlichsten wörter auf $-n$, germ. - - nn, plur. ursprr. -na, waren die wörter 'auge', 'ohr' und 'name'.

('ot. hairtô, -ins, -in ist -ön, -enos, -eni. Diesclbe flexion hahen die wörter auf -n angenommen, got. namô, vatô, ausô, angò. Der plur. got. hairlòna, -anê ist -önō nach der o-decl., gen. $-o m-\vec{e} m$ (s. s. 1\$9). Im plural haben nur 'auge' und 'ohr' diese 'flexion faible' (oder 'Lochtonflexion') angenommen, namna, vatna bewahren die syncopierte gestalt der 'flexion forte' ('tieftonflexion').

1) Ebenso an. fani alus * föri gen. fana? (ahd. màyo mhd. màge mohn, wenn dies irgendwo sicher latugen vocal hat, durch dieselhe ausgleichung? s. s. 517.) 
Der nordische nom. acc. gen. dat. sing. hiarta, sima ist jüngeres $-\bar{o} n,-\bar{o} n o s,-\bar{o} n i$. Dieselbe gestalt haben auga und eyra angenommen. Es kann aber mit diesem $-\bar{o} n,-\bar{o} n o s$ in dem nordischen $-a$ ein $-\bar{e} n-\bar{e} n o s$ (nach 2 c, s. 531) zusammengefallen sein. An. sima ist germ. séimōn, es könnte aber an. eyra ein vornord. auz $\vec{e} n$ sein gegenluber dem got. ausô aus vorgotischem $a ́ u s \bar{o} n$. Ueber den nordischen nom. acc. plur. s. u.

Im westgermanischen haben wir im nom. sing. teils das $-\bar{o} n$, teils ein nach dem -én- der obliquen casus an die stelle des $-\bar{o} n$ getretenes $-\vec{e}^{\prime} n$, wie wir es im slavischen, sēmę, und im preussischen, sēmen, widerfinden (s. Leskien, Decl. s. 63 f.). Der $o$-laut blieb in germ. sêe $m \bar{o}^{n}$ same, séimo $n$ schnur, ahd. as, -o afi. ae. $-a$, afr. ae sima schnur. Diesen wörtern schloss sich das ursprünglich der 'flexion forte' angehörige ahd. as. namo afr. ae. noma name an. Diese wörter hatten nunmehr vollständig die flexion des masculins (bis auf den acc. sing.), denn der plural fiel in der gestalt $-\bar{o} n$ aus $-\bar{o} n a$ mit $-\bar{o} n$ aus $-\bar{o} n e z$ zusammen. Sie sind daher im westgerm. auch ins masculine genus übergetreten. Den $\bar{e}$-laut nahm dagegen das wort 'herz' an (also hert $\vec{e} n$ nach dem gen. -énoz mit der stammsilbe der starken aber dem accent der schwachen casus wie got. fairzna?). Ahd. herza -in altndfränk. herta, -in ist ein -ēn -enos -eni. Im friesischen und englischen dagegen ist das $\bar{e}$ auch in die obliquen casus gedrungen, ae. $-e$, -an altfries. $-e,-a$ ist ein $-\bar{e} n-\bar{e} n o s-\bar{e} n i$, ebenso finden wir im altsächs. im dat. sing. hertan, das -êni sein kann, freilich auch aus dem -an des masc. herlbergenommen sein kann (wie das -on in herton). Im plural blieb zunächst wie im masc. der $o$-laut gewahrt: das altsïchs. herıon gen. -ono ist $\bar{o} n \bar{o}$, gen. $\bar{o} n \bar{o} m$, ebenso setzt der ahd. gen. plur. herzôno einen früheren nom. herzôn voraus. Eindringen des $\bar{e}$ in den nom. acc. plur. sehen wir im niederfränk. (auch im Heliand M. sich findenden) plur. hertan (aus $-\bar{e} n \bar{o}$ ) neben -on des masculins. Im englisch-friesischen ist das wort 'herz' feminin geworden, warum wol? Wahrscheinlich deshalb weil es ursprünglich von allen neutren allein im sing. und plur. genau dieselbe flexion hatte, wie das fem., z. b. wice woche, während die wörter 'auge' und 'ohr' noch der 'flexion forte' folgten.

Die wörter 'auge' und 'ohr' hatten im ursprünglichen 
germanischen im nom. acc. sing. die endung -un aus -n, im plural die endung -na. Den plural oder dual in der gestalt $-n \bar{o}$, germ. augnō, auzno nach der o-decl., hat wie wir oben sahen, das schwedisch-dänische, augno ebenso das friesische bewahrt. Im niederfränkischen finden wir nun den plural ougun (von hertan und von dem -on des masc. geschieden), im hochdeutschen ougun, ôrun (und danach herzun, ursprïnglich geschieden von der endung des masculins), im altnordischen augu, eyru (welche endung sich dann aber auch den übrigen neutren mitteilt). Lautlich konnte dies -un, $-u$ nicht aus $-n a$, sondern nur aus -na entstehen, aber von augna ist nicht zu einem augna zu gelangen. Dieser plural ougun an. augu wird also nichts anderes sein als ein plural nach dem vorbild von ahd. wort, pl. wort, an or $\delta$, pl. or $\delta$ aus einem älteren singular ahd. anfrk. *ougun an. *augu gebildet.1) Dieser fruhere singular hatte die ihm gebuhrende endung, vgl. ahd. sibun, niun, as. nigun, an. niu, tiu. Der plur. ae. éazan, éaran afr. âra ist in jüngerer zeit dem masc. und fem. nachgebildet.

3. Das feminin hatte ursprüglich genau so wie das masc. die endung nom. sing. $-\bar{o}$, gen. - énos, acc. $-\bar{o} n m$, plur. -oness. Die lehre, dass es urspringlich keine feminina auf $-n$ gegeben habe, lehrt etwas unmögliches, denn die consequenz wäre, dass die sprache von allem anfang an ein genus masc. und fem. unterschieden habe. Die verschiedenen suffixe $-A,-i$, $-u,-r,-n$ etc. dienen urspriinglich absolut nicht zur unterscheidung eines grammatischen genus nach unserem begriffe: es rab ursprünglich suffixlose, wurzel-nomina auf -0 , nom. sing. $-o-s$, als feminina so gut wie als masculina, sieh das griech. und lat. das hierin nur älter, nicht jünger scin kann als die iibrigen dialecte $\left.{ }^{2}\right)$, es gal) masculina auf $-A$ so gut wie feminina, und die ältesten wörter der sprache, wie $z$. b. die verwantschaftswörter, zeigen von einer unterscheidung des genus keine spur. Erst in einer jungeren zeit hat die sprache sich der verschiedenen stammauslaute $-e-(=-o-),-\bar{a}-,-e n-$, als eines willkommenen mittels bedient, um ein grammatisches genus zu

1) Ebenso haben wir im obd. auch herza und danach auga als plur.

") Für adjectivisch gebrauchte composita von $o$-stïmmen kann das einzig urspriingliche sein masc. und fem. $-0-s, n$ tr. $-0-m$, wie griech. fem.

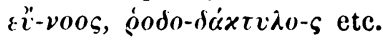


unterscheiden. Wenn es in indoiranisehen keine (oder nur wenige) feminina auf $-n$ giebt, so sind sie nach dem grleichen nominativausgange $-\bar{o}(-o A)$, indoiran. $-\bar{a}$, in die declination der feminine auf $-A$ übergetreten. Im germanischen, wo die feminine auf $-A$ ihren alten nominativausgang $-\bar{\jmath}$ am längsten behielten, sind umgekehrt nach dem gleichen ursprïnglichen nominativausgang zahlreiche feminina auf $-A$ in die $n$-declination ïbergetreten, z. b. got. kvinô etc. Wir haben in diesem übertritt einen neuen beweis für den ursprïnglichen gemeingermanischen nominativ auf $-\bar{o}$ der $A$-stämme, denn ohne diesen nominativ wäre der übertritt unmöglich gewesen. - Ein zweifellos altes feminin auf $-\bar{o}$-énòs ist \%. b. das lateinische virgo, -inis, das zum griech. $\pi \alpha \rho \vartheta \varepsilon \dot{v o s}$ gehalten (mit dem von Joh. Schmilt als gesetzlich erwiesenen ibhergang des $k$-lautes ror $e, i$ in den $t$-laut). uns ein altes ghu'rghui, gen. ghurghuinòs zeigt. ${ }^{1}$ )

Im germanischen ist, natürlich erst nachdem wörter wie gu'no' weib in diese n-declination eingetreten waren, das $"$ aus den ibbrigen casus auch in den nominativ gedrungen. Im gotischen und nordischen haben wir im nom. sing. die endung $-\bar{o} n$, germ. kuénön, gotot. kvinò an. koma. Im gotischen masce. -a aus $-\bar{o}$, fem. $-\hat{o}$ aus $-\bar{n} n$ haben wir also das umgekehrte verhältnis wie im mriechischen, wo zum masculin auf - $-\omega v$ das feminin (neben der gestalt auf $-\omega v$, čndón, cix(i)v -óvos f.) die ältere gestalt -(1) wahrt, $a j y d \omega^{\prime}$, rix(i) (das dann in die $i$-decl. eintritt,

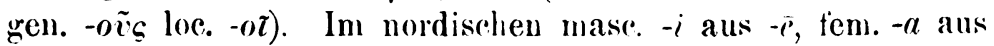
$-\bar{m} n$ dagegren besteht das umgekehrte verhältnis wie im lateinischen, das zum $e$-laut im nominativ das $-n$ treten lässt, liēn, vom o-laut es aber fernhält, homo, virgo. ${ }^{2}$ ) Das gotische hat beim feminin nicht wie beim masc. und neutr. die ursprüngliche abstufende flexion, gen. -ins aus -inos bewabrt, sundern den

1) In inlaut lat. g fiir $g u$ wie in urgeo, im anlaut $g v$, woraus $v$, wie das $g, b$ in gilvus. barba, und auch sonst haiufig media fiir urspr. aspirata vor folgendem $r$. Wureiverwant kann sein das niederdentsche gör n. kleines mädchen, das got. *gaurvi wäre (vgl. mit demselben laute des $\ddot{o}$ nnd. $m \ddot{r}=$ ahd. muruwi), und ne. girl (iilter auch 'knabe'), got. *gaurvilio. (Lit. merga mä̈lchen aus $g^{u-}$ wie ma'mas haus aus damas?).

2) Dass im nordischen ursprünglich $-i^{n}$ auch im masc., - $e$ auch im fem. galt, sehen wir an einzelnen die jiingere regel durchbrechendeu eigennamen, masc. Sturla, fem. Skaji. 
langen $\bar{o}$-laut der starken casus (nach 1, c, s.527) in die gesamite flexion dringen lassen: - $\hat{o}$-ôns ete. aus $-\bar{o} n$, gen. $-\bar{o} n o s$, plur. -onnes. Von der flexion des feminins in nordischen unten.

Las westgermanische hiilt es wie das griechische (der meisten dialecte), indem es in allen fällen das $-n$ in den nom. sing. treten lïsst. Das westgermanische hat aber, während es im mase. der endung des nominativs -ion zum gen. -enos den $o$-laut wahrte, im feminin den e-laut der obliquen casus in den nominativ dringen lassen (nach 2 b, s. 529): ursprünglich $-\vec{\sigma}$, gnen. -énos, dann $-\bar{o}^{\prime} n$ gen. -énos, endlich $-\bar{e}^{\prime} n$ iren. -énos. $\left.{ }^{1}\right)$ Also westgrerm. vik $\vec{e} n$ woche (das $-\bar{e} n$ wird $-\bar{a} n,-\bar{a}$, endlich $-a$ ), abd. wecha ae. wice. Neben diesem oxytonierten $-\vec{e}^{\prime} n$ wird aber im westgermanischen ursprïnglich auch ein barytoniertes $-\bar{o} n$ im fem. bestanden haben, z. b. in *kvénōn, das dem gen. plur. ahd. -ono as. -ono seinen $\bar{o}$-laut hinterlassen hat (der gen. -ovos noch in rreck. kòpan-(bandi)), in nom. sing. aber später dem - $\vec{e} n$ gewichen ist (dieses $-\bar{o} n$ wird durch lehnwörter wie $s \bar{a} p o$ seife vorausgesetzt). Das englisch firiesische lässt das $\bar{e}$ des nominativs in die gesamte flexion eindringen, ae. wice, gren. etc. wican, a fries. wike, wika aus $-\bar{e} \mathfrak{n}$,

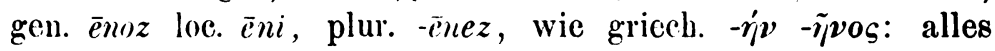
ausser dem nom. sing. kamn aber auch - onnoz, pl. -ōnez sein, es wird also nachdem beide flexionen bis auf den nom. sing. cinander gleichgeworden waren von den zwei nominativen der cine aufrgegeben sein.

Noch ein wort über das $\bar{u}$ in gen. lat. acc. sing., nom. acc. plur. abd. zungîn altsächs. tungun, altnord. gen. dat. acc. sg. tungu nom. acc. plur. -ur. Ich weiss von keinem, der dies $u$ für etwas anderes hält als ursprüngliches $\bar{o}$, ich weiss aber auch nicht wie viele sich bei diesem postulat beruhigt haben, orler wio sic das lautgeset $\%$ formuliert haben, nach welchem

1) Möglich dass das westgernanische beim comparativ und superlativ den e-laut der obliquen casus in den uous. sing. des masculins hat treten lassen, während hier das feminin urspriinglich gemeingermanisch ¿uf - - ausging: as. Heliand masc. betera, bezta (s. Paul, Beitr. IV, $346 \mathrm{f}$, frirs obd. s. Kögel, Keron. gloss. s. 165 f.) aus batizën, gen. batizenoz, noben dem fem. batizīn (das urspriingliche war, wenn wir das wort mit unverschobenen lauten schon als $n$-stamm flectieren dürfen, $b h \bar{a}^{\prime} d i s \vec{o}$, gen. bhadisénos, s. 0. 8. 506). Vielleicht aber ist hier das -a des nom. sing. wasc. nur jüngere formübertragung aus dem neutrum und demnächst auch aus dem fem., ebenso wie das -un für -on der übrigen casus. 
sie hier das auftreten des $\bar{u}$ für $\bar{o}$ erklären. Paul vermutet Beitr. VI, 223, dass das $\bar{u}$ aus $\bar{o}$ als urgermanisches in acc. sing., nom. acc. plur., oder auch nur im acc. sing. und plur. seine stelle gehabt babe, und dass dann im got. und ae. das $\bar{o}$ der übrigen casus das $\bar{u}$ verdrängt, im hochd. und nord. das $\bar{u}$ auf kosten des $\bar{o}$ sich ausgebreitet habe. Wir haben genug lange $\bar{o}$ im laufe unserer darstellung angetroffen, und genug andere kommen uns sofort in den sinn, wenn wir die langen $\bar{o}$ in nebensilben mustern, von einem ibergang eines langen $\bar{o}$ im älteren germanischen in $\bar{u}$ in nebensilben vor folgenden consonanten, sei es auch nur vor $n$, unter irgend welchen bedingungen, ist nirgends etwas $\mathrm{zu}$ bemerken.

Es ist bekannt welches ursprungs die in derselben weise wie $u g g \hat{o}$, gen. -ôns, flectierenden feminina auf $-\hat{\imath}$ sind, got. managei, gen. -eins etc., deren flexion germanisch anzusetzen ist nom. $\bar{\imath} n$, gen. $\bar{\imath} n o z$, plur. $\bar{n} e z$. Es sind alte stämme auf $-\bar{\imath}$, älter $i A$, die, wie sie im sanskrit den gen. plur. -innam bilden, so im germanischen völlig in die $n$-flexion eingetreten sind. Diesen $\bar{i}$-stämmen sind nun aber in den verwanten sprachen stïmme auf $-\bar{u}$, älter $-u A$, völlig parallel. Solche sind svecr $\bar{u}$ schwiegermutter, älter svecrud (sanskr. cvacrǘ-slav. svekrÿ), $d n \bar{z} h \bar{u}$ zunge (das slavische und das preussiche setzen ein gemeinsames ${ }^{*} \eta z \bar{u}$ aus $d n z \bar{u}$ voraus (s. Bezz. Beitr. III, $133 \mathrm{ff}$.), las im slavischen durch -ko- weitergebildet ist, jezzy-kŭ) aus dnghuA (lat. dinguı, lingua). Ihre ursprüngliche flexion war gen -uAos, woraus -ūos (=-uvos), slav. gen. svekrŭve, sanskr. gen. cvacrǘas (-úvas, -vàs). Der gen. plur. hat im sanskr. die gestalt $-\bar{u} n \bar{a} n_{i}$, wie olben bei den $\bar{\imath}$-stämmen -ìnām, bei den $\bar{a}$-stämmen - $\bar{a} n \bar{a} m$. Was aus diesen femininen $\bar{u}$-stämmen im germanischen geworden ist liegt nun auf der hand: sie sind in germanischen ebenso wie zahlreiche weibliche $\bar{o}$ - $(\bar{a}-)$ stämme und wie jene $\bar{\imath}$-stämme völlig in die $n$-flexion eingetreten, wie in sanskrit im gen. plur. (und wie im sanskrit die neutra auf $-i$ und $-u$ allmählig völlig $n$-flexion annehmen, gen. -inas, -unas etc.). Das gemeingermanische besass also eine der flexion ${ }_{-i} n$, gen. -ìnoz vōllig parallele flexion svehrün, tungūin, gen. $-\bar{u} n o z$, plur. -ünez. Im gotischen sind diese wörter völlig in die klasse der wörter wie kvinô, vikô eingetreten, svaihrô, tuggô. Wäre das alte $-u A, \bar{u}$ nicht in dem $-\hat{o}$ zu suchen, dann wirden 
wir in verlegrenheit kommen, wenn wir tiber den verbleib des $u$ nach dem palatal in tuggô $=$ lat. lingua rechenschaft $a b$ legen sollten (vgl. fidvôr). Im englisch-friesischen haben sie denselben völligen ubertritt gemacht, im nordischen und deutschen aber haben sich die wörter auf $-\bar{u}^{n}$ mit denen auf $-\bar{o}^{n}$ in der weise verschmolzen, dass jene den acc. und die obliquen casus des sing. und den nom. acc. plur., diese den nom. sing. und die obliquen casus des plurals hergaben. Dies muss geschehen sein zu einer zeit, wo im westgerm. noch (wie in (riech. masc. $\pi \lambda \varepsilon \dot{v}_{\mu} \mu \nu$ neben $\left.\pi \varepsilon v \vartheta \eta^{\prime} v\right)$ im fem. das $-\bar{o}^{n}$ neben dem $\bar{e}^{n}$ bestand. Jenes $\cdot \bar{o} n$, das im nom. sing. im westgerm. später völlig schwand, hat in ahd. zungôro as. tungono seinen gen. plur. hinterlassen (das altnord. tungna ist wie hiartna $-o n-\bar{o} m)$. Die ahd. flexion von zunga hat sich also aus folgenden grundformen zusammengesetzt:

$$
\begin{aligned}
& \text { sing. nom. }-\bar{e} n \text { gen. }-\bar{u} n o s \text { plur. nom. -iunes } \\
& \text { dat. }-\bar{u} n i \quad \text { gen. }-\bar{o} n \bar{o} m \\
& \text { ace. -ünom dat. nach der } \bar{o} \text {-decl. }
\end{aligned}
$$

Im nordischen muste, wie germ. álbīn, gen. -ìnoz etc. in allen casus des sing. an. elli wird, so tung $\bar{u} n,-\bar{u} n o z$ im ganzen sing. tungu werden. In ahd. ist als älteste flexion der wörter auf $-\bar{\imath}$ noch zu erkennen nom. sing. manegì gen. dat. acc. manegîn, wenngleich nirgends mehr so erhalten (das $-\hat{\imath}$ des nom. sing. setzt ein an die stello des $-i n$ getretenes $-i n_{z}$ voraus): diesem wïrde parallel sein nom. ${ }^{*} z u n g \hat{u}$ gen. dat. acc. zungûn. ${ }^{1}$ )

1) Es sind urspriinglich fïr das ganze indogerm. gebiet zweierlei wörter auf $-t$ und $-\bar{l}$, ïlter $-i A,-u A$, $z u$ scheiden, die freilich in allen dialecten vielfach in einander iibergreifen: die eine klasse im nom. sing. les $-s$ rntbehrend und in der flexion den femininen auf -1$)(-0 A)$ sich

\begin{tabular}{|c|c|c|c|}
\hline \multirow[t]{2}{*}{ Urspr. } & $\begin{array}{l}\mathrm{n}(\mathrm{m}) \\
-i \mathrm{~A}\end{array}$ & $\begin{array}{r}\text { acc. } \\
-i A m\end{array}$ & $\begin{array}{l}\text { gen. } \\
\text {-iAits }\end{array}$ \\
\hline & $-u A$ & $-n-4 m$ & $-u-t i s$ \\
\hline \multirow[t]{2}{*}{ sinskr. } & -1 & $-i m$ & $-i \pi s$ \\
\hline & $-\bar{u} s$ & $-\bar{n} m$ & $-M i s$ \\
\hline griech. & $-\imath \alpha$ & $-\iota \alpha \nu$ & $-\| \bar{u}$ \\
\hline \multirow[t]{2}{*}{ latein. } & $-i a$ & -iam & -iae \\
\hline & $-r e a$ & $-2 k a m$ & -uac \\
\hline
\end{tabular}
nabe anschliessend, die andere mit $-s$ im nom. sing.

I.
II.

nom. ace. gen. sing.

$-i s \quad-i m \quad-i A O S$

-ïs - lim -uAos

-is -iam -ias

$-\bar{u}^{\prime} s-u^{\prime} a m-\bar{u}^{\prime} a s$

- $\bar{v} s \quad-\bar{v} v \quad-v o s$

-is (neptis)

-us (socrus)

-io, -ion-, ital. -in- 
Die casusendungen der $n$-declination und die der von uns fruher betrachteten o-declination in den verschiedenen germ. dialecten gehen in rein lautlicher entwicklung auf die folyenden von einander abweichenden, hier in vorgermanischer lautgestalt angesetzten formen zurück.

\begin{tabular}{|c|c|c|c|c|c|c|}
\hline & & N-decli & on. & gular. & & \\
\hline & & Urspr. & got. & an. & ahd. & ae. \\
\hline Masc. & nom. & $-\overrightarrow{0}$ & $* \vec{o}$ & $*_{\bar{e}}$ & $* \overline{o n}$ & $* \bar{o} n$ \\
\hline & acc. & $-\vec{o} n-m$ & *onom & *Ẽnom & * $\bar{o} n o m$ & *onom \\
\hline & gen. & $-i m l s$ & * enos & *ènos & *enos & * $\bar{\delta} \| n s$ \\
\hline neutr. & nom. & $-n,-\dot{v} n$ & $* \overline{o n}$ & $* \bar{\delta} u$ & *in & $* \bar{e} \|$ \\
\hline & gen. & -inis, - -inns & *éness & * $\bar{o} n o s$ & *enos & *ènos \\
\hline fem. & nom. & $-\pi$ & $* \overline{0} n$ & $*$ onn & *èen & $* \bar{e} n$ \\
\hline & acc. & $-\overrightarrow{0} \| m$ & *innom & $\ldots$ & $\ldots .$. & * \\
\hline & gen. & $-\operatorname{conos}$ & ${ }^{*}$ onnos & - & - & ${ }^{*}$ ènos. \\
\hline
\end{tabular}

0-der.l. (s. 185-491). Sing.

yen. $-i s o$, got. ahrl. as. "eso, as. ac. an. *oso. $)$

lok. $-i i$, ahd. ae. an. ${ }^{*} o i$.

lat. $-e^{\prime} i$, ahd. as. " $\partial i$.

instr. $-e$ ', got. ae. ${ }^{*} \bar{e}$, ahd. as. ${ }^{*} \bar{o}$.

Plur:

nom. m. -is, got. an. "ös, ae. afr. as. "isses. $\left.{ }^{2}\right)$

ace. m. $-\vec{o} \|$, an. ${ }^{*} \ddot{z} n$, got. ${ }^{*}$ ons. $\left.{ }^{3}\right)$

, n. $-i^{\prime}$, got. an. ac. as. ${ }^{*} \bar{o}$, ahd. $\left.{ }^{*} a .^{4}\right)$

gen. $-\tilde{c}^{\prime} m$, got. " $i m$, sunst ${ }^{*} i m$.

') s. $5(11)$ anm. 2) 8. $505 .{ }^{3}$ ) 8. 488 anm. 4) s. 48.4 anm.

Bei den tiefgehenden differenzen zwischen den gemmanischen dialecten werden wir uns eine möglichst einheitliche germanische grundsprache nur dann annïhernd richtig vorstellen, wenn wir dieselbe möglichst viel von den urspring-

$\begin{array}{llllllll} & \text { noml. } & \text { acc. } & \text { gen. sing. } & \text { nom. ace. gen. sing. } \\ \text { got. } & -i & -j a & -j i s & & -e i & -e i n & -e i n s \\ \text { nord. } & -r & -i & -j a r & -i & -i & -i .\end{array}$

Unsere germanischen wörter auf $-i n-$ und $-\bar{u} n-$ sind also nicht aus der ersten, sondern aus der zweiten klasse mit uspriinglichem $-s$ im nom. sing., wie skr. svacru't-s lat. socru-s, hervorgegingen. Im nordischen ist bei den wörtern auf $-i$, wie $y l g r$, genan so wie im sanskrit bei den wörtern auf $-\bar{u}$, das $-s$ des nom. sing. in die erste klasse eingedrungen. Den iibertritt in die $n$-flexion, den wir bei den wörtern der zweiten klasse im germanischen sehen, zeigt bei den $i$-stäinmen derselben klasse auch das it:alische, acc. oskisch (tang)-innom gen. mus dat. -inei, abl. -inud umbr. -ine, nom. osk. -iuf lat. -io. 
lichen gencinsam indogermanischen gewahrt haben und mö̈lichst viel innerhalb der wenn auch in beständiger fühlung mit cinander lebenden einzelnen germanischen dialecte entstanden sein lassen. Wer die grammatik eines germanischen dialects behandeln würde, ohne über die grenzen des germanischen hinauszublicken, würde keinen boden unter den füssen haben.

KETTING ALF ALSEN, ช. APRIL Is>I.

HERMANN MÖILER.

Nachträge \%un excurs s. 492 ff. s. 496 . Die entstehung des durch den tiefton lehrt jetzt auch Fick, Gött. gel. anz. v. 7 april 1sst).

4!9) anm. 3. Das zahlwort 'vier' war vielmehr hochtonwort (zu

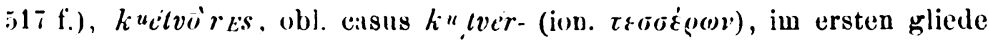
eines comp. $k u t v r-(\tau \& \tau \rho \alpha-s k r$. catur-got. fidur-), woher die tieftonflexion iin sanskr. und griech. nach s. 521.

514. Das germ. $u$ fïr $\bar{u}$ in sunus (analog das $i$ fïr $i$ in kvius) u. a. ist entstanden durch ablautwechsel $e u: u$ für $\iota u: \bar{u}$.

515. Das $-A,-i,-r$ des nom. sing. wird darin seinen grund haben, dass (wie $i, j$ neben stamm $\tau o$-) in einer urzeit das pronomen - $A a$ bei belebten, $-j a$. -ra bei unbelebten wesen für den nom. sing. des artikcls gebraucht ward neben sonst als artikel verwantem pronomen - na, -ja, -ra. (Das - A aus -Aa entbehrt des nominativ -s genau wie ó.)

521. Das lat. ete. $j$ in jecur $=$ germ. $l$ ist aus urspr. $l j$ entstanden, vgl. das $j$ aus $l j(l l)$ im schwed., französ., span.

521 f. Das wort 'eis'u' War urspr. ëisor n., gen. isénos (das germ. $z$ der obliquen (asus in ae. iren). Das -or-n, jiinger nach der o-decl. -arma-, in eisarma- (altnordhumbr. if'ern ahend u. a., ebenso wol in germ. shar-na-) ist ein seitenstick zu lat. jec-in-or-is, oder das -" hat sich grebildet wie das $-t$ in skr. jakr-t.

52.4 anm. I) its slavisch-litauische scheidet $r \cdot A, / A$ vou $r, l$ durch den aceent. s. sueben Fortunatov in slav. archiv IV, 5is ff.

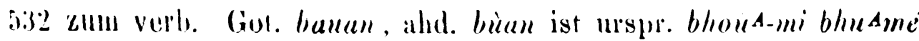
(vyl. Paul, htr. VII. 155). -- Auf ein -sii der 2. sing. (dessen stelle nach vorhergehendem é, és-sii, rïki rike-siii) weist das slav. -si (-ji). Dic huchtonwijpter der noch ungeschiedenen schwathen $e^{-}$und o-conjunation (nicht die causativen verhen, deren stammsibe tieftonig) flec-

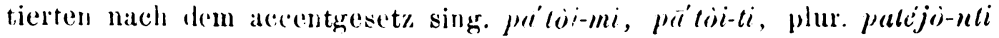
ulit: 3. singr. got. -aib ahil. -it also ans -oiti, niclit alls -ojeti). 